\title{
Topological strings on genus one fibered Calabi-Yau 3-folds and string dualities
}

\author{
Cesar Fierro Cota, ${ }^{a}$ Albrecht Klemm $^{a, b}$ and Thorsten Schimannek ${ }^{c}$ \\ ${ }^{a}$ Bethe Center for Theoretical Physics, Universität Bonn, \\ D-53115 Bonn, Germany \\ ${ }^{b}$ Hausdorff Center for Mathematics, Universität Bonn, \\ D-53115 Bonn, Germany \\ ${ }^{c}$ Faculty of Physics, University of Vienna, \\ Boltzmanngasse 5, A-1090 Vienna, Austria \\ E-mail: fierro@th.physik.uni-bonn.de, aklemm@th.physik.uni-bonn.de, \\ thorsten.schimannek@univie.ac.at
}

ABSTRACT: We calculate the generating functions of BPS indices using their modular properties in Type II and M-theory compactifications on compact genus one fibered CY 3 -folds with singular fibers and additional rational sections or just $N$-sections, in order to study string dualities in four and five dimensions as well as rigid limits in which gravity decouples. The generating functions are Jacobi-forms of $\Gamma_{1}(N)$ with the complexified fiber volume as modular parameter. The string coupling $\lambda$, or the $\epsilon_{ \pm}$parameters in the rigid limit, as well as the masses of charged hypermultiplets and non-Abelian gauge bosons are elliptic parameters. To understand this structure, we show that specific auto-equivalences act on the category of topological B-branes on these geometries and generate an action of $\Gamma_{1}(N)$ on the stringy Kähler moduli space. We argue that these actions can always be expressed in terms of the generic Seidel-Thomas twist with respect to the 6-brane together with shifts of the B-field and are thus monodromies. This implies the elliptic transformation law that is satisfied by the generating functions. We use Higgs transitions in F-theory to extend the ansatz for the modular bootstrap to genus one fibrations with $N$-sections and boundary conditions fix the all genus generating functions for small base degrees completely. This allows us to study in depth a wide range of new, non-perturbative theories, which are Type II theory duals to the CHL $\mathbb{Z}_{N}$ orbifolds of the heterotic string on $K 3 \times T_{2}$. In particular, we compare the BPS degeneracies in the large base limit to the perturbative heterotic one-loop amplitude with $R_{+}^{2} F_{+}^{2 g-2}$ insertions for many new Type II geometries. In the rigid limit we can refine the ansatz and obtain the elliptic genus of superconformal theories in $5 \mathrm{~d}$.

Keywords: D-branes, F-Theory, String Duality, Topological Strings

ARXIV EPRINT: 1910.01988 


\section{Contents}

1 Introduction 1

$\begin{array}{lll}2 & \text { The geometry of elliptic and genus one fibrations } & 7\end{array}$

3 Branes, derived equivalences and monodromies $\quad 9$

3.1 A brief introduction to topological B-branes 9

3.2 Fourier-Mukai transformations and monodromies 11

3.3 Relative conifold transformations and $\Gamma_{1}(N) \quad 13$

3.4 EZ-transformations and wall monodromies 16

3.5 When divisors collapse to curves 21

3.6 Monodromies and automorphic properties of $Z_{\text {top }} \quad 22$

4 The modular bootstrap for elliptic and genus one fibrations 25

4.1 Rings of modular forms and Jacobi forms for $\Gamma_{1}(N) \quad 26$

$\begin{array}{lll}4.2 & \text { The modular bootstrap for elliptic fibrations } & 29\end{array}$

4.3 Closed expressions for $Z_{\beta=0} \quad 31$

$\begin{array}{lll}4.4 & \text { Modular anomaly equations } & 35\end{array}$

4.5 The modular ansatz for genus one fibrations with $N$-sections 38

$\begin{array}{lll}\text { 4.6 Summary of the modular bootstrap ansatz } & 41\end{array}$

5 Duality with heterotic strings on CHL orbifolds 42

5.1 Heterotic/Type IIA duality and topological strings 42

5.2 Genus one fibrations and heterotic strings on $\left(K 3 \times T^{2}\right) / \mathbb{Z}_{N} \quad 43$

5.3 Constructing Calabi-Yau duals of CHL orbifolds 45

5.4 Comparison with the heterotic one-loop computation 48

6 Decoupling gravity on genus one fibrations over (-1)-curves 50

6.1 The refined topological string partition function 51

6.2 Genus one fibrations and E-strings with Wilson lines 52

7 Examples $\quad \mathbf{5 5}$

7.1 Eliptically fibered Calabi-Yau as toric hypersurfaces $\quad 55$

7.2 The Higgs chain $\mathrm{SU}(2) \rightarrow \mathrm{U}(1) \rightarrow \mathbb{Z}_{2}$

7.2.1 $M_{1}^{h}=\left(F_{4} \rightarrow \mathbb{P}^{2}\right)\left[\mathbb{Z}_{2}\right]_{2}^{-252} \quad 61$

7.2.2 $M_{2}^{h}=\left(F_{6} \rightarrow \mathbb{P}^{2}\right)[\mathrm{U}(1)]_{3}^{-216} \quad 65$

7.2.3 $M_{3}^{h}=\left(F_{10} \rightarrow \mathbb{P}^{2}\right)[\mathrm{SU}(2)]_{3}^{-216} \quad 67$

7.3 A genus-one fibrations over $\mathbb{F}_{1}:\left(F_{4} \rightarrow \mathbb{F}_{1}\right)\left[\mathrm{SU}(2) \times \mathbb{Z}_{2}\right]_{4}^{-144}$

8 Conclusion $\quad 71$

$\begin{array}{ll}\text { A A brief review of the F-theory dictionary } & 74\end{array}$

A.1 Gauge symmetries and matter in F-theory 74

A.2 The geometric origin of the anomaly polynomial 76

$\begin{array}{lll}\text { B An identity of characteristic classes } & 77\end{array}$ 
$\begin{array}{ll}\text { C Discriminants and Picard-Fuchs systems } & \mathbf{7 9}\end{array}$

$\begin{array}{ll}\text { C.1 }\left(F_{6} \rightarrow \mathbb{P}_{2}\right)[\mathrm{U}(1)]_{3}^{-216} & 79\end{array}$

C.2 $\left(F_{10} \rightarrow \mathbb{P}_{2}\right)[\mathrm{SU}(2)]_{3}^{-216} \quad 80$

D Toric data for geometries with local limits $\quad 81$

$\begin{array}{ll}\text { E Modular expressions for refined partition functions } & 83\end{array}$

$\begin{array}{lll}\text { F Refined BPS invariants } & \mathbf{8 4}\end{array}$

\section{Introduction}

In this paper we solve the all-genus topological string partition function $Z_{\text {top. }}$ on compact genus one fibered Calabi-Yau 3 folds $M$ in a large base expansion, extending the approach of $[1,2]$ to elliptic fibrations with reducible fibers and in particular to geometries that do not exhibit a section but only $N$-sections.

An elliptic curve is a genus one curve with a marked point which is the zero $\mathcal{O}$ in the additive group law on the elliptic curve [3]. An elliptic fibration over a base $B$ is accordingly defined to be a genus one fibration that has a global section which can be taken to define the zero $\mathcal{O}$ on each fiber and is called the zero section. The more general term genus one fibration refers to a geometry in which each fiber is a genus one curve, but no assumptions about the presence of a section is made.

Elliptic fibrations can have additional rational sections and they generate the ModellWeil group $\mathrm{MW}_{M}$ which is an Abelian group of finite rank that can have torsion parts. If a rational section corresponds to a torsion point $\mathcal{P}$ with $N \mathcal{P}=\mathcal{O}$ on each fiber one refers to it as an $N$-torsional section and the corresponding geometric group law on the fiber requires a specialized Weierstrass form of the Jacobian fibration [4]. On the other hand, independent non-torsional sections correspond to independent elements in the cohomology $H^{2}(M, \mathbb{Z})$. In the F-theory context it is well known that independent rational sections lead to an Abelian gauge group $\mathrm{U}(1)^{r-1}$ with $r=\operatorname{rk}\left(\mathrm{MW}_{M}\right)$ [5]. Explicit geometries were first discussed in [6] to resolve, in compact Calabi-Yau 3-folds, the BPS degeneracies of the $E$-string with respect to the flavour fugacities by breaking the flavour group $E_{8}$ on the $\frac{1}{2} K 3$ with $N-1$ global rational sections into $G$, where $\mathrm{U}(1)^{N-1} \times G \subset E_{8}$ and $G=\left\{E_{7}, E_{6}, D_{5}, \ldots\right\}$ for $N=2,3,4, \ldots$

An important class of fibrations that is discussed in this paper has no section but just $N$-sections. An $N$-section is defined by $N$ points that can be identified on each fiber and which are transformed into each other by monodromies in the base. Accordingly, an $N$ section corresponds to one element in the cohomology $H^{2}(M, \mathbb{Z})$. Genus one-fibrations that have no section but only $N$-sections lead to discrete $\mathbb{Z}_{N}$ gauge symmetries in F-theory. The corresponding effective theories can be obtained via Higgsing from vacua with U(1) factors and matter multiplets of charge $N$, a mechanism first described geometrically in [7, 8]. Again, $N$-sections that are in an appropriate sense independent lead to Abelian gauge bosons. When we say that $M$ is a genus one fibration with $N$-sections we will imply that it does not have a section and also no $N^{\prime}$-sections with $N^{\prime}<N$. 
In addition to rational sections or $N$-sections, a genus one fibration can exhibit fibral divisors that resolve $A D E$-singularities that are fibered over curves in the base. There can be also global monodromies that identify nodes of the $A D E$-singularities and lead to the reduced number of fibral divisors of non-simply laced gauge groups [9]. The corresponding harmonic forms in $H^{2}(M, \mathbb{Z})$ lead to the Abelian gauge bosons in the Cartan of the corresponding gauge group while, from the M-theory perspective, wrapped 2-branes correspond to the $W$-bosons. After compactifying the F-theory vacuum on a circle, the volumes of components of reducible fibers can be identified with scalar fields in vector multiplets of the corresponding $\mathcal{N}=2$ theories. In the effective theory they parametrize the masses of charged hypermultiplets and of the non-Abelian $W$-bosons. We therefore denote these parameters generically by $\underline{m}$ and, for reasons that we outline below, we call them geometric elliptic parameters.

In $[1,2]$ it was assumed that $M$ is an elliptic fibration which has just the zero section and exhibits no fiber singularities more severe than $I_{1}$ in Kodaira's classifications. In particular, all fibers were irreducible and no fibral divisors have been present. It was then shown that the expansion coefficients ${ }^{1} Z_{\beta}(\tau, \lambda)$ of the topological string partition function $Z_{\text {top. }}$ on elliptic Calabi-Yau 3-folds for fixed classes $\beta \in H_{2}(B, \mathbb{Z})$ in the base are meromorphic Jacobi forms of weight zero, where the elliptic argument $\tau$ is related to the complexified volume of the elliptic fiber, while the string coupling constant $\lambda$ appears as elliptic argument. Moreover, $Z_{\beta}(\tau, \lambda)$ has an index that is given by intersections on the base as $r_{\lambda}^{\beta}=\frac{1}{2} \beta \cdot\left(\beta-c_{1}(B)\right)$. The former fact can be argued using invariance of $Z_{\text {top. }}$ under an $\mathrm{SL}(2, \mathbb{Z})$ action that is embedded into the symplectic monodromy group $\Gamma_{W} \subset S p\left(b_{3}(W), \mathbb{Z}\right)$ acting on the integral symplectic basis of periods of the mirror $W$ of the elliptic fibration $M$. The index follows by combining this action with the background independence equation of $Z_{\text {top. }}$ as a wave function [1, 10, 11], which is equivalent to the holomorphic anomaly equation. Using the pole structure of $Z_{\beta}$ imposed by the integral BPS expansion of $Z_{\text {top. }}$ one can argue that $Z_{\beta}$ has a unique denominator and the numerator is a weak Jacobi form. The ring of weak Jacobi forms is finitely generated, see [1] and in particular reference [12]. Therefore one can fix $Z_{\beta}$ for low base degree from the vanishing of BPS invariants for high genus that follow from Castelnuovo bounds. ${ }^{2}$

A crucial step in generalizing the analysis of $[1,2]$ is to modify and extend these monodromy arguments to genus one fibrations that do not have a section and also to include those parameters that correspond to the volumes of fibral curves and, after circle compactification, to the vaccum expectation values of scalars in vector multiplets. The action of the corresponding monodromies for elliptic fibrations with reducible fibers has been calculated in [13] and we extend the argument to fibrations without sections. Using this generalization, we establish that for genus one fibrations with $N$-sections and $N \leq$ 4 , the $\operatorname{SL}(2, \mathbb{Z})$ part is broken to the finite index subgroup $\Gamma_{1}(N)$ as well as the fact that the complexified Kähler parameters that correspond to rational fibral curves become

\footnotetext{
${ }^{1}$ See $(2.3)$ (without the mass parameters $\underline{m}$ ) for the precise definition of the expansion.

${ }^{2}$ If $\beta^{2}<0$ one can argue that one can always fix $Z_{\beta}$ [1] and the method is quite effective. For example the E-string could be solved to seven base windings in [2].
} 
elliptic parameters of the coefficients $Z_{\beta}(\tau, \lambda, \underline{m}){ }^{3}$ This analysis enables us to construct the $Z_{\beta}(\tau, \lambda, \underline{m})$ in a far more general setting as meromorphic higher degree Jacobi form of $\Gamma_{1}(N)$ with further elliptic parameters, where the numerators are now generated by the larger rings of weak Jacobi-forms under $\Gamma_{1}(N)$, which exhibit Weyl-invariance in the $\underline{m}$ parameters similar to the cases discussed [19]. The monodromies allow us to determine the precise index matrix with respect to the geometric elliptic parameters $\underline{m}$ and the elliptic transformation law then follows for these parameters already from invariance of the topological string partition function under the corresponding monodromies. Although the rings of weak higher degree Jacobi forms for $\Gamma_{1}(N)$ are larger, we show in examples that one can fix $Z_{\beta}(\tau, \lambda, \underline{m})$ for small $\beta$ and expect that the Castelnuovo bounds are again sufficient to solve completely for classes with $\beta^{2}<0$.

Calculating the monodromy group $\Gamma_{W} \in \operatorname{Sp}\left(b_{3}(W), \mathbb{Z}\right)$ in the B-model on $W$ using the complete Picard Fuchs differential ideal and a global integral symplectic basis on the resolved complex structure moduli space $\mathcal{M}_{c s}(W)$ is technically feasible only for models with few complex moduli $h_{2,1}(W)$. Instead we work on the stringy Kähler moduli space $\mathcal{M}_{k s}(M)$ of $M$ directly. Extending the method of [2, 13, 23], our strategy is to identify the Kähler moduli as central charges of 2-branes and to study auto-equivalences of the category of branes that generate an action of $\Gamma_{1}(N)$. We will then relate those auto-equivalences to the generic monodromies that correspond to the boundary of the geometric cone and to the large volume limiting points and thus show that the $\Gamma_{1}(N)$ action corresponds indeed to monodromies in the stringy Kähler moduli space.This allows us to use the known automorphic properties of $Z_{\text {top. }}$ under monodromies $[24,25]$ and to derive the elliptic transformation law with respect to the geometric elliptic parameters and thus also to identify their index matrix.

For elliptic fibrations, the most characteristic auto-equivalence is induced by the Fourier-Mukai kernel which is the ideal sheaf $\mathcal{I}_{\Delta_{B}}$ of the relative diagonal $\Delta_{B}$ in $M \times_{B}$ $M[26-30]$ and it acts as $\tau \rightarrow \frac{\tau}{N \tau+1}$ on the $\tau$ parameter [13]. We will call this the $U$ transformation or the relative Conifold transformation. Furthermore, tensoring objects in the derived category with the line bundle $\mathcal{O}(D)$, where $D$ is an effective Cartier divisor, also induces an auto-equivalence and corresponds to a shift of the $B$ field by $D$. In partiular, when $D$ is choosen to be the "zero" $N$-section, it leads to the $T$ transformation $\tau \rightarrow \tau+1$. The Weyl symmetries in the geometric elliptic parameters $\underline{m}$ arise from monodromies around points where the Calabi-Yau develops a singularity from divisors collapsing to curves [31, 32]. The corresponding Fourier-Mukai kernels have been indentified in [33-35], see also [36].

After general explanations of the geometric structure of the fibration at the beginning of section 3, the central charges and the symplectic pairing of the topological $B$-branes are explained in subsection 3.1. The Fourier-Mukai kernels and the corresponding actions on the brane charges are described and their action on the brane charges is calculated in the subsections $3.2,3.3$. The calculation of the $U$-transformation generalizes the re-

\footnotetext{
${ }^{3}$ In the rigid limits the masses appear as fugacities in the elliptic genus of the $2 \mathrm{~d}$ gauged linear quiver $\sigma$ model and are naturally identified as elliptic parameters [14-20]. In global threefold cases with multiple sections, a similar behaviour has been observed in [21, 22].
} 
sults from [13] to genus one fibrations with $N$-sections and relies on a realization of the Calabi-Yau as a complete intersection inside a compatibly fibered toric ambient space. In subsection 3.4 we argue that the $U$-transformation can always be expressed in terms of the generic Seidel-Thomas twist with respect to the 6-brane together with shifts of the B-field and is thus a monodromy. For fibrations with bases $B=\mathbb{P}^{2}$ and $\mathbb{F}_{n}, n \in \mathbb{N}$ we provide the relations and check them via an explicit calculation of the corresponding products of monodromies. This calculation does not rely on toric geometry and applies to all genus one fibered Calabi-Yau threefolds over the corresponding bases. At the end of subsection 3.4 we also discuss a beautiful connection of this structure to the swampland distance and the emergence conjectures. The Weyl monodromies that emerge when divisors collapse to curves are discussed in subsection 3.5. In subsection 3.6 we then combine the monodromies with the automorphic properties of the topological string partition function $Z_{\mathrm{top}}$. and derive the elliptic transformation law. A derivation of the full modular transformation law is given for genus one fibrations without additional $N$-sections or fibral divisors, and where geometric elliptic parameters are thus absent.

We explain in section 4 how to use the modular and elliptic transformations of weight $k=0$ and index $r_{\lambda}^{\beta}$ under $\Gamma_{1}(N)$ as well as the pole behaviour to reconstruct $Z_{\beta}$. For convenience of the reader we include in subsection 4.1 the definitions of the rings of holomorphic modular forms of $\Gamma_{1}(N)$ and discuss the special class of higher degree Jacobi forms that will appear in the topological string partition function. Subsection 4.2 contains a review of the main results about the modular bootstrap on elliptic fibrations. In subsection 4.3 we then discuss the base degree zero part $Z_{0}$ of the partition function, both on elliptic fibrations with reducible fibers and on genus one fibrations with $N$-sections. We find that the corresponding free energies $F_{g \geq 0, \beta=0}$ are Jacobi forms of weight $2 g-2$ and index 0 . For particular examples we derive the closed expression at all genera and the derivation can easily be adapted to other genus one fibrations. In particular, the matter content of the corresponding F-theory vaccum including multiplicities is entirely encoded in $Z_{0} \cdot{ }^{4}$ Subsection 4.4 contains the derivation of the index with respect to the topological string coupling constant $\lambda$, using Witten's form of the wave function equation. We then obtain the correct Ansatz for general $Z_{\beta}$ on general genus one fibered Calabi-Yau threefolds with $N$-section from the corresponding Ansatz for elliptic fibrations by considering Higgs transitions in F-theory, see subsection 4.5. We summarize the equations relevant for all modular Ansätze in subsection 4.6.

Of course, the full topological partition function contains much richer BPS information than just the massless spectrum of the corresponding F-theory vacuum. A beautiful application to Type II/heterotic duality for Calabi-Yau 3-folds with $N$-section geometries in four dimensional $\mathcal{N}=2$ theories is described in section 5 . On the heterotic side one expects $[39,40]$ the dual to be a CHL $[41,42]$ compactifictation on $\left(K 3 \times T_{2}\right) / \mathbb{Z}_{N}$. This follows from the observation that self-dualities on the heterotic side are identified with monodromies in the moduli space of the Calabi-Yau target space on the Type II side [43, 44],

\footnotetext{
${ }^{4}$ Such observations have been independently made in the elliptic case by Amir Kashani-Poor [37] and they will also expanded in [38].
} 
together with our general discussion of the monodromies of genus one fibrations. The $\mathbb{Z}_{2}$ CHL string exists already for $S^{1}$ compactifications of the $E_{8} \times E_{8}$ heterotic string and the corresponding $\mathbb{Z}_{2}$ exchanges the two $E_{8}$ factors while acting as a half shift $x_{k} \rightarrow x_{k}+\pi R$ on the circle. Using a maximal supersymmetric dual pair in $6 \mathrm{~d}$ between a Type II $\mathbb{Z}_{2}$ orbifold on $K 3$ and a heterotic CHL $\mathbb{Z}_{2}$ orbifold on $T^{4}$ [45], as well as an adiabatic argument on the Type II side, the $\mathbb{Z}_{2}$ action on a dual elliptically fibered Calabi-Yau 3-fold was identified in [39]. Indeed, this leads to a 2 -section geometry with a compatible $K 3$ fibration.

The automorphisms of $K 3$ surfaces have been classified [46] and correspond to conjugacy classes of the Mathieu group $M_{23}$. More generally, one can classify the discrete symmetries of non-linear sigma models on $K 3$, a task completed in [47, 48]. Using this information in further $T^{2}$ compactifictions one can fully classify orbifolds of Type II compactifications on $K 3 \times T^{2}$ which yield $\mathcal{N}=4$ supergravities in four dimensions ${ }^{5}$ [49]. One can also classify CHL orbifolds of heterotic strings on $K 3 \times T^{2}$ which lead to $\mathcal{N}=2$ effective supergravity actions $[50,51]$. For the corresponding Type II theories on Calabi-Yau 3-folds with $N$-sections and compatible $K 3$ fibration one should be able to identify the all-genus BPS amplitudes with a heterotic one loop integral that contains insertions of the self-dual parts of curvature and graviphoton field strength of the form $R_{+}^{2} F_{+}^{2 g-2}$ [52-54]. The corresponding perturbative heterotic amplitudes can be calculated using the Borcherds lift as in $[54,55]$. Similar calculations have been performed for some CHL models in [50, 51]. In subsection 5.4 we identify novel Type II compactifications which are dual to heterotic compactifications on CHL orbifolds that correspond to the conjugacy class $2 A$ in $M_{23}$ with non-standard embedding of the gauge connection. We show that the corresponding oneloop amplitudes that were obtained in [51] match our results from the modular bootstrap on genus one fibrations in the large base limit of the compatible $K 3$ fibration and thereby provide a strong all genus tests of Type II/CHL duality. Since the heterotic dilaton $\Phi_{\text {het }}$ is identified with the Kähler parameter of the base of the $K 3$ fibration, instanton corrections with non-vanishing base degree contribute like $q_{b}=e^{-8 \pi^{2} \Phi_{h e t}}$ and predict non-perturbative corrections to the CHL string. Type II theory on $N$-section Calabi-Yau 3-folds should give therefore the full non-perturbative description of the corresponding CHL string.

An analysis of superconcormal representation theory reveals, that six is the maximal dimension in which superconformal field theories can exist [56]. F-theory on fibrations with a contractable configuration of curves in a non-compact base have been used to study $[5,6]$ and classify $N=(2,0)$ [57] and $N=(1,0)$ [58] superconformal field theories. Building blocks in this classifications are minimal SCFTs studied in [15] and among the latter is a particularly interesting one, the so called $E$-string. This is geometrically engineered as the rigid F-theory limit on an elliptic Calabi-Yau 3-fold with a rational curve of self-intersection -1 in the base. The limit decouples gravity and emerges geometrically when the normal direction of the rational curve is decompactified, so that the compact part of the geometry is an $\frac{1}{2} K 3$, i.e. an elliptic surface with $12 I_{1}$ fibers. The latter can also be obtained as a nine-fold blowup of $\mathbb{P}^{2}[5,6]$. The topological string partition function encodes the elliptic genera of tensionless strings that arise in the superconformal limit [15].

\footnotetext{
${ }^{5}$ This setting is very well studied in order to understand the microscopic entropy of $\mathcal{N}=4$ black holes and because the twining elliptic genera are given by Jacobi forms, whose coefficients decompose in a simple way into the dimensions of the largest exceptional discrete groups, a phenomenon known as moonshine.
} 
In section 6 we study corresponding gravity decoupling limits now in the context of genus one fibrations with $N$-sections. To this end we use toric geometry to construct genus one fibrations over the Hirzebruch surface $\mathbb{F}_{1}$. The latter is a rational fibration with fiber $F \sim \mathbb{P}^{1}$ with $F^{2}=0$ and base $S \sim \mathbb{P}^{1}$, a section with $S^{2}=-1$. The decoupling limit corresponds hence to the limit of large fiber $F$, or equivalently to contracting the base $S$. Taking this limit on genus one fibrations with $N$-sections ${ }^{6}$ over $\mathbb{F}_{1}$, we find for $N=2,3,4$ that, due to the additional $\mathrm{U}(1)_{R}$ symmetry in the local limit, the $Z_{k \cdot S}(\tau, \lambda)$ can be refined for $k$-th multi wrapping $\beta=k \cdot S$ of the base class $S$ to $Z_{k \cdot S}\left(\tau, \epsilon_{ \pm}\right)$. Adapting the refined modular bootstrap approach with $\operatorname{SL}(2, \mathbb{Z})$ Jacobi forms with two elliptic parameters $\epsilon_{ \pm}$ described in [18] to similar Jacobi forms of $\Gamma_{1}(N)$ we can extract the refined BPS invariants $N_{j_{L}, J_{R}}^{\beta}$ in the full $5 \mathrm{~d}$ spin representations of the little group $\mathrm{SU}(2)_{L} \times \mathrm{SU}(2)_{R}$ as decribed in subsection 6.2. In particular, to determine the elliptic index with respect to the refined parameters $\epsilon_{ \pm}$, we use the relation of the latter to the anomaly polynomials for the chiral $6 d$ space time - or quiver worldsheet theory $[17,19]$. The BPS spectrum can be explained by discrete Wilson lines on the $S^{1}$ that compactifies from six to five dimension. In particular for $N=2$ we find the refined BPS invariants of the $5 \mathrm{~d}$ theory with an $\mathbb{Z}_{2}$ discrete Wilson line that was used in [59]. The latter was used to obtain the $E$-string spectrum, up to the effect of the Wilson line, from the elliptic genus of a $2 \mathrm{~d}$ quiver gauge theory with $\mathrm{SO}(16)$ gauge theory, which has a brane description as $k D 2$ branes stretched between a stack of one 08 - and $8 D 8$ branes and one NS5-brane.

Our toric construction of genus one fibered Calabi-Yau 3-folds over various bases with $N$-sections as well as with Abelian and non-Abelian gauge symmetry enhancements is illustrated with typical examples in section 7 . There we apply the modular bootstrap in detail and also discuss the manifestation of field theoretic Higgs transitions at the level of the geometry and the partition function. We provide the toric data related to the ambient space, study the Mori cone in the relevant phases and obtain the Picard-Fuchs differential ideals as well as the discriminants. Longer expressions are relegated to appendix D and C. From the toric data mirror symmetry is manifest [60,61] and the periods at large radius as well as the genus zero BPS invariants can be calculated from the GKZ solutions as in $[62,63]$. If all discriminant components are known, the genus one BPS invariants follow as in [64].

We provide auxiliary data for our discussion in the appendices. Moreover, appendix A contains a very brief review of the F-theory dictionary that we frequently employ to describe geometric structures in terms of the physics in the corresponding F-theory vacua. In appendix $\mathrm{B}$ we prove an identity between charateristic classes that is crucial to obtain the auto-equivalences and monodoromies in subsection 3.3 as well as in the derivation of the modular anomaly equation in subsection 4.4.

\footnotetext{
${ }^{6}$ We also consider geometries with pseudo $N$-sections that nevertheless exhibit $\Gamma_{1}(N)$ modularity. Those occur when the Kähler moduli of the one or two rational sections considered for the $E_{7} \times \mathrm{U}(1)$ and $E_{6} \times \mathrm{U}(1)^{2}$ splitting of the $E$-string [6] are frozen to zero value by the toric embedding.
} 


\section{Acknowledgments}

We would like to thank Min-xin Huang and Sheldon Katz for discussions and the collaboration on compact elliptic fibrations using the modular bootstrap and Michelle del Zotto, Jie $\mathrm{Gu}$, Amir Kashani-Poor, Gugielmo Lockhart and Cumrun Vafa for sharing insights into the non-compact cases. Special thanks goes to Xin Wang for checking the compatibility of the refined local limits of the $N$-section cases with the blow up equations. We also thank Paul Oehlmann for insightful discussions regarding the physics of compactifications on genus one fibrations. We thank Johanna Knapp and David Erkinger for valuable discussions about D-brane monodromies and their realizations in the gauged linear sigma model, as well as the swampland distance program in the context of fibrations. The work of Thorsten Schimannek is supported by the Austrian Science Fund (FWF):P30904-N27. Cesar Fierro Cota would like to thank the financial support from the fellowship "Regierungsstipendiaten CONACYT-DAAD mit Mexiko" under the grant number 2014 (50015952) and the Bonn-Cologne Graduate School of Physics and Astronomy for their generous support.

\section{The geometry of elliptic and genus one fibrations}

Before we begin, let us briefly review the geometry of an elliptic or genus one fibered Calabi-Yau threefold. Note that we follow the convention from the F-theory literature that elliptic fibrations have at least one section and use the word genus one fibration for torus fibrations that do not necessarily have a section. We will always assume that the fibration is flat (i.e. the dimension of the fiber does not jump) and that there are no multiple fibers.

The Shioda-Tate-Wazir theorem [65] states that the homology group $H_{4}(M)$ of an elliptically fibered threefold is generated by three types of divisors. These are vertical divisors $D_{i}=\pi^{-1} \tilde{D}_{i}$ where $\tilde{D}_{i} \in H_{2}(B)$ is a divisor in the base $B$ of the fibration, fibral divisors that consist of rational curves fibered over a divisor in $B$ and sections. It is useful to distinguish between holomorphic sections, that intersect every fiber in a point, and rational sections, that intersect every smooth irreducible fiber in a point. By convention an elliptic fibration has at least one section, holomorphic or rational, and we can choose any section to be the zero section. Irreducible fibers can then be canonically identified with $\mathbb{C} /(\mathbb{Z}+\tau \mathbb{Z})$ where the origin is the point that corresponds to the intersection with the zero section. Addition of points defines a group law on the fiber that can be extended to the set of rational sections. This leads to the Mordell-Weil group $M W(M)$. If we only consider the zero section and sections that are linearly independent in the Mordell-Weil group $M W(M)$ together with a basis of vertical divisors and fibral divisors we obtain a basis of $H_{4}(M)$.

In our convention a genus one fibration might not have a section but only $k$-sections that intersect the generic fiber $k$ times. The points experience monodromy along loops in the base which distinguishes a $k$-section from a union of $k$ sections. However, a genus one fibration has an associated Jacobian fibration where every fiber is replaced by its moduli space of degree 0 line bundles. Note that an elliptic fibration is birationally equivalent to it's associated Jacobian fibration and on smooth fibers the zero section can be identified 
with the trivial line bundle. The Mordell-Weil group law then locally amounts to taking tensor products. In the case of genus one fibrations one can identify the $k$-sections with degree $k$ line bundles on the (smooth) fibers. It is then clear that the Mordell-Weil group of the Jacobian fibration acts on the set of $k$-sections.

The Shioda-Tate-Wazir theorem has subsequently been generalized to genus one fibered threefolds, i.e. fibrations that do not admit a section but only multi-sections [66]. The only difference is that one considers $k$-sections (for minimal $k$ ) instead of sections. To obtain a basis one picks a "zero $k$-section" and acts on it with the free generators of the Mordell-Weil group of the Jacobian fibration.

The homology of an elliptic or genus-one fibered Calabi-Yau manifold, including the intersection structure, is encoded in the effective theory that is associated to the Calabi-Yau via F-theory. This provides a concise way to describe e.g. the group of divisors in terms of the corresponding physical gauge group. We will frequently make use of this dictionary and a brief review of the relevant entries can be found in appendix A.

For elliptically fibered Calabi-Yau manifolds there is a homomorphism from the Mordell-Weil group to $H_{4}(M, \mathbb{Z})$ called the Shioda map

$$
\sigma: M W(M) \rightarrow H_{4}(M, \mathbb{Z}) .
$$

The explicit form of this map is reviewed in appendix A but one can uniquely define it in terms of it's intersection properties [67]. This definition generalizes to genus one fibrations with $N$-sections where the domain is of course just a set. Let us define the inner product

$$
\langle,\rangle: H_{4}(M) \times H_{4}(M) \rightarrow H_{2}(B), \quad\left(S, S^{\prime}\right) \mapsto-\pi\left(S \cdot S^{\prime}\right)
$$

For an $N$-section $E$ of a Calabi-Yau threefold we then define $\sigma(E)=E+D$ where $D$ is the unique linear combination of the zero- $N$-section, vertical divisors and fibral divisors such that $\sigma(E)$ is orthogonal with respect to $\langle\cdot, \cdot\rangle$ to the subspace spanned by those divisors in $H_{4}(M)$.

Irreducible curves in $M$ can either arise from curves in the base, from rational curves that are fibers of fibral divisors or from isolated rational curves over points of the base. We will denote the latter two collectively as fibral curves. It is easy to see that the image of an $N$-section under the Shioda map $\sigma$ will have non-zero intersection only with isolated fibral curves. The class of the generic fiber is irreducible if there are no reducible fibers. We can therefore expand the topological string partition function $Z=\exp \left(\sum_{g=0}^{\infty} \lambda^{2 g-2} F_{g}\right)$ as

$$
Z(\tau, \underline{m}, \underline{t}, \lambda)=Z_{0}(\tau, \lambda)\left(1+\sum_{\beta \in H^{1,1}(B, \mathbb{Z})} Z_{\beta}(\tau, \underline{m}, \lambda) Q^{\beta}\right),
$$

where we find that for an elliptic or genus one fibration with $N$-sections for $N \in\{1,2,3,4\}$, the Kähler modulus $\tau$ should be choosen such that $N \tau$ is the complexified volume of the generic fiber (this is discussed in section 4.5). Moreover, $\underline{m}$ are complexified volumes of fibral curves and $Q^{\beta}=\exp \left(2 \pi i \sum_{i} \beta^{i} t_{i}\right)$ where $t_{i}, i=1, \ldots, h^{1,1}(B)$ are shifted volumes of curves in the base. The shift is linear in $\tau$ and its necessity for elliptic fibrations has first 
been observed by $[11,68]$. It can also be derived from the action of the relative conifold transformation [13]. We will extend this derivation to genus one fibrations in section 3.3. The topological string coupling constant is denoted by $\lambda$.

\section{Branes, derived equivalences and monodromies}

In this paper we adopt the philosophy that modular properties of the topological string partition function are consequences of the general transformation behaviour under monodromies in the stringy Kähler moduli space. Here the attribute "stringy" indicates two important differences to the classical Kähler moduli space of a Calabi-Yau. First, the Kähler form is combined with the B-fields into the complexified Kähler form. Second, the complexified Kähler cone is extended with the Kähler cones of other geometries and cones that do not admit a geometric interpretation [69, 70]. A canonical example for such a non-geometric "phase" is the moduli cone of a Landau-Ginzburg model. Together these cones form the so-called fully enlarged Kähler moduli space. The third difference is, that the duality group of the string compactification is quotiented out.

At least for hypersurfaces in toric ambient spaces it is relatively easy to construct the fully enlarged Kähler moduli space. It is much harder to decide whether two points correspond to dual compactifications. However, according to the homological mirror symmetry conjecture the action of monodromies in the stringy Kähler moduli space, where we choose the large volume limit of a Calabi-Yau $M$ as the base point, can be lifted to autoequivalences of the category of topological B-branes on $M$ [71]. This allows us to use the machinery of Fourier-Mukai transformations and thus to calculate the action of certain generic autoequivalences on the complexified Kähler moduli.

Our main interest will be in what we call the relative conifold transformation on elliptic and genus-one fibered Calabi-Yau threefolds. For fibrations over $\mathbb{P}^{2}$ we show that the action on the brane charges can be expressed in terms of transformations that are related to monodromies in the complex structure moduli space of the mirror. This implies that the corresponding points in the Kähler moduli space are identified by a duality. In the case of elliptic fibration this duality is closely related to T-dualizing along both cycles of the elliptic fiber. When the base is a Hirzebruch surface $\mathbb{F}_{i}, i=0,1$ we observe analogous relations for all geometries that we study in this paper. Having thus established that relative Conifold transformations correspond to monodromies in the stringy Kähler moduli space, we relate it to the modular properties of the topological string partition function.

We start this section with a cursory review of topological B-branes and Fourier-Mukai transformations where we will also introduce our conventions. A pedagogical review can be found in [72].

\subsection{A brief introduction to topological B-branes}

Topological B-branes on a Calabi-Yau manifold $n$-fold $M$ are objects in the bounded derived category of quasicoherent sheaves $D^{b}(M)$ on $M$ [73]. An object $\mathcal{F}^{\bullet}$ in this category is a bounded complex of quasicoherent sheaves and quasi-isomorphic complexes are identified. 
This implies (non-trivially) that every object can be represented by a bounded complex of locally free sheaves

$$
\mathcal{F}^{\bullet}=0 \rightarrow \mathcal{E}^{m} \rightarrow \mathcal{E}^{m+1} \rightarrow \cdots \rightarrow 0
$$

where the superscript indicates the position in the complex.

Locally free sheaves are equivalent to vector bundles and the complex (3.1) can be thought of as a stack of $n$-branes (at even positions) and anti- $n$-branes (at odd positions in the complex). The identification of objects in the derived category implements the fact that via brane/anti-brane annihilation many stacks are identified in the infrared limit. In particular, branes that do not wrap the Calabi-Yau correspond to quasicoherent sheaves that are not locally free and can be realized by annihilating brane/anti-brane pairs with lower-dimensional branes dissolved on their world volumes.

The RR charges of B-branes are classified by $K$-theory or, since we are only interested in geometries without torsion, by the vertical cohomology on $M$. For a B-brane $\mathcal{F}^{\bullet}$ the asymptotic central charge can be calculated using the Gamma class formula [74]

$$
\Pi_{\text {asy }}\left(\mathcal{F}^{\bullet}\right)=\int_{M} e^{\omega} \Gamma_{\mathbb{C}}(M)\left(\operatorname{ch} \mathcal{F}^{\bullet}\right)^{\vee},
$$

where in terms of the Chern classes $c_{2}, c_{3}$ of $M$

$$
\Gamma_{\mathbb{C}}(M)=1+\frac{1}{24} c_{2}+\frac{\zeta(3)}{(2 \pi i)^{3}} c_{3} .
$$

The action of the operator $\vee: \oplus_{k} H^{k, k}(M) \rightarrow \oplus_{k} H^{k, k}(M)$ is linear and determined by $\delta^{\vee} \mapsto(-1)^{i} \delta$ for $\delta \in H^{i, i}(M)$.

It has been conjectured by Kontsevich that mirror symmetry relates B-branes on $M$ to A-branes on the mirror $W$ [71]. The latter are calibrated Lagrangian $k$-cycles $L$ and the central charge is determined by the holomorphic $(3,0)$-form $\Omega$ as

$$
\Pi(L)=\int_{L} \Omega .
$$

The mirror map then identifies the asymptotic central charge of $\mathcal{F}^{\bullet}(3.2)$ with the logarithmic terms in the central charge (3.4) of the mirror brane $L$. Furthermore, the intersection between two A-branes $L_{1} \cap L_{2}$ is equal to the open string index of the mirror branes

$$
\chi\left(\mathcal{E}^{\bullet}, \mathcal{F}^{\bullet}\right)=\int_{M} \operatorname{Td}(M) \operatorname{ch}\left(\mathcal{E}^{\bullet}\right)^{\vee} \operatorname{ch}\left(\mathcal{F}^{\bullet}\right),
$$

where the Todd class $\operatorname{Td}(M)$ can be expressed in terms of the Chern classes via

$$
\operatorname{Td}(M)=1+\frac{1}{2} c_{1}(M)+\frac{1}{12}\left(c_{1}(M)^{2}+c_{2}(M)\right)+\frac{1}{24} c_{1}(M) c_{2}(M)+\ldots
$$

Of course, for a Calabi-Yau manifold $c_{1}(M)=0$ and most of the terms vanish. 
We will now introduce a set of branes that generate the charge lattice. The structure sheaf $\mathcal{O}_{M}$ corresponds to a 6-brane, while the skyscraper sheaf $\mathcal{O}_{p}$ with support on some point $p \in M$ corresponds to a 0 -brane. The short exact sequence

$$
0 \rightarrow \mathcal{O}_{M}(-D) \rightarrow \mathcal{O}_{M} \rightarrow \mathcal{O}_{D} \rightarrow 0
$$

implies a quasi-isomorphism between a 4 -brane $\mathcal{O}_{D}$ with support on the effective Cartier divisor $D$ and the complex of vector bundles

$$
\mathcal{F}_{D}^{\bullet} \equiv 0 \rightarrow \mathcal{O}_{M}(-D) \rightarrow \mathcal{O}_{M} \rightarrow 0
$$

The position of the brane is encoded in the maps of the complex but will not be important to us. Two-branes with support on a curve $C$ can be obtained as K-theoretic push-forwards

$$
\mathcal{C}^{\bullet}=\iota_{!} \mathcal{O}_{C}\left(K_{C}^{1 / 2}\right) \text {. }
$$

Let us assume a basis of the Kähler cone is given by $J_{i}, i=1, \ldots, h^{1,1}(M)$ and denote the dual curves by $C_{i}=1, \ldots, h^{1,1}(M)$. We expand the Kähler form $\omega$ as

$$
\omega=J_{i} t^{i}
$$

and, using the Gamma class formula (3.3), calculate the asymptotic central charges (see e.g. [75] for details of the calculation)

$$
\begin{aligned}
\Pi_{\text {asy }}\left(\mathcal{O}_{M}\right) & =\frac{1}{6} c_{i j k} t^{i} t^{j} t^{k}+b_{i} t^{i}+\frac{\zeta(3)}{(2 \pi i)^{3}} \chi, \\
\Pi_{\text {asy }}\left(\mathcal{F}_{J_{i}}^{\bullet}\right) & =-\frac{1}{2} c_{i j k} t^{j} t^{k}-\frac{1}{2} c_{i i j} t^{j}-\frac{1}{6} c_{i i i}-b_{i}, \\
\Pi_{\text {asy }}\left(\mathcal{C}_{i}^{\bullet}\right) & =t^{i}, \quad \Pi_{\text {asy }}\left(\mathcal{O}_{p}\right)=-1,
\end{aligned}
$$

where $\chi$ is the Euler characteristic of $M$ and we introduced

$$
c_{i j k}=\int_{M} J_{i} J_{j} J_{k} \quad \text { and } \quad b_{i}=\frac{1}{24} \int_{M} c_{2} J_{i} .
$$

In the remainder of this paper we will refer to the central charges of this basis as

$$
\vec{\Pi}=\left(\Pi^{(6)}, \Pi_{i}^{(4)}, \Pi_{i}^{(2)}, \Pi^{(0)}\right)^{t},
$$

and the Calabi-Yau $M$ as well as the choice of basis for the Kähler cone will be clear from the context.

\subsection{Fourier-Mukai transformations and monodromies}

The periods of the holomorphic $(k, 0)$-form can be calculated to arbitrary order in the complex structure parameters of $W$ and it is well known that they experience monodromy when transported along non-contractible loops in the complex structure moduli space. The homological mirror symmetry conjecture implies that the B-model monodromies lift, in the A-model, to auto-equivalences of the category of B-branes. On the other hand, 
a theorem by Orlov [76] states that equivalences of derived categories $D^{b}(X), D^{b}(Y)$ are always expressible as Fourier-Mukai transformations

$$
\Phi_{\mathcal{E}}: \mathcal{F}^{\bullet} \mapsto R \pi_{1 *}\left(\mathcal{E} \otimes_{L} L \pi_{2}^{*} \mathcal{F}^{\bullet}\right)
$$

where $\pi_{i}, i=1,2$ are the projections from $Y \times X$ to the $i$-th factor and the so-called Fourier-Mukai kernel $\mathcal{E}$ is a quasicoherent sheaf on $Y \times X$. The letters $L$ and $R$ indicate that the corresponding derived version of a functor is to be taken.

It is, in general, difficult to evaluate this expression and it is often easier to obtain the corresponding action on the brane charges. This can be done with the GrothendieckRiemann-Roch formula which for $f: X \times Y \rightarrow Y$ states that

$$
\operatorname{ch}\left(f_{*} \mathcal{F}^{\bullet}\right)=f_{*}\left[\operatorname{ch}\left(\mathcal{F}^{\bullet}\right) \cdot f^{*} \operatorname{Td}(X)\right] .
$$

Here we already assume that $X \times Y$ and $Y$ are smooth and neither of the associated K-groups contains torsion such that we can work directly in cohomology.

For several important B-model monodromies the corresponding Fourier-Mukai kernel is known to be of a generic form which allows us to calculate the corresponding action on the brane charges. We will now discuss the transformations that are most relevant for our discussion.

Large volume monodromies. Let us denote the embedding of the diagonal $\Delta=M$ into $M \times M$ by $j: M \rightarrow M \times M$. The Fourier-Mukai kernel that corresponds to the large volume monodromy around the divisor in Kähler moduli space where we move to infinity in the direction of a divisor $D$ inside the Kähler cone is given by $j_{*} \mathcal{O}(D)$ [77]. The general formula (3.14) can be evaluated (see e.g. [36, 78]) and one finds that the transformation maps

$$
\mathcal{F}^{\bullet} \mapsto \mathcal{F}^{\bullet} \otimes \mathcal{O}(D)
$$

At the level of the central charges this transformation just leads to a shift in the classical/logarithmic terms. In particular, the charges of 2-branes that wrap a curve $C$ are shifted by $D \cdot C$. Of course this is nothing but a shift of the $B$-fields. It is easy to see, that for large volume transformations with respect to $D=J_{a}$ the brane charges (3.11) transform as $\vec{\Pi} \mapsto M_{a} \cdot \vec{\Pi}$, with

$$
M_{a, I J}=\left(\begin{array}{cccc}
1 & -\delta_{a j} & 0 & 0 \\
0 & \delta_{i j} & -c_{a i j} & c_{a i}^{+} \\
0 & 0 & \delta_{i j} & -\delta_{a i} \\
0 & 0 & 0 & 1
\end{array}\right), \quad c_{a i}^{ \pm}=\frac{1}{2}\left(c_{a a i} \pm c_{a i i}\right)
$$

where $I, J=1, \ldots, 2+2 h^{1,1}$ and $i, j=1, \ldots, h^{1,1}$ while $c_{i j k}$ was defined in (3.12).

Seidel-Thomas twists and conifold transformations. For loops around a component of the discriminant where the Calabi-Yau $M$ itself or a divisor in $M$ collapses to a point, the corresponding transformation of B-branes is a Seidel-Thomas twist [33, 79, 80]. Here 
we will only be interested in the case where $M$ itself collapses which is conjectured to correspond to the principal component of the discriminant [77, 81]. We will denote the corresponding transformation as a conifold transformation or conifold monodromy.

The Fourier-Mukai kernel is given by the ideal sheaf $\mathcal{I}_{\Delta}$ of the diagonal in $M \times M$. In the derived category this is quasi-isomorphic to the complex

$$
0 \rightarrow \mathcal{O}_{M \times M} \rightarrow \mathcal{O}_{\Delta} \rightarrow 0
$$

One can use the Grothendieck-Riemann-Roch theorem (3.15) to translate (3.14) into an action on the Chern characters of branes

$$
\operatorname{ch}\left(\Phi_{\mathcal{E}}\left(\mathcal{F}^{\bullet}\right)\right)=\pi_{1 *}\left(\operatorname{ch}(\mathcal{E}) \cdot \pi_{2}^{*}\left[\operatorname{ch}\left(\mathcal{F}^{\bullet}\right) \operatorname{Td}(M)\right]\right)
$$

Using (3.18) and $\Phi_{\mathcal{O}_{\Delta}}\left(\mathcal{F}^{\bullet}\right)=\mathcal{F}^{\bullet}($ see [82], p. 114) this leads to

$$
\operatorname{ch}\left(\Phi_{\mathcal{I}_{\Delta}}\left(\mathcal{F}^{\bullet}\right)\right)=\operatorname{ch}\left(\mathcal{F}^{\bullet}\right)-\pi_{1 *} \pi_{2}^{*}\left(\operatorname{ch}\left(\mathcal{F}^{\bullet}\right) \operatorname{Td}(M)\right) .
$$

With the definition of the open string index (3.5) the action on the central charges then takes the simple form

$$
\Pi\left(\mathcal{F}^{\bullet}\right) \mapsto \Pi\left(\mathcal{F}^{\bullet}\right)-\chi\left(\mathcal{F}^{\bullet}, \mathcal{O}_{M}\right) \Pi\left(\mathcal{O}_{M}\right)
$$

It is perhaps instructive to give some geometric intuition for this action. Note that multiplication of the charge $\operatorname{ch}\left(\mathcal{F}^{\bullet}\right)$ with the Todd-class $\operatorname{Td}(M)=1+\ldots$ does not change the support of the corresponding brane. Now the effect of the pull-back and push-forward operations in the second term is to make any brane $\mathcal{F}^{\bullet}$ "wrap" the first factor of $M \times M$ and to project out branes that do not have point-like support on the second factor. The only brane that survives this operation is the zero-brane corresponding to a skyscraper sheaf $\mathcal{O}_{p t}$. which is transformed into the structure sheaf $\mathcal{O}_{M}$. Combining this with the first term in (3.21) one can see that the zero brane is transformed into a bound state of a zero brane and an anti six-brane while branes that do not contain embedded zero branes remain unaffected.

Of course, this pictures receives corrections due to the presence of the Todd-class and it is easy to evaluate (3.21) exactly. In particular, we find that our basis of brane charges (3.11) transforms as $\vec{\Pi} \mapsto M_{C} \cdot \vec{\Pi}$, with

$$
M_{C, I J}=\left(\begin{array}{cccc}
1 & 0 & 0 & 0 \\
-\kappa_{i} & \delta_{i j} & 0 & 0 \\
0 & 0 & \delta_{i j} & 0 \\
-1 & 0 & 0 & 1
\end{array}\right), \quad \text { where } \kappa_{i}=\frac{1}{6} c_{i i i}+2 b_{i} .
$$

The definition of $b_{i}$ has been given in (3.12).

\subsection{Relative conifold transformations and $\Gamma_{1}(N)$}

Given a family of complex elliptic curves, the lattice of brane charges is generated by the zero brane $\mathcal{O}_{p t}$. and the two-brane $\mathcal{O}_{C}$. The large volume monodromy $T$ and the conifold 
monodromy $U$ respectively act on the vector of central charges $\vec{\Pi}=\left(\Pi\left(\mathcal{O}_{C}\right), \Pi\left(\mathcal{O}_{p t}\right)\right)^{t}$ as

$$
T: \vec{\Pi} \mapsto\left(\begin{array}{rr}
1 & -1 \\
0 & 1
\end{array}\right) \cdot \vec{\Pi}, \quad U: \vec{\Pi} \mapsto\left(\begin{array}{rr}
0 & 1 \\
-1 & 1
\end{array}\right) \cdot \vec{\Pi} .
$$

Note that the Todd-class is trivial and therefore the geometric intuition that we outlined above applies without further modification. It is easy to see that the corresponding matrices, which we will also denote by $T$ and $U$, generate the modular group $\operatorname{SL}(2, \mathbb{Z})$. In particular, the normalized volume of the curve

$$
\tau=-\frac{\Pi\left(\mathcal{O}_{C}\right)}{\Pi\left(\mathcal{O}_{p t .}\right)},
$$

transforms as

$$
T: \tau \mapsto \tau+1, \quad U: \tau \mapsto \frac{\tau}{1+\tau} .
$$

It turns out that on an elliptic or genus-one fibration this transformation can be performed fiberwise. Perhaps not surprisingly, the relevant Fourier-Mukai kernel is the ideal sheaf of the relative diagonal $\mathcal{I}_{\Delta_{B}}$ in the relative fiber product $M \times_{B} M$ [30]. Following [13] we will therefore call this a relative conifold transformation. Using the singular Riemann-Roch theorem an analogous formula to (3.20) can be derived and reads

$$
\operatorname{ch}\left(\Phi_{\mathcal{I}_{\Delta_{B}}}\left(\mathcal{F}^{\bullet}\right)\right)=\operatorname{ch}\left(\mathcal{F}^{\bullet}\right)-\pi_{1 *} \pi_{2}^{*}\left(\operatorname{ch}\left(\mathcal{F}^{\bullet}\right) \operatorname{Td}_{M / B}\right)
$$

where $\operatorname{Td}_{M / B}$ is the Todd-class of the so-called virtual relative tangent bundle.

To define the latter, one needs a local complete intersection (l.c.i.) morphism $i: M \rightarrow$ $V$ that embeds $M$ into a smooth ambient space $V$ such that $V$ is a bundle $\pi^{\prime}: V \rightarrow B$ over $B$ and $\pi=\pi^{\prime} \circ i$ [83]. When $M$ is a hypersurface or complete intersection in a toric ambient space $V$, the toric ambient space itself often exhibits a compatible toric fibration structure and the inclusion of $M$ is an l.c.i. morphism [13]. In the rest of the paper we will assume this to be the case. The leading behavior of the Todd-class was then found to generically be

$$
\operatorname{Td}_{M / B}=1-\frac{1}{2} c_{1}(B)+\ldots
$$

Let us now assume that $M$ is an elliptic or genus one fibration and parametrize the Kähler form as

$$
\omega=\tau \cdot\left(E_{0}+D\right)+\sum_{i=1}^{r} m_{i} \cdot \sigma\left(E_{i}\right)+\sum_{i=r+1}^{\operatorname{rk}(G)} m_{i} \cdot D_{f, i-r}+\sum_{i=1}^{b_{2}(B)} \tilde{t}_{i} \cdot D_{i}^{\prime} .
$$

where the vertical divisors $D_{i}^{\prime}$ are dual to the curves $C_{i}=\frac{1}{N} E_{0} \cdot D_{i}$ and $D_{i}=\pi^{-1} \tilde{D}_{i}, i=$ $1, \ldots, b_{2}(B)$ is a basis of vertical divisors. Moreover, $E_{i}, i=0, \ldots, r$ are independent $N$-sections and $D$ is a vertical divisor such that $\tilde{E}_{0}=E_{0}+D$ is orthogonal to all of 
these curves. ${ }^{7}$ We will also assume that the fibral divisors $D_{f, i}$ are choosen such that they are orthogonal to the zero-section.

Using the generic behavior of the Todd-class $\operatorname{Td}_{M / B}(3.27)$ one can calculate the action of the relative Conifold transformation $R$ on the Kähler parameters (3.27). The action on the normalized volumes of curves in the base $\tilde{t}_{i}, i=1, \ldots, b_{2}(B)$ is

$$
\begin{aligned}
\tilde{t}_{i} & \mapsto \tilde{t}_{i}+\frac{1}{1+N \tau}\left(\frac{1}{2} \tilde{a}_{i} \tau^{2}+\frac{1}{24} \int_{M} c_{2}(M) D_{i}+\frac{N}{2} a_{i} \tau-\frac{N}{2} m^{a} m^{b} C_{a b}^{i}\right) \\
& =\tilde{t}_{i}+\frac{\tilde{a}_{i}}{2 N} \tau-\frac{\tilde{a}_{i}}{2 N} \frac{\tau}{1+N \tau}+\frac{1}{24} \int_{M} c_{2}(M) D_{i}-\frac{1}{1+N \tau} \cdot \frac{N}{2} m^{a} m^{b} C_{a b}^{i}+A,
\end{aligned}
$$

where

$$
A=\frac{1}{2} \frac{N \tau}{N \tau+1}\left(a_{i}-\frac{1}{12} \int_{M} c_{2}(M) D_{i}\right), \quad \tilde{a}_{i}=\int_{M} \tilde{E}_{0}^{2} \cdot D_{i}, \quad a_{i}=\int_{B} c_{1}(B) D_{i},
$$

and

$$
C_{a b}^{i}=\frac{1}{N} \cdot\left\{\begin{array}{cl}
-\pi_{*}\left(\sigma\left(E_{a}\right) \cdot \sigma\left(E_{b}\right)\right) \cdot C_{\beta} & \text { for } 1 \leq a, b \leq r \\
-\pi_{*}\left(D_{f, a} \cdot D_{f, b}\right) \cdot C_{\beta} & \text { for } r<a, b \leq \operatorname{rk}(G) \\
0 & \text { otherwise }
\end{array}\right.
$$

It was shown in [84] that $A$ vanishes for generic elliptic fibrations. A proof that this result extends at least to the classes of genus one fibrations studied in [85] can be found in appendix B. We can then introduce the shifted Kähler parameters

$$
t_{i}=\tilde{t}_{i}+\frac{\tilde{a}_{i}}{2 N} \tau
$$

and find the action

$$
U:\left\{\begin{array}{rl}
\tau & \mapsto \tau /(1+N \tau) \\
m_{i} & \mapsto m_{i} /(1+N \tau), \quad i=1, \ldots, \operatorname{rk}(G) \\
Q_{i} & \mapsto(-1)^{a_{i}} \exp \left(-\frac{N}{1+N \tau} \cdot \frac{1}{2} m^{a} m^{b} C_{a b}^{i}+\mathcal{O}\left(Q_{i}\right)\right) Q_{i}
\end{array},\right.
$$

where $Q_{i}=\exp \left(2 \pi i t_{i}\right)$ and the action on $Q_{i}$ receives corrections that are double exponentially surpressed in the large base limit. Note that the large volume transformation $T$ that shifts $\tau \mapsto \tau+1$ while leaving $m_{i}, i=1, \ldots, \operatorname{rk}(G)$ invariant acts as

$$
T:\left\{\begin{aligned}
\tau & \mapsto \tau+1 \\
m_{i} & \mapsto m_{i}, \quad i=1, \ldots, \operatorname{rk}(G) \\
Q_{i} & \mapsto(-1)^{\frac{\tilde{a}_{i}}{2 N}} Q_{i}
\end{aligned}\right.
$$

At this point we should review the congruence subgroups $\Gamma_{0}(N)$ and $\Gamma_{1}(N)$ of $\Gamma=$ $\mathrm{SL}(2, \mathbb{Z})$. The Hecke congruence subgroup of level $n$ is defined as

$$
\Gamma_{0}(N)=\left\{\left(\begin{array}{ll}
a & b \\
c & d
\end{array}\right) \in \Gamma: c \equiv 0(\bmod n)\right\},
$$

\footnotetext{
${ }^{7}$ Recall that independence for $N$-sections means that they cannot be related via the action of the Jacobian fibration.
} 
while

$$
\Gamma_{1}(N)=\left\{\left(\begin{array}{ll}
a & b \\
c & d
\end{array}\right) \in \Gamma: a, d \equiv 1(\bmod n), \quad c \equiv 0(\bmod n)\right\},
$$

and it is clear that $\Gamma_{1}(N) \subseteq \Gamma_{0}(N) \subseteq \Gamma$. Both groups have an interesting moduli problem associated to it which will be relevant to us later. Namely, $\Gamma_{0}(N)$ acts on the complex structure parameter $\tau$ of an elliptic curve such that a cyclic subgroup of order $N$ is preserved while $\Gamma_{1}(N)$ preserves also the generator of this group [86].

Let us now discuss generators for $\Gamma_{0}(N)$ and $\Gamma_{1}(N)$ with $N \leq 4$. Using the elements

$$
\tilde{T}=\left(\begin{array}{ll}
1 & 1 \\
0 & 1
\end{array}\right), \quad \tilde{U}_{N}=\left(\begin{array}{cc}
1 & 0 \\
N & 1
\end{array}\right)
$$

we can write $\Gamma_{0}(N)=\left\langle\tilde{T}, \tilde{U}_{N},-\mathbb{1}\right\rangle$ and $\Gamma_{1}(N)=\left\langle\tilde{T}, \tilde{U}_{N}\right\rangle$. Note that the set of generators is not always minimal and in particular one finds $\Gamma_{0}(2)=\Gamma_{1}(2)$. Starting with $N=5$ the generating sets become more complicated and we will restrict ourselves to $N \leq 4$. Because $\Gamma_{0}(N)$ is then obtained from $\Gamma_{1}(N)$ by adjoining the matrix $-\mathbb{1}$ it is clear that the action on $\tau$ and therefore also the rings of modular forms for both groups are identical.

With these definitions we see that for $N \leq 4$ the monodromies $U$ and $T$ generate an action of the congruence subgroup $\Gamma_{1}(N) \subseteq \mathrm{SL}(2, \mathbb{Z})$, or equivalently $\Gamma_{0}(N)$, on the Kähler parameters. Under this action $\tau$ transforms like a modular parameter, $m_{i}$ transform as elliptic parameters and the exponentiated Kähler parameters $Q_{i}$ transform, up to a multiplier system, like lattice Jacobi forms of weight 0 and with index matrix given by $C_{a b}^{i}$.

Note that on the enlarged moduli space, where one does not normalize the 0-brane charge, $U$ and $T$ generate an action of $\Gamma_{1}(N)$ and, for $N>2$, not of $\Gamma_{0}(N)$. In the following we will therefore talk about modularity with respect to $\Gamma_{1}(N)$ although the rings of modular forms do not distinguish between the groups.

\subsection{EZ-transformations and wall monodromies}

The Seidel-Thomas twists admit several generalizations. Horja constructed so-called EZtransformations that arise from loci where a subvariety $E \hookrightarrow M$ collapses onto a subvariety $Z[33,87]$. The conjectured physical interpretation is that there is a whole category of branes becoming massless at these loci. Essentially the massless objects arise from pullbacks of branes on $Z$ and the induced action on any brane $B$ binds a particular subset of massless objects that depends on $B$. The explicit form of the corresponding monodromy action is significantly more complicated than for the Seidel-Thomas twist and we refer to [88] for a discussion that is aimed towards physicists. However, it is clear that the relative conifold transformation should correspond to an EZ-transformation that arises from the monodromy around a locus in the Kähler moduli space where the generic fiber of the fibration collapses to a point.

More generally, EZ-transformations are conjectured to be realized in the A-model as wall monodromies around phase boundaries. For any Calabi-Yau variety that is a fibration we expect that, when all Kähler parameters but the volume $\tau$ of the fiber are deep inside the Kähler cone, the locus $\tau=0$ marks a boundary between the geometric cone and a hybrid 


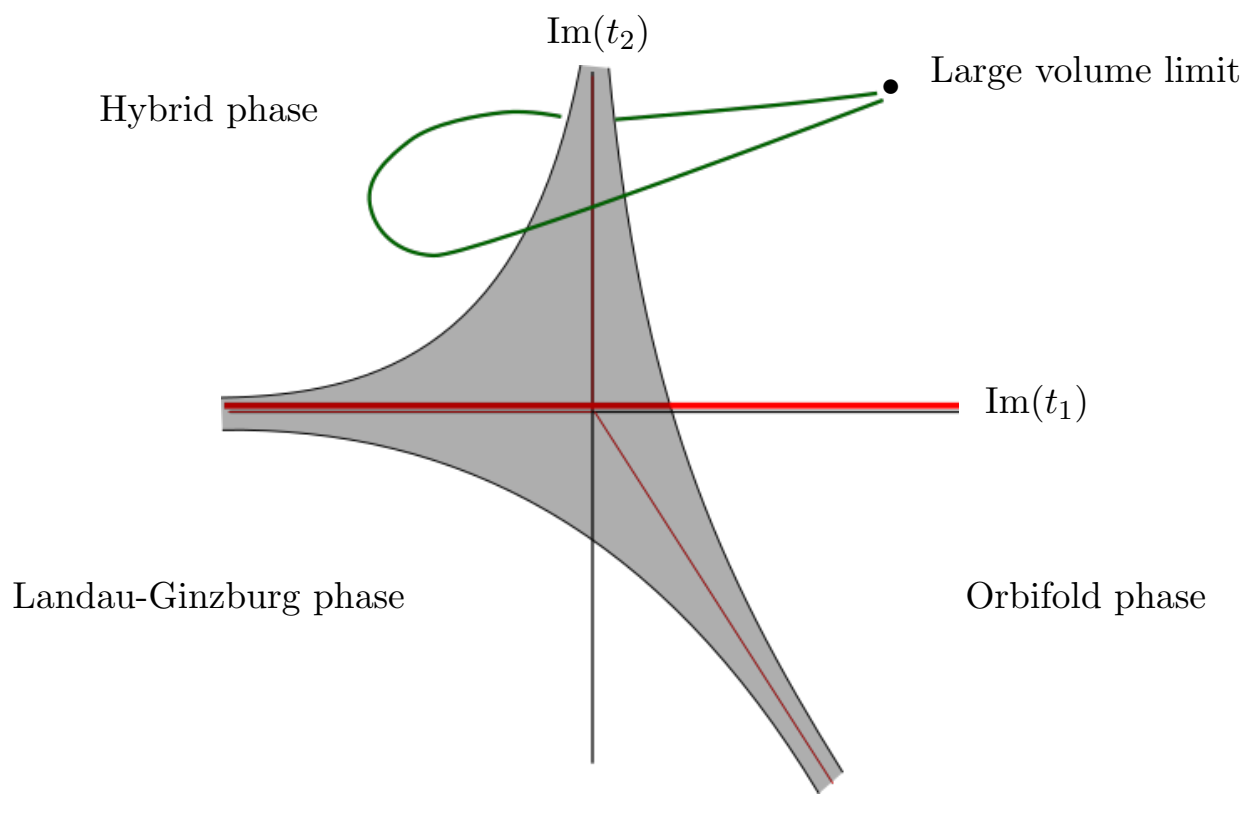

Figure 1. Diagram of the FI-parameter space of the gauged linear sigma model that realizes the non-linear sigma model into the degree 18 hypersurface inside $\mathbb{P}(1,1,1,6,9)$. The amoeba of the principal component of the discriminant, which corresponds to a non-compact Coulomb branch, is indicated in grey. Two geometric phases on the right are seperated by a mixed CoulombHiggs-branch.

phase in the stringy Kähler moduli space. The hybrid phase can be thought of as a LandauGinzberg model that is fibered over a non-linear sigma model. In general, when the volume of the fiber of a fibration vanishes, the category of massless branes is conjectured to be generated by pull-backs of branes in the derived category of the base along the morphism that defines the fibration [88]. One of the branes that becomes massless is therefore in particular the 6-brane that is the pre-image of the base itself. However, the locus in the moduli space where the volume of the 6-brane vanishes is conjectured to correspond to the principal component of the discriminant. The generic monodromy around this locus is just given by a Seidel-Thomas twist with respect to the 6 -brane (3.21). This implies that in the complex structure moduli space of the mirror the point $\tau=0$ with all other Kähler moduli sent to infinity cannot be a normal crossing but corresponds to a tangency between the principal component of the discriminant and the union of the large complex structure divisors. It was shown in [36] (and we review below) that such tangencies imply a relation between the Seidel-Thomas twist with respect to the 6-brane and the large volume monodromies.

We will illustrate these somewhat technical statements with an example. Consider the well-studied Calabi-Yau threefold $X_{18}$ that corresponds to a generic degree 18 hypersurface in the weighted projective space $\mathbb{P}(1,1,1,6,9)$. It is elliptically fibered over the base $\mathbb{P}^{2}$ and the group of divisors is generated by a section $E_{0}$ and one vertical divisor $D_{b}$. A basis of the Kähler cone is generated by $J_{1}=E_{0}+3 D_{b}$ and $J_{2}=D_{b}$ and we expand the Kähler form as

$$
\omega=t^{1} J_{1}+t^{2} J_{2} .
$$


The topological invariants (3.12) are

$$
c_{111}=9, \quad c_{112}=3, \quad c_{122}=1, \quad c_{222}=0, \quad \vec{b}=(17 / 4,3 / 2) .
$$

In Batyrev coordinates the discriminant consists of two components

$$
\Delta_{1}=\left(1-432 z_{1}\right)^{3}-432^{3} \cdot 27 \cdot z_{1}^{3} z_{2}, \quad \Delta_{2}=1+27 z_{2} .
$$

The mirror maps are given by

$$
t_{1}=\frac{1}{2 \pi i} \log \left(z_{1}\right)+\mathcal{O}(z), \quad t_{2}=\frac{1}{2 \pi i} \log \left(z_{2}\right)+\mathcal{O}(z),
$$

where $t_{1}$ is the complexified volume of the generic fiber and $t_{2}$ the complexified volume of a degree one curve in the base. Note that there is a triple tangency between $\Delta_{1}=0$ and $z_{3}=0$ at $z_{1}=1$.

At leading order the complexified Kähler parameters $t_{1}, t_{2}$ can be identified with FI-parameters of a GLSM that realizes the Calabi-Yau as a vacuum manifold. The FIparameter space of such a GLSM is depicted in figure 1. A geometric phase that realizes $X_{18}$ can be found for $\operatorname{Im}\left(t_{1}\right) \gg 0, \operatorname{Im}\left(t_{2}\right) \gg 0$ while there is a hybrid phase around $\operatorname{Im}\left(t_{1}\right) \ll 0, \operatorname{Im}\left(t_{2}\right) \gg 0$. The hybrid phase essentially corresponds to the Landau-Ginzburg model of the elliptic fiber that is fibered over a non-linear sigma model with target space $\mathbb{P}^{2}$. The geometric and the hybrid phase are seperated by a "tentacle" of the amoeba of $\Delta_{1}=0$.

There is a wall monodromy in the A-model that corresponds to a loop around the boundary between the two phases that is taken deep inside the limit of large base volume, i.e. $\operatorname{Im}\left(t_{2}\right) \gg 0$. To obtain the mirror transformation in the B-model we have to move around the discriminant $\Delta_{1}=0$ close to the plane $z_{1}=0$. This has been done for $X_{18}$ in [36] and the procedure is as follows.

One considers a small 3 -sphere $S_{\epsilon}$ around $z_{1}=1, z_{2}=0$. The intersections $L_{1}=$ $S_{\epsilon} \cap\left\{\Delta_{1}=0\right\}$ and $L_{2}=S_{\epsilon} \cap\left\{z_{1}=1\right\}$ are both unknots inside $S_{\epsilon}$ that form a non-trivial link. The shape of this link can be seen directly by taking stereographic coordinates on $S_{\epsilon}$. A plot as well as a schematic depiction of the link is given in figure 2. In the sketch on the right the large volume monodromy corresonds to a loop around the blue line taken outside the link while the generic monodromy around the conifold can be obtained by following a loop around the "handle" of the red line. We now claim that the wall monodromy $M_{\mathrm{W}}$ is related to the generic conifold monodromy $M_{C}$ and to the large base volume monodromy $M_{b}$ is given by

$$
M_{\mathrm{W}}=M_{b}^{-1} \cdot M_{C} \cdot M_{b}^{-1} \cdot M_{C} \cdot M_{b}^{-1} \cdot M_{C} \cdot M_{b}^{3},
$$

while referring the reader to [36] for a detailed discussion of the appropriate paths.

Now how does the wall monodromy $M_{\mathrm{W}}$ act on the brane charges of $X_{18}$ ? Using (3.17) and (3.22) one can immediately calculate the action of $M_{W}$ on our period basis (3.11). It turns out that (3.42) exactly reproduces the action of the Fourier-Mukai transformation that is associated to the ideal sheaf of the relative diagonal. 

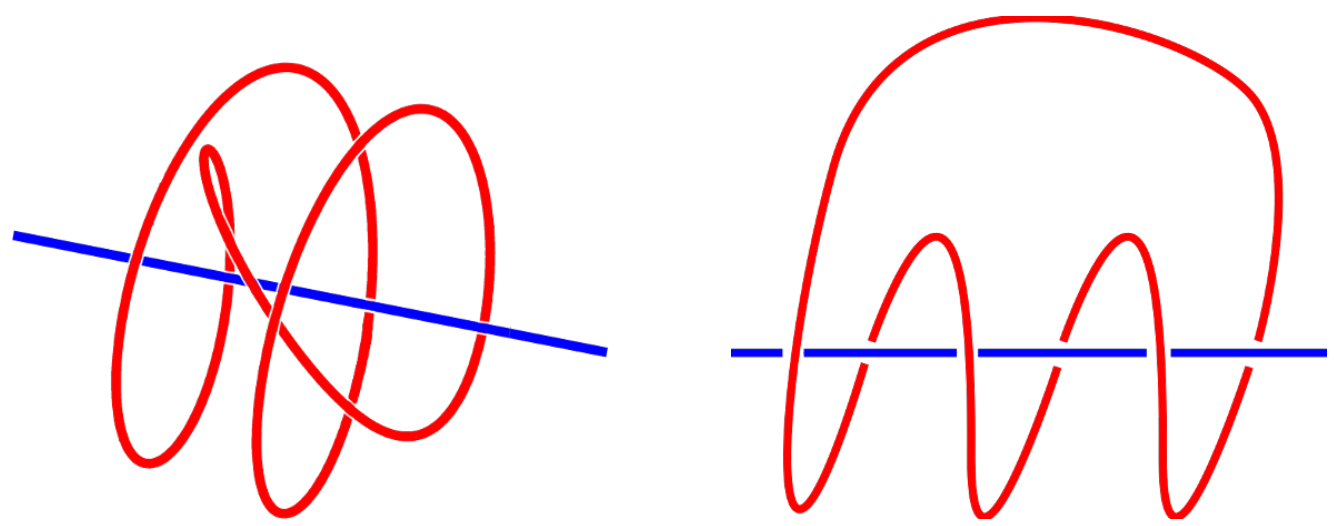

Figure 2. The left image shows a plot of the link that is formed by $\Delta_{1}=0$ (red) and $z_{3}=0$ (blue) inside a small 3 -sphere around the point $z_{1}=1, z_{3}=0$ in the complex structure moduli space of the mirror of $X_{18}$. We use stereographic coordinates on the 3 -sphere and the blue line closes at infinity. The right image shows an equivalent link.

We are now going to show that this relation is generic for any elliptic or genus-one fibration over $\mathbb{P}^{2}$ and derive an analogous relation over Hirzebruch surfaces $\mathbb{F}_{n}$. Let us assume that $J_{b}$ is an effective Cartier divisor on any Calabi-Yau threefold $M$ such that

$$
J_{b}^{3}=0, \quad \text { and } \quad \int_{M} c_{2}(M) \cdot J_{b}=36
$$

We can then use (3.17) and (3.22) to obtain

$$
\begin{aligned}
\left(M_{b}^{-1} \cdot M_{C}\right)^{3} \cdot M_{b}^{3} & =\left(\begin{array}{cccc}
1 & -3 \delta_{b j} & 3 c_{b b j} & 0 \\
-c_{b b i} & x_{2, i j} & x_{3, i j} & 0 \\
0 & -\delta_{b j} \delta_{b i} & \delta_{j i}+c_{b b j} \delta_{b i} & 0 \\
0 & 0 & -c_{b b j} & 1
\end{array}\right), \\
x_{2, i j} & =\left(2 c_{b b i}-c_{b i}^{-}\right) \delta_{b j}+\delta_{j i}, \quad x_{3, i j}=c_{b b j}\left(c_{b i}^{-}-c_{b b i}-\kappa_{i}\right) .
\end{aligned}
$$

This exactly reproduces the action of the $U$-transformation (3.33) on the 2-brane charges of an elliptic or genus-one fibration over $\mathbb{P}^{2}$.

Let us now assume instead that there are two effective Cartier divisors $J_{1}, J_{2}$ and some $n \in \mathbb{N}$ such that

$$
J_{2}^{2}=J_{1}^{3}=J_{1}^{2} J_{2}=J_{1} J_{2}^{2}=0, \quad \int_{M} c_{2}(M) \cdot J_{1}=12(2+n), \quad \int_{M} c_{2}(M) \cdot J_{2}=24 .
$$

This is satisfied when $M$ is an elliptic or genus-one fibration over the Hirzebruch surface $\mathbb{F}_{n}$. More precisely, if we denote the base and the fiber of $\mathbb{F}_{n}$ respectively by $B$ and $F$ then 
$J_{1}=\pi^{-1}(B+F)$ and $J_{2}=\pi^{-1}(F)$. We can then calculate

$$
\begin{aligned}
& \left(M_{1}^{-1} \cdot M_{C} \cdot M_{2}^{-1} \cdot M_{C}\right)^{2} \\
& =\left(\begin{array}{cccc}
1 & n \cdot \delta_{2 j} & c_{11 j}-n \cdot c_{12 j} & 0 \\
-c_{12 i} & y_{22, i j} & y_{23, i j} & 2 c_{1 i}^{-}-c_{2 i i}+4 c_{12 i}+c_{11 i} \\
0 & y_{32, i j} & y_{33, i j} & 2\left(\delta_{1 i}+\delta_{2 i}\right) \\
0 & 0 & -c_{12 j} & 1
\end{array}\right) \text {, } \\
& y_{22, i j}=\delta_{2 j}\left((n-1) c_{12 i}-\frac{n}{2} c_{2 i i}-c_{1 i}^{-}-c_{11 i}\right)+\delta_{1 j}\left(\frac{1}{2} c_{2 i i}-c_{12 i}\right)+\delta_{j i}, \\
& y_{23, i j}=\left(c_{12 j}+c_{11 j}\right)\left(\frac{1}{2} c_{2 i i}-c_{12 i}\right)-\left(c_{1 i}^{-}+\kappa_{i}\right) c_{12 j}+2\left(c_{2 j i}+c_{1 j i}\right), \\
& y_{32, i j}=-\delta_{2 i} \delta_{1 j}-\delta_{1 j} \delta_{2 i}+n \delta_{2 i} \delta_{2 j}, \\
& y_{33, i j}=-\delta_{2 i}\left(c_{12 j}+c_{11 j}\right)-\delta_{1 i} c_{12 j}+\delta_{j i},
\end{aligned}
$$

and find that

$$
M_{W}=\left(M_{1}^{-1} \cdot M_{C} \cdot M_{2}^{-1} \cdot M_{C}\right)^{2} \cdot M_{1}^{2} \cdot M_{2}^{2},
$$

reproduces the action of the $U$-transformation (3.33).

For the sake of completeness let us also discuss the case of a $K 3$ fibration over $\mathbb{P}^{1}$. Assume that there is an effective Cartier divisor $J_{b}$ on any Calabi-Yau threefold $M$ such that

$$
J_{b}^{2}=0, \quad \int_{M} c_{2}(M) \cdot J_{b}=24
$$

Then from $J_{b}^{2}=0$ it follows that $M$ is fibered over $\mathbb{P}^{1}$ and the intersection with $c_{2}(M)$ implies that the generic fiber is a $K 3$ [89]. We calculate

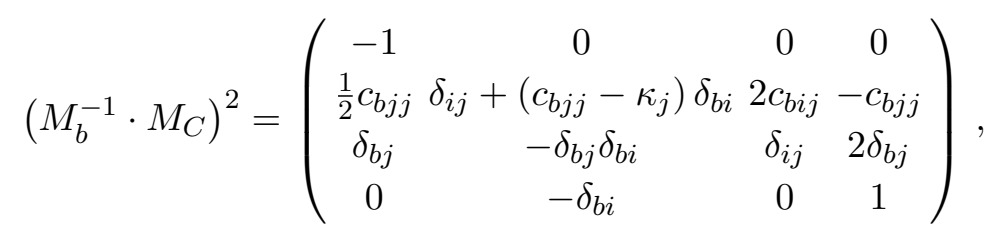

and see that the wall monodromy

$$
M_{W}=\left(M_{b}^{-1} \cdot M_{C}\right)^{2} \cdot M_{b}^{2},
$$

corresponds, in Batyrev coordinates, to a double tangency between the principal component of the discriminant and the large base divisor where the $K 3$ fiber collapses to a point.

The generic form of the monodromies (3.44) and (3.47) suggests that the conditions (3.43) and (3.45) are also sufficient for a Calabi-Yau threefold to be elliptic or genus one fibered respectively over $\mathbb{P}^{2}$ or $\mathbb{F}_{n}$. This can possibly derived from the more general criteria by Oguiso [89] but we are not aware of any previous discussion of these criteria. 
A comment on the swampland. Let us briefly mention that there is a beautiful connection of this story to the swampland distance conjectures, which posit that around loci that are at inifinite distance in the moduli space an infinite tower of states becomes massless. Moreover, according to the emergence conjecture, integrating out the tower of states is what generates the infinite distance in the first place. The swampland distance conjecture in the context of the stringy Kähler moduli space has been discussed in [21, 22, 90-93] and for a recent review of the swampland program see [94]. As we discussed in great detail, the boundary between the hybrid phase and the geometric cone corresponds to a tangency between the discriminant and the large complex structure divisors in the complex structure moduli space of the mirror. This implies that it is at infinite distance and it is natural to ask what the towers of states are that become massless.

The massless states can either correspond to wrapped D-branes or to Kaluza-Klein modes and the wrapped D-branes can become massless on their own or only relative to the Planck scale. The states that arise from wrapped D-branes are called Ramond-Ramond states. At the phase boundaries where the fiber of a fibration collapses we can identify a tower of light Kaluza-Klein modes that arise from the large volume of the base. As we discussed above, the massless branes are supported on the restriction of the fibration to cycles in the base.

On a K3-fibered Calabi-Yau threefold there is only a finite number of Ramond-Ramond states that become massless independently of the Planck scale, because neither multiwrappings of the Calabi-Yau itself nor branes that wrap the fiber over multiple points lead to independent states in the effective theory. In particular, one can consider the multi-scaling limit where the towers from Kaluza-Klein modes and those branes that only become massless relative to the Planck scale decouple, i.e. one sends the Planck scale to infinity. The remaining massless states then yield the $W$ - and $Z$-bosons of an $4 \mathrm{~d} N=2$ gauge theory [95]. When the Planck scale is fixed at a finite value, one expects that NS5branes that wrap the $K 3$ fiber lead to a heterotic string where the tension corresponds to the volume of the fiber [91].

However, for a genus one fibration there is a tower of states from branes that wrap the restriction of the fibration to irreducible curves in the base [96]. For this reason there does not appear to be a well-defined field theory limit when the Planck scale is sent to infinity and when it is kept at a finite value, as expected from 2-fold T-duality, another Type II string emerges [97].

An independent study of the inifinite distance limits from boundaries of the Kähler cone where the fiber of a fibration collapses has been performed in the work [98] that appeared during the final stage of the preparation of this paper.

\subsection{When divisors collapse to curves}

Another example of an EZ-twist arises when a fibral divisor collapses to the curve in the base of the fibration [87]. It is well known that the monodromies around the corresponding locus in Kähler moduli space generate the action of the Weyl group on the Kähler moduli that parametrize the volumes of the components of the familiy of reducible fibers [32, 34, 35]. Let us assume that a rationally fibered fibral divisor $D_{f}$ collapses to a curve of genus $g$ and 
denote the generic fiber of $D_{f}$ by $C$. Then for Calabi-Yau threefolds the corresponding action on brane charges reads [36]

$$
\Pi\left(\mathcal{F}^{\bullet}\right) \mapsto \Pi\left(\mathcal{F}^{\bullet}\right)-\chi\left(\mathcal{O}_{D_{f}}-(1-g) \mathcal{O}_{C}, \mathcal{F}^{\bullet}\right) \cdot \Pi\left(\mathcal{O}_{C}\right)+\chi\left(\mathcal{O}_{C}, \mathcal{F}^{\bullet}\right) \cdot \Pi\left(\mathcal{O}_{D_{f}}\right)
$$

For the examples with fibral divisors that we consider in this paper we verified this formula using matrix factorization techniques in the corresponding gauged linear sigma models $[99,100]$.

\subsection{Monodromies and automorphic properties of $Z_{\text {top }}$}

Having discussed the monodromies that generate the modular transformations of the Kähler moduli we now want to relate this to the modular properties of the topological string partition function $Z_{\mathrm{top}}$.

The automorphic properties under general monodromies can be derived from the wave function interpretation of $Z_{\text {top }}$ [10]. This approach was explored e.g. in [24, 25]. The corresponding Hilbert space arises from quantizing the symplectic vector space $H^{3}(W)$ where $W$ is again the mirror of $M$. Of course, one can equally well consider the symplectic vector space structure on the quantum cohomology ring of $M$. If we choose the real polarization, our positions in this phase space correspond to the central charges of a basis of 0- and 2-branes and the conjugate momenta are central charges of 4- and 6-branes.

Let us first consider the special case that a monodromy does not mix position and momenta or, in other words, it transforms the lattice of 0- and 2-brane charges into itself. In that case the topological string partition function is expected to be invariant under the corresponding action on the flat coordinates. This happens to be the case for some of the monodromies that we discussed above: The large volume monodromies act as $t \mapsto t+1$ on the flat coordinates and the $q$-expansion of $Z_{\text {top }}$ can be interpreted as a consequence. Less trivial are the monodromies that generate the action of the Weyl group. They transform the volumes of fibral curves into each other and $Z_{\text {top }}$ has to be invariant under this action.

We will now show that the elliptic transformation law of the topological string partition function with respect to the geometric elliptic parameters can also be derived in this way. The large volume transformation that shifts $\tau \mapsto \tau+1$ will again be denoted by $T$ and for every volume $m_{i}$ of a rational fibral curve there is a corresponding large volume transformation $M_{i}$ that acts as $m_{i} \mapsto m_{i}+1$. The inverse $U^{-1}$ of the transformation (3.33) acts as

$$
U^{-1}:\left\{\begin{aligned}
\tau & \mapsto \tau /(1-N \tau) \\
m_{i} & \mapsto m_{i} /(1-N \tau), \quad i=1, \ldots, \operatorname{rk}(G) \\
Q_{i} & \mapsto(-1)^{a_{i}} \exp \left(\frac{N}{1-N \tau} \cdot \frac{1}{2} m^{a} m^{b} C_{a b}^{i}+\mathcal{O}\left(Q_{i}\right)\right) Q_{i}
\end{aligned}\right.
$$

where the definitions of $Q_{i}, i=1, \ldots, b_{2}(B), a_{i}$ and $C_{a b}^{i}$ are as in (3.33), (3.30) and (3.31). 
Then the combination $E_{a}=M_{a} \cdot U^{-1} \cdot M_{a}^{-1} \cdot U$ acts as

$$
E_{a}:\left\{\begin{aligned}
\tau & \mapsto \tau \\
m_{i} & \mapsto m_{i}, \quad i=1, \ldots, \operatorname{rk}(G), i \neq a \\
m_{a} & \mapsto m_{a}+N \cdot \tau \\
Q_{i} & \mapsto \exp \left(\frac{N^{2} \cdot \tau}{2} C_{a a}^{i}+N C_{(a b)}^{i} m^{b}\right) Q_{i}
\end{aligned}\right.
$$

Note that (3.53) is exact even away from the large base limit. It is then clear that invariance of $Q^{\beta} Z_{\beta}(\tau, \vec{m}, \lambda)$ under the action of $M_{i}$ and $E_{i}$ implies that

$$
\begin{aligned}
& Z_{\beta}\left(\tau, m_{1}, \ldots, m_{a}+\kappa N \tau+\rho, \ldots, m_{\mathrm{rk}(G)}, \lambda\right) \\
= & \exp \left[-\frac{\beta_{i}}{2}\left(C_{a a}^{i} \kappa^{2} N^{2} \tau+2 C_{(a b)}^{i} N \kappa m^{b}\right)\right] Z_{\beta}(\tau, \vec{m}, \lambda),
\end{aligned}
$$

for all $\kappa, \rho \in \mathbb{Z}$ and $\beta \in H_{2}(B, \mathbb{Z})$. Therefore $Z_{\beta}(\tau, \vec{m}, \lambda)$ satisfies the elliptic transformation law for the geometric elliptic parameters $m_{i}, i=1, \ldots, \operatorname{rk}(G)$ and the index matrix is given by $C_{a b}^{i}$.

It should be possible to make a similar argument that relates the full modular transformation law to the $U$-monodromy, although the mixing of positions and momenta requires a careful treatment of the transformation of $Z_{\text {top }}$. The elliptic transformation law with respect to the topological string coupling constant would then be implied by the modular transformation law. However, this proves to be surprisingly subtle. We will not solve this problem here but to highlight the difficulties it is instructive to review the situation for an elliptic fibration that leads to a trivial gauge group.

As we already discussed above, the Calabi-Yau threefold $M=X_{18}$ is elliptically fibered over $B=\mathbb{P}^{2}$. In particular, following the discussion in 3.3 , we can introduce Kähler parameters $\tau, t$ such that

$$
T:\left\{\begin{array}{l}
\tau \mapsto \tau+1 \\
t \mapsto t+\frac{3}{2}
\end{array}, \quad U:\left\{\begin{array}{l}
\tau \mapsto \frac{\tau}{1+\tau} \\
t \mapsto t+\frac{3}{2}
\end{array},\right.\right.
$$

where in the $U$ transformation we have suppressed terms that are exponentially surpressed in the large base limit. On the other hand, our choice of gauge for the holomorphic 3-form $\Omega$ implies that it transforms like a modular form of weight -1 , i.e.

$$
T: \Omega \mapsto \pm \Omega, \quad U: \Omega \mapsto \frac{ \pm 1}{1+\tau} \Omega
$$

where the sign can be fixed by studying the action of the monodromy in the complex structure moduli space [1]. But $\Omega$ is a section of a line bundle $\mathcal{L}$ on the complex structure moduli space of the mirror and $\lambda$ is a section of $\mathcal{L}$ as well. Therefore the topological string coupling also transforms like a modular form of weight -1 . This is also clear from the action of the monodromies on the enlarged moduli space.

We now want to relate this to the modular properties of the topological string partition function on $X_{18}$. If we consider the string partition function in holomorphic polarization 
it is invariant under monodromies $[24] .^{8}$ The expansion

$$
Z(\tau, t, \lambda)=\exp \left(\sum_{g=0}^{\infty} \lambda^{2 g-2} F_{g}\right),
$$

then implies that the free energies $F_{g}$ transform like modular forms of weight $2 g-2 .^{9}$ It also follows from the discussion in [24] that if we take the limit where the imaginary parts of all Kähler parameters except for $\tau$ go to infinity, then the anholomorphic free energies will be a polynomial in $(\operatorname{Im} \tau)^{-1}$. However, it is easy to see that in this limit the partition function has to remain invariant under the action of $U$ and under the large complex structure monodromies. We can conclude that the anholomorphic free energies $F_{g}$ are almost holomorphic modular forms of weight $2 g-2$.

Note that this implies that the coefficients $Z_{d}(\tau, \lambda)$ in the expansion

$$
Z(\tau, t, \lambda)=Z_{0}(\tau, \lambda)\left(1+\sum_{d=1}^{\infty} Z_{d}(\tau, \lambda) Q^{d}\right)
$$

are, up to a multiplier system, almost holomorphic modular forms of weight 0 . The multiplier system is a consequence of the non-trivial transformation of the base parameter under $U$ and $T$. It turns out that Witten's wave function equation then implies that $Z_{d}(\tau, \lambda)$ is a weak Jacobi form of weight 0 and index $m=d(d-3) / 2[1,101]$. This can be shown as follows.

A weak Jacobi form $\phi_{k, m}(\tau, z)$ (see 4.1 for the definition) can be written as a power series $\phi_{k, m}(\tau, z) \in \widetilde{M}_{\bullet}[[z]]$, where $\widetilde{M}_{\bullet}=\mathbb{C}\left[E_{2}, E_{4}, E_{6}\right]$ is the ring of quasi-modular forms. It is clear that

$$
\tilde{\phi}(\tau, z)=\exp \left(\frac{\pi^{2}}{3} m z^{2} E_{2}(\tau)\right) \phi_{k, m}(\tau, z),
$$

transforms like a weak Jacobi form of index 0 under modular transformations of $\tau$. This implies that $\phi_{k, m}(\tau, z)$ satisfies the differential equation

$$
\left(\frac{\partial}{\partial E_{2}}+\frac{(2 \pi i)^{2}}{12} m z^{2}\right) \phi_{k, m}(\tau, z)=0 .
$$

On the other hand, let us denote the ring of almost holomorphic modular forms by $\widehat{M}_{\bullet}=$ $\mathbb{C}\left[\hat{E}_{2}, E_{4}, E_{6}\right]$ and assume that an element $\hat{f}(\tau, z) \in \widehat{M}_{\bullet}[[z]]$ satisfies the differential equation

$$
\left(\frac{\partial}{\partial \hat{E}_{2}}+\frac{(2 \pi i)^{2}}{12} m z^{2}\right) \hat{f}(\tau, z)=0 \text {. }
$$

Then we know that

$$
\frac{\partial}{\partial \hat{E}_{2}} \exp \left(\frac{\pi^{2}}{3} m z^{2} \hat{E}_{2}(\tau)\right) \hat{f}(\tau, z)=0
$$

\footnotetext{
${ }^{8}$ Possibly up to an overall factor due to the change of gauge which is irrelevant for our discussion.

${ }^{9}$ Following [24] this sum should actually start at genus two. That the genus zero free energy of $X_{18}$ also admits an expansion in terms of quasi-modular forms hints towards the existence of an anholomorphic free energy at genus zero.
} 
and the limit

$$
f(\tau, z)=\lim _{\operatorname{Im} \tau \rightarrow 0} \hat{f}(\tau, z)
$$

satisfies the modular transformation law of a weak Jacobi form.

Let us introduce $z=\frac{\lambda}{2 \pi i}$ where $\lambda$ is again the topological string coupling. The wave function equation for $X_{18}$ then reads in holomorphic polarization [11,68]

$$
\left(\frac{\partial}{\partial \hat{E}_{2}}+\frac{(2 \pi i)^{2}}{12} \frac{d(d-3)}{2} z^{2}\right) Z_{d}(\tau, z)=0 .
$$

In the holomorphic limit $Z_{d}(\tau, z)$ therefore satisfies the modular transformation law of a weak Jacobi form. Furthermore, the Gopakumar-Vafa formula (4.34) implies that the partition function is invariant under shifts $z \rightarrow z+1$ and therefore admits an expansion in terms of $y=\exp (2 \pi i z)$. The elliptic transformation law for shifts of $z$ by $\tau$ follows by combining the modular transformation law and invariance under constant shifts. Assuming validity of the wave function equation we have therefore proven that the coefficients $Z_{d}(\tau, z)$ are weak Jacobi forms of weight 0 and index $d(d-3) / 2$ with a multiplier system. An analogous argument can be made for other elliptic and genus one fibrations that do not exhibit any fibral divisors or additional (multi-)sections. The deriviation of the corresponding modular anomaly equations will be performed in section 4.4.

For elliptic and genus-one fibrations with reducible fibers the exponentiated volumes of curves in the base transform like lattice Jacobi forms with non-trivial index matrix. It is not clear to us how the above argument that relates the relative conifold transformation to the modular properties of the partition function on $X_{18}$ can be generalized. However, it was found that generalizations of the Huang-Katz-Klemm conjecture hold for geometries with fibral divisors [19], multiple sections [21, 22] and also for the refined topological string $[18,19]$. In this paper we study many more examples, including genus-one fibrations with $N$-sections. We find that

$$
Z_{\beta}^{\prime}=Q^{\beta} \cdot Z_{\beta}(\tau, \vec{m}, \lambda),
$$

always transforms like a lattice Jacobi form under $\Gamma_{1}(N)$. The weight is generically zero and the index with respect to the topological string coupling $\lambda$ is $\frac{1}{2} \beta \cdot\left(\beta-c_{1}(B)\right)$. The index matrix of $Z_{\beta}^{\prime}$ with respect to the geometric elliptic parameters $\vec{m}$ is zero.

We conjecture that if the gauge group is fully Higgsable then the topological string partition function is of the form given in (4.29). The ansatz (4.90) for genus-one fibrations with $N$-sections where $N \in\{2,3,4\}$ then follows from the argument that we outline below.

\section{The modular bootstrap for elliptic and genus one fibrations}

In this section we want to generalize the modular bootstrap that has been developed for elliptic fibrations to genus fibered Calabi-Yau threefolds. Based on the results from the previous section it is already clear that instead of modular forms for $\operatorname{SL}(2, \mathbb{Z})$ we will need to consider congruence subgroups $\Gamma_{1}(N)$. We start with a review of modular forms, Jacobi 
forms and the modular bootstrap for elliptic fibrations. By considering Higgs transitions and the corresponding relations among topological string partition functions we are then going to obtain the modular ansatz for genus one fibrations. We will then analyse the base degree zero contributions and find closed expressions for genus one fibrations but also for elliptic fibrations with reducible fibers. Finally, we will study the modular anomaly equations.

\subsection{Rings of modular forms and Jacobi forms for $\Gamma_{1}(N)$}

Much of the following will be well known to the reader. Nevertheless, in the case of modular and Jacobi forms for congruence subgroups some details will be crucial for our discussion and for this reason we do not relegate this section to the appendix.

We recall the definition of the congruence subgroup $\Gamma_{1}(N) \subseteq \mathrm{SL}(2, \mathbb{Z})$ from 3.3

$$
\Gamma_{1}(N)=\left\{\left(\begin{array}{ll}
a & b \\
c & d
\end{array}\right) \in \Gamma: a, d \equiv 1(\bmod n), \quad c \equiv 0(\bmod n)\right\},
$$

and note that $\Gamma_{1}(1)=\mathrm{SL}(2, \mathbb{Z})$. A modular form $f$ of weight $k$ for the congruence subgroup $\Gamma \subset \operatorname{SL}(2, \mathbb{Z})$ is a holomorphic function on the upper half-plane $\mathbb{H}=\{\tau \in \mathbb{C}, \operatorname{Im}(\tau)>0\}$ that is holomorphic at $\tau \rightarrow i \infty$ and satisfies

$$
f\left(\frac{a \tau+b}{c \tau+d}\right)=(c \tau+d)^{k} f(\tau), \quad \text { for }\left(\begin{array}{ll}
a & b \\
c & d
\end{array}\right) \in \Gamma .
$$

We denote the vector space of modular forms of weight $k$ for a group $\Gamma$ by $M_{k}(\Gamma)$ and use the short-hand $M_{k}(N)$ when $\Gamma=\Gamma_{1}(N)$. The corresponding rings of modular forms will be denoted by $M_{*}(\Gamma)$ or $M_{*}(N)$.

The Eisenstein series $E_{2 k}(\tau), k>1$ are modular forms for $\operatorname{SL}(2, \mathbb{Z})$ of weight $2 k$ and can be written as

$$
E_{2 k}(\tau)=1+\frac{2}{\zeta(1-2 k)} \sum_{n=1}^{\infty} \frac{n^{2 k-1} q^{n}}{1-q^{n}} .
$$

For $k=1$ one obtains the quasi modular Eisenstein series $E_{2}(\tau)$ of weight 2 . The Dedekind $\eta$-function

$$
\eta(\tau)=q^{\frac{1}{24}} \prod_{i=1}^{\infty}\left(1-q^{i}\right)
$$

is also not quite modular but satisfies

$$
\eta(\tau+1)=e^{\frac{\pi i}{12}} \eta(\tau), \quad \eta(-1 / \tau)=\sqrt{-i \tau} \eta(\tau) .
$$

However, $\Delta_{12}(\tau)=\eta(\tau)^{24}$ is a modular form of weight 12 for $\operatorname{SL}(2, \mathbb{Z})$ that vanishes as $\tau \rightarrow i \infty$. To generate the rings of modular forms for congruence subgroups $\Gamma_{1}(N)$ let us also introduce

$$
E_{2}^{(N)}(\tau)=-\frac{1}{N-1} \partial_{\tau} \log \left(\frac{\eta(\tau)}{\eta(N \tau)}\right)
$$


which for any $N$ is a modular form for $\Gamma_{0}(N)$ and therefore in particular a modular form for $\Gamma_{1}(N)$. We can then generate the rings of modular forms for $\Gamma_{1}(N)$ and $N \in\{1,2,3,4\}$ as

$$
\begin{aligned}
& M_{*}(1)=\left\langle E_{4}(\tau), E_{6}(\tau)\right\rangle, \\
& M_{*}(2)=\left\langle E_{2}^{(2)}(\tau), E_{4}(\tau)\right\rangle, \\
& M_{*}(3)=\left\langle E_{2}^{(3)}(\tau), E_{4}(\tau), E_{6}(\tau)\right\rangle, \\
& M_{*}(4)=\left\langle E_{2}^{(2)}(\tau), E_{2}^{(4)}(\tau), E_{4}(\tau), E_{6}(\tau)\right\rangle .
\end{aligned}
$$

It is important to note, that for $N=1$ a modular form $f \in M_{k}(1)$ that exhibits a zero of order $n$ at $\tau \rightarrow i \infty$ can be written as

$$
f(\tau)=\Delta_{12}(\tau)^{n} \cdot f^{\prime}(\tau)
$$

where $f^{\prime}(\tau) \in M_{k-12 \cdot n}(1)$ does not vanish at infinity. For $N=2$ the corresponding decomposition of $f \in M_{k}(2)$ is

$$
f(\tau)=\Delta_{4}(\tau)^{n} \cdot f^{\prime}(\tau),
$$

with $f^{\prime} \in M_{k-4 \cdot n}(2)$ and we introduced

$$
\Delta_{4}(\tau)=\frac{\eta(2 \tau)^{16}}{\eta(\tau)^{8}}=\frac{1}{192}\left(E_{4}(\tau)-E_{2}^{(2)}(\tau)^{2}\right) .
$$

Due to the larger set of generators (4.7) an analogous factorization is not possible for a general $f \in M_{*}(N)$ with $N>2$.

The theory of Jacobi forms has been developed in [102] and was extended to multiple elliptic parameters and general finite index subgroups of symplectic groups in [103]. We will only be interested in the following special case. A weak Jacobi form of weight $k$ for $\Gamma_{1}(N) \subseteq \mathrm{SL}(2, \mathbb{Z})$ and with index a symmetric matrix $C \in M_{m \times m}\left(\frac{1}{2} \mathbb{Z}\right)$ is a holomorphic function $\phi(\tau, z) \equiv \phi\left(\tau, z_{1}, \ldots, z_{m}\right)$ on $\mathbb{H} \times \mathbb{C}^{m}$ that satisfies the modular transformation law

$$
\phi\left(\frac{a \tau+b}{c \tau+d}, \frac{z}{c \tau+d}\right)=(c \tau+d)^{k} \exp \left(2 \pi i \frac{c z^{t} C z}{c \tau+d}\right) \phi(\tau, z),
$$

for all $a, b, c, d \in \mathbb{Z}$ with

$$
\left(\begin{array}{ll}
a & b \\
c & d
\end{array}\right) \in \Gamma_{1}(N),
$$

as well as the elliptic transformation law

$$
\phi(\tau, z+\lambda \tau+\mu)=\exp \left(-2 \pi i\left[\lambda^{t} C \lambda \tau+\lambda^{t} C z+z^{t} C \lambda\right]\right) \phi(\tau, z),
$$

for any $\lambda \in \mathbb{Z}^{n}$ and $\mu \in \mathbb{Z}^{n}$. It admits a Fourier expansion

$$
\phi\left(\tau, z_{1}, \ldots, z_{m}\right)=\sum_{n \geq 0} \sum_{r \in \mathbb{Z}^{n}} c(n, r) q^{n} \zeta^{r},
$$

with $q=\exp (2 \pi i \tau)$ and $\zeta^{r}=\exp (2 \pi i z \cdot r)$. 
Of particular importance will be the weak Jacobi forms

$$
\begin{aligned}
\phi_{-2,1}(\tau, z) & =-\frac{\theta_{1}(\tau, z)^{2}}{\eta(\tau)^{6}}=(2 \pi i z)^{2}+\frac{1}{12} E_{2}(\tau)(2 \pi i z)^{4}+\mathcal{O}\left(z^{6}\right), \\
\phi_{0,1}(\tau, z) & =4\left[\frac{\theta_{2}(\tau, z)^{2}}{\theta_{2}(\tau, 0)^{2}}+\frac{\theta_{3}(\tau, z)^{2}}{\theta_{3}(\tau, 0)^{2}}+\frac{\theta_{4}(\tau, z)^{2}}{\theta_{4}(\tau, 0)^{2}}\right]=12+E_{2}(\tau)(2 \pi i z)^{2}+\mathcal{O}\left(z^{4}\right) .
\end{aligned}
$$

of index one and respective weight -2 and 0 . Recall that the Jacobi theta functions are defined as

$$
\begin{aligned}
\theta_{1}(\tau, z) & =\vartheta_{\frac{1}{2} \frac{1}{2}}(\tau, z), \quad \theta_{2}(\tau, z)=\vartheta_{\frac{1}{2} 0}(\tau, z) \\
\theta_{3}(\tau, z) & =\vartheta_{00}(\tau, z), \quad \theta_{4}(\tau, z)=\vartheta_{0 \frac{1}{2}}(\tau, z) \\
\vartheta_{a b}(\tau, z) & =\sum_{n=-\infty}^{\infty} e^{\pi i(n+a)^{2} \tau+2 \pi i z(n+a)+2 \pi i b(n+a)}
\end{aligned}
$$

If we denote the vector space of weak Jacobi forms of weight $k$ and index $C$ for a congruence subgroup $\Gamma$ by $J_{k, C}^{\text {weak }}(\Gamma)$ then

$$
J_{k, m}^{\text {weak }}(\mathrm{SL}(2, \mathbb{Z}))=\oplus_{j=0}^{m} M_{k+2 j}(\mathrm{SL}(2, \mathbb{Z}))\left[\phi_{-2,1}(\tau, z)^{j}, \phi_{0,1}(\tau, z)^{m-j}\right] .
$$

When we discuss the refinement of the topological string partition function over $(-1)$-curves we will also need the Jacobi form

$$
\phi_{-1, \frac{1}{2}}(\tau, z)=i \frac{\theta_{1}(\tau, z)}{\eta(\tau)^{3}}
$$

of weight -1 and index $1 / 2$.

If a weak Jacobi form $\phi(\tau, z)$ for $\Gamma_{1}(N)$ satisfies the stronger condition

$$
\phi\left(\tau, z+\frac{1}{N}\right)=\phi(\tau, z),
$$

i.e. $c(n, r)$ in (4.14) vanishes for $r \notin N \mathbb{Z}$, then the elliptic transformation law follows from the modular transformation law. This can be easily shown from

$$
\begin{aligned}
& \phi(\tau, z+\lambda \tau+\mu)=\phi(\tau, z+\lambda \tau) \\
= & \phi\left(\frac{\tilde{\tau}}{1+N \tilde{\tau}}, \frac{\tilde{z}}{1+N \tilde{\tau}}+\frac{1}{N} \lambda\right), \quad \text { with } \quad \tilde{\tau}=\frac{\tau}{1-N \tau}, \tilde{z}=\frac{z}{1-N \tau}-\frac{1}{N} \lambda
\end{aligned}
$$

A simple example is $\phi(\tau, z)=\phi^{\prime}(N \tau, N z)$, where $\phi^{\prime}(\tau, z)$ is any weak Jacobi form for $\operatorname{SL}(2, \mathbb{Z})$. We will encounter expressions of the form $\phi^{\prime}(N \tau, z)$ that satisfy the elliptic transformation law (4.13) only for $\lambda \in N \mathbb{Z}^{n}$. They satisfy the definition after substituting $z \rightarrow N z$ and in an abuse of language we will also refer to those objects as Jacobi forms for $\Gamma_{1}(N)$. Note that if the index of $\phi^{\prime}(\tau, z)$ is $m$ then the index of $\phi^{\prime}(N \tau, z)$ will be $m / N$.

Another important role will be played by objects of the form

$$
\phi(\tau, z)=\phi^{\prime}\left(N \tau, \tau, z_{1}, \ldots, z_{m-1}\right),
$$


where $\phi^{\prime}$ is again a weak Jacobi form for $\mathrm{SL}(2, \mathbb{Z})$ of weight $k$ and we assume that the index $C$ is block diagonal such that $C_{1 i}=c \cdot \delta_{i, 1}$ for $i=1, \ldots, m$. It is a priori not clear that $\phi(\tau, z)$ in (4.21) is regular at $\tau \rightarrow i \infty$. However, it is easy to see that $q^{c} \phi(\tau, z)$ transforms like a Jacobi form for $\Gamma_{1}(N)$ of weight $k$ and with index matrix $C_{i j}^{\prime}=C_{i+1, k+1} / N$ for $i, j=1, \ldots, m-1$. A special case are Jacobi forms that depend on a single elliptic parameter. In particular, one can check that for any $N$ the functions

$$
A_{N}=\frac{\phi_{0,1}(N \tau, \tau)}{\phi_{-2,1}(N \tau, \tau)}, \quad B_{N}=\frac{1}{q \cdot \phi_{-2,1}(N \tau, \tau)^{N}},
$$

are modular forms of respective weights 2 and $2 N$ under the action of $\Gamma_{1}(N)$ and are holomorphic at $\tau \rightarrow i \infty$. Making an ansatz and comparing a sufficient number of coefficients we then derive the relations

$$
\begin{aligned}
& \phi_{0,1}(2 \tau, \tau)=\frac{E_{2}^{(2)}(\tau)}{\left[q \Delta_{4}(\tau)\right]^{\frac{1}{2}}}, \quad \phi_{-2,1}(2 \tau, \tau)=-\frac{\phi_{0,1}(2 \tau, \tau)}{E_{2}^{(2)}(\tau)}, \\
& \phi_{0,1}(3 \tau, \tau)=\frac{E_{2}^{(3)}(\tau)}{\left[q \Delta_{6}(\tau)\right]^{\frac{1}{3}}}, \quad \phi_{-2,1}(3 \tau, \tau)=-\frac{\phi_{0,1}(3 \tau, \tau)}{E_{2}^{(3)}(\tau)},
\end{aligned}
$$

as well as

$$
\phi_{0,1}(4 \tau, \tau)=\frac{1}{\left[q \Delta_{8}(\tau)\right]^{\frac{1}{4}}} \frac{E_{2}^{(2)}+3 E_{2}^{(4)}}{4}, \quad \phi_{-2,1}(4 \tau, \tau)=-4 \frac{\phi_{0,1}(4 \tau, \tau)}{E_{2}^{(2)}+3 E_{2}^{(4)}},
$$

where we introduced $\Delta_{6} \in M_{6}(3)$ and $\Delta_{8} \in M_{8}(4)$ with

$$
\begin{aligned}
\Delta_{6}(\tau) & =\frac{\eta(3 \tau)^{18}}{\eta(\tau)^{6}}=q^{2}+6 q^{3}+\cdots=\frac{1}{2^{4} \cdot 3^{6}}\left[7\left(E_{2}^{(3)}\right)^{3}-5 E_{2}^{(3)} E_{4}-2 E_{6}\right] \\
\Delta_{8}(\tau) & =\frac{\eta(2 \tau)^{8} \eta(4 \tau)^{16}}{\eta(\tau)^{8}}=q^{3}+8 q^{4}+\ldots \\
& =\frac{1}{2^{17} \cdot 3^{2} \cdot 17}\left[187\left(E_{2}^{(2)}\right)^{4}-144\left(E_{2}^{(4)}\right)^{4}-33 E_{4}^{2}-E_{6}\left(154 E_{2}^{(2)}-144 E_{2}^{(4)}\right)\right] .
\end{aligned}
$$

The denominators of $\phi_{0,1}(N \tau, \tau)$ in (4.23) and (4.24) can be expanded into Eisenstein-like series

$$
\left[q \Delta_{2 N}(\tau)\right]^{\frac{1}{N}}=\frac{1}{\sigma_{1}(N-1)} \sum_{k=1}^{\infty} \sigma_{1}(N \cdot k-1) q^{k},
$$

for $N=2,3,4$ where $\sigma_{k}(d)$ is the divisor function.

\subsection{The modular bootstrap for elliptic fibrations}

Before we consider genus one fibrations let us review the modular bootstrap for elliptic fibrations. The Kähler form can be expanded as

$$
\omega=\tau \cdot\left(E_{0}+c_{1}(B)\right)+\sum_{i=1}^{r} m_{i} \cdot \sigma\left(E_{i}\right)+\sum_{i=r+1}^{\operatorname{rk}(G)} m_{i} \cdot D_{f, i-r}+\sum_{i=1}^{b_{2}(B)} \tilde{t}_{i} \cdot D_{i}^{\prime},
$$


where $E_{0}$ is the class of the zero-section, the divisors $E_{i}, i=1, \ldots, r$ correspond to the sections that generate the Mordell-Weil group, $r$ is the rank of the Mordell-Weil group and $\sigma$ is the Shioda map (A.1). The fibral divisors are denoted by $D_{f, i}, i=1, \ldots, \operatorname{rk}(G)$ and the vertical divisors $D_{i}^{\prime}, i=1, \ldots, b_{2}(B)$ are dual to the curves $C_{i}=E_{0} \cdot D_{i}, i=1, \ldots, b_{2}(B)$. From the discussion in 3.3 it is clear that to see the modular structure we need to introduce shifted Kähler parameters $t_{i}, i=1, \ldots, h^{1,1}(B)$ that are defined as

$$
t_{i}=\tilde{t}_{i}+\frac{\tilde{a}_{i}}{2} \tau, \quad \text { with } \quad \tilde{a}_{i}=\int_{B} c_{1}(B) \cdot \pi\left(D_{i}\right)
$$

We assume that $M$ is an elliptically fibered Calabi-Yau threefold such that on a generic point on the Coulomb branch the gauge group is Abelian. In other words, at a generic point in the complex structure moduli space of $M$ there are no fibral divisors.

Then, if we expand the topological string partition function as in (2.3), the coefficients take the form

$$
Z_{\beta}(\tau, \underline{m}, \lambda)=\frac{1}{\eta(\tau)^{12 \cdot c_{1}(B) \cdot \beta}} \frac{\phi_{\beta}(\tau, \underline{m}, \lambda)}{\prod_{l=1}^{b_{2}(B)} \prod_{s=1}^{\beta_{l}} \phi_{-2,1}(\tau, s \lambda)} .
$$

The numerators $\phi_{\beta}(\tau, \underline{m}, \lambda)$ are Jacobi forms of weight

$$
w=6 c_{1}(B) \cdot \beta-\sum_{l} \beta_{l},
$$

and index

$$
r_{\lambda}^{\beta}=\frac{1}{2} \beta \cdot\left(\beta-c_{1}(B)\right)+\sum_{l} \frac{\beta_{l}\left(\beta_{l}+1\right)\left(2 \beta_{l}+1\right)}{6},
$$

with respect to the elliptic parameter $\lambda$. The index matrix with respect to the geometric elliptic parameters $\underline{m}$ is

$$
r_{i j}^{\beta}=\left\{\begin{array}{cl}
-\frac{1}{2} \pi_{*}\left(\sigma\left(E_{i}\right) \cdot \sigma\left(E_{j}\right)\right) \cdot \beta & \text { for } 1 \leq i, j \leq r \\
-\frac{1}{2} \pi_{*}\left(D_{f, i} \cdot D_{f, j}\right) \cdot \beta & \text { for } r<i, j \leq \operatorname{rk}(G) \\
0 & \text { otherwise }
\end{array}\right.
$$

where $\beta$ is a curve of volume $t_{i}$. Note that

$$
b_{i j}=-\pi_{*}\left(\sigma\left(E_{i}\right) \cdot \sigma\left(E_{j}\right)\right)
$$

is the so-called height pairing of the sections $E_{i}$ and $E_{j}$.

The ansatz (4.29) is based on the results from [1, 19, 21, 22] and we provide additional evidence in this paper. The Dedekind $\eta$-function in the denominator is exactly cancelling the zero at $\tau \rightarrow i \infty$ that comes from $Q^{\beta}$ due to the shift (4.28). Moreover, the product $\prod_{l=1}^{b_{2}(B)} \prod_{s=1}^{\beta_{l}} \phi_{-2,1}(\tau, s \lambda)$ is such that for a given degree the associated Gopakumar-Vafa invariants vanish when the genus is greater than some highest value [1]. Assuming that $Z_{\beta}$ is a meromorphic Jacobi form of the given weight and index therefore seems to imply this ansatz at least modulo some assumptions on the vanishing of enumerative invariants. 
Note that a more complicated form of the denominator occured in [19] but is excluded by our assumption on the Coulomb branch.

It can be further constrained if there are additional dualities for which we know the transformation behaviour of the topological string partition function. This is usually the case when a monodromy does not act on the 0-brane charge and does not transform 2branes into higher dimensional branes. A prime example is the action of the affine Weyl group (see section 3.5) as was first explored in [32]. In the context of the modular bootstrap on non-compact Calabi-Yau that engineer 6d SCFTs, this symmetry was understood as a consequence of properties of elliptic genera [19].

More generally, in the complex structure moduli space of an elliptically or genus one fibered Calabi-Yau $M$ there is a sublocus such that $M$ remains non-singular and the gauge group $G^{\prime}$ associated to $M$ is "maximally non-Abelian". On this locus the vacuum expectation values of all hypermultiplets in the adjoint representation of non-Abelian factors of $G^{\prime}$ are set to zero. The affine Weyl groups of the non-Abelian factors of $G^{\prime}$ act on the geometric elliptic parameters $\underline{m}$ and $Z_{\beta}(\tau, \underline{m}, \lambda)$ is invariant under this action.

\subsection{Closed expressions for $Z_{\beta=0}$}

We will now discuss the base degree zero contributions. For the readers convencience let us recall that the Gopakumar-Vafa formula expresses the sum of the free energies in terms of integer invariants $n_{\beta}^{g}$ via

$$
\log (Z)=\sum_{g=0}^{\infty} \lambda^{2 g-2} F_{g}=\sum_{\beta \in H_{2}(M, \mathbb{Z})} \sum_{g=0}^{\infty} \sum_{m=1}^{\infty} \frac{n_{\beta}^{g}}{m}\left(2 \sin \left(\frac{m \lambda}{2}\right)\right)^{2 g-2} q^{\beta m},
$$

where we have omitted the classical terms. The crucial observation by [1] is that the only non-vanishing Gopakumar-Vafa invariants with degree zero in the base are at genus zero and genus one. All the free energies for $g \geq 2$ are therefore entirely determined by multi-covering contributions from the genus zero curves. The corresponding sum can be evaluated using

$$
\sum_{m=1}^{\infty} \frac{1}{m} \frac{q^{m}}{\left(2 \sin \left(\frac{m \lambda}{2}\right)\right)^{2}}=\lambda^{-2} \cdot \operatorname{Li}_{3}(q)+\sum_{g=1}^{\infty} \lambda^{2 g-2}(-1)^{g+1} \frac{B_{2 g}}{2 g[(2 g-2) !]} \operatorname{Li}_{3-2 g}(q) .
$$

Moreover, the contributions of constant maps are given by

$$
F_{g}^{\text {const }}=(-1)^{g} \chi \frac{B_{2 g} B_{2 g-2}}{4 g(2 g-2)[(2 g-2) !]}, \quad \text { for } \quad g \geq 2 .
$$

An analysis for elliptic fibrations without reducible fibers has been performed in [1] and we will start with a review of this simpler situation. The instanton contribution to the genus zero free energy then takes the form

$$
F_{0}^{\mathrm{inst}}=-\chi \cdot \sum_{i=1}^{\infty} \operatorname{Li}_{3}\left(q^{i}\right)+\mathcal{O}(Q)
$$


where we have used $Q$ to denote Kähler moduli of curves in the base and using

$$
E_{2 g-2}(\tau)=1-\frac{2(2 g-2)}{B_{2 g-2}} \sum_{i=1}^{\infty} \operatorname{Li}_{3-2 g}\left(q^{i}\right)=1+\mathcal{O}(q)
$$

it follows that the higher genus free energies are

$$
F_{g \geq 2}=(-1)^{g} \chi \frac{B_{2 g} B_{2 g-2}}{4 g(2 g-2)(2 g-2) !} E_{2 g-2}(q)+\mathcal{O}(Q) .
$$

The only non-vanishing Gopakumar-Vafa invariants at genus 1 are in multiples of the class of the generic fiber and given by the Euler characteristic of the base $n_{T^{2}}^{1}=\chi_{B}$. However, the corresponding free energy also receives multi-covering contributions (4.35) and reads

$$
F_{1}^{\text {inst }}=\left(\chi_{B}-\frac{\chi}{12}\right) \cdot \sum_{i=1}^{\infty} \operatorname{Li}_{1}\left(q^{i}\right) .
$$

The closed modular expressions for the base degree zero contributions to the higher genus free energies of geometries without reducible fibers rely on the somewhat miraculous interplay between geometric and number theoretic formulas. It is therefore particularly interesting to see how the generalization of these expressions can be derived for fibrations with reducible fibers that lead to additional elliptic parameters.

Geometries with reducible fibers. In our more general setup the only non-vanishing Gopakumar-Vafa invariants for base degree zero still arise at genus zero and genus one. Instead of aiming for full generality we illustrate the situation for geometries with reducible fibers at the example of $M_{2}^{(2)}=\left(F_{6} \rightarrow \mathbb{P}^{2}\right)[\mathrm{U}(1)]_{3}^{-216}$ (see section 7.2.2 for a more detailed discussion of this geometry). The fibration has a holomorphic zero-section $s_{0}$ and a rational section $s_{1}$ that generates the Mordell-Weil group. Here one finds that the base degree zero contribution $F_{0, \beta=0}$ to the genus zero free energy $F_{0}=F_{0, \beta=0}+\mathcal{O}(Q)$ takes the form

$$
\begin{aligned}
F_{0, \beta=0}= & -\chi \cdot \sum_{i=1}^{\infty} \operatorname{Li}_{3}\left(q^{i}\right) \\
& 144 \cdot \operatorname{Li}_{3}(y)+144 \cdot \sum_{i=1}^{\infty}\left(\operatorname{Li}_{3}\left(q^{i} y^{-1}\right)+\operatorname{Li}_{3}\left(q^{i} y\right)\right) \\
& 18 \cdot \operatorname{Li}_{3}\left(y^{2}\right)+18 \cdot \sum_{i=1}^{\infty}\left(\operatorname{Li}_{3}\left(q^{i} y^{-2}\right)+\operatorname{Li}_{3}\left(q^{i} y^{2}\right)\right) .
\end{aligned}
$$

where $q=\exp (2 \pi i \tau), y=\exp (2 \pi i m)$ and $m$ is the volume of the isolated rational fibral curves that lead to charge one hypermultiplets.

More precisely, a contribution of $\operatorname{Li}_{3}(y)$ arises from curves that intersect $s_{1}$ transversely but do not intersect $s_{0}$ while $\operatorname{Li}_{3}\left(y^{2}\right)$ stems from curves $C$ that intersect as $C \cdot s_{1}=-1$ and $C \cdot s_{0}=0$. The $\mathrm{U}(1)$ charge of a fibral curve is counted by the intersection with $s_{1}-s_{0}$ while the Kaluza-Klein charge corresponds to the intersection with $s_{0} \cdot{ }^{10}$ Therefore

\footnotetext{
${ }^{10}$ This statement is slightly modified in the presence of fibral divisors.
} 
the geometry contains 144 curves that lead to matter with $\mathrm{U}(1)$ charge one while another 18 fibral curves lead to hypermultiplets of charge two. Moreover, $\chi=-216$ is the Euler characteristic of $M_{3}^{(2)}$. The multiplicity of matter representations is thus directly encoded in the enumerative invariants of the geometry.

We will now derive closed expressions for the free energies at genus $g \geq 2$. To avoid unnecessary prefactors let us also introduce $y=\exp (z)$. It follows from basic properties of the polylogarithm that

$$
\operatorname{Li}_{s}\left(q^{i} y^{b}\right)=\operatorname{Li}_{s}\left(q^{i} e^{b z}\right)=\sum_{m=0}^{\infty} \frac{z^{m}}{m !} \partial_{z}^{m} \operatorname{Li}_{s}\left(q^{i} e^{b z}\right)=\sum_{m=0}^{\infty} \frac{(b z)^{m}}{m !} \operatorname{Li}_{s-m}\left(q^{i}\right),
$$

and therefore

$$
\sum_{i=1}^{\infty}\left(\operatorname{Li}_{3-2 k}\left(q^{i} y^{-b}\right)+\operatorname{Li}_{3-2 k}\left(q^{i} y^{b}\right)\right)=2 \sum_{i=1}^{\infty} \sum_{m=0}^{\infty} \frac{(b z)^{2 m}}{(2 m) !} \operatorname{Li}_{3-2 k-2 m}\left(q^{i}\right)
$$

We can then use the expansion

$$
\operatorname{Li}_{s}\left(e^{z}\right)=\Gamma(1-s)(-z)^{s-1}+\sum_{k=0}^{\infty} \frac{\zeta(s-k)}{k !} z^{k}
$$

which is valid for integer $s \leq 0$ and $|z|<2 \pi$ as well as the fact that

$$
\zeta(-n)=(-1)^{n} \frac{B_{n+1}}{n+1},
$$

for positive integers $n$ to obtain

$$
\mathrm{Li}_{3-2 k}\left(e^{b z}\right)=(2 k-2) !(b z)^{2-2 k}-\sum_{m=0}^{\infty} \frac{B_{2 m+2 k-2}}{2 m+2 k-2} \frac{(b z)^{2 m}}{(2 m) !}
$$

where we have used that the Bernoulli numbers $B_{n}$ vanish for odd $n>1$. Putting (4.43) and (4.46) together we find that

$$
\begin{aligned}
\Phi_{2 k-2}^{b}(\tau, z) & \equiv \operatorname{Li}_{3-2 k}\left(y^{b}\right)+\sum_{i=1}^{\infty}\left(\operatorname{Li}_{3-2 k}\left(q^{i} y^{-b}\right)+\operatorname{Li}_{3-2 k}\left(q^{i} y^{b}\right)\right) \\
& =(2 k-2) !(b z)^{2-2 k}-\sum_{m=0}^{\infty} \frac{B_{2 m+2 k-2}}{2 m+2 k-2} E_{2 k+2 m-2} \frac{(b z)^{2 m}}{(2 m) !}
\end{aligned}
$$

It is easy to see that $\Phi_{2 k-2}^{b}(\tau, z)$ is a weak Jacobi form of weight $2 k-2$ and index 0 . In total the base degree zero parts of the free energies at genus $g \geq 2$ are therefore given by

$$
F_{g \geq 2, \beta=0}=(-1)^{g} \chi \frac{B_{2 g} B_{2 g-2}}{4 g(2 g-2)(2 g-2) !} E_{2 g-2}(q)-(-1)^{g} \frac{B_{2 g}}{2 g[(2 g-2) !]} \sum_{q=1}^{\infty} n_{q} \Phi_{2 g-2}^{q},
$$

where $n_{q}$ is the number of hypermultiplets of charge $q$. 
Genus-one fibrations with multi-sections. Let us now consider the same problem for fibrations without a section. Again, the only non-vanishing Gopakumar-Vafa invariants for base degree zero arise at genus zero and genus one. We will restrict to the case that the gauge group is $G=\mathbb{Z}_{N}$ which means that there is only one linearly independent $N$-section and there are no fibral divisors.

We start with $N=4$ where the genus zero free energy takes the form

$$
F_{0}=\sum_{i=0}^{\infty}\left[n_{1} \cdot \operatorname{Li}_{3}\left(q^{1+4 i}\right)+n_{2} \cdot \operatorname{Li}_{3}\left(q^{2+4 i}\right)+n_{3} \cdot \operatorname{Li}_{3}\left(q^{3+4 i}\right)+n_{4} \cdot \operatorname{Li}_{3}\left(q^{4+4 i}\right)\right],
$$

where $n_{m}$ is the number of fibral curves that intersect the 4 -section $m$ times. Since the generic fiber intersects the 4 -sections four times it is clear that $n_{1}=n_{3}$ and $n_{4}=-\chi$ where $\chi$ is again the Euler-characteristic of the Calabi-Yau. The expression can therefore be rewritten as

$$
F_{0}=-\chi \cdot \sum_{i=1}^{\infty} \operatorname{Li}_{3}\left(q^{4 i}\right)+n_{1} \cdot \sum_{i=1}^{\infty} \operatorname{Li}_{3}\left(q^{i}\right)+\left(n_{2}-n_{1}\right) \cdot \sum_{i=1}^{\infty} \operatorname{Li}_{3}\left(q^{2 i}\right) .
$$

It is easy to see that for an $N$-section geometry we have multiplicities $n_{i}, i=1, \ldots, N$ and we expect to be able to write

$$
F_{0}=\sum_{m=1}^{N} n_{m} \sum_{i=0}^{\infty} \operatorname{Li}_{3}\left(q^{N \cdot i+m}\right)=\sum_{m=1}^{N} n_{m}^{\prime} \cdot \sum_{i=1}^{\infty} \operatorname{Li}_{3}\left(q^{m i}\right)
$$

where the coefficients $n_{m}^{\prime}, m=1, \ldots, N$ are defined recursively via

$$
n_{m}^{\prime}=n_{m}-\sum_{k<m, k \mid m} n_{k}^{\prime}
$$

However, if we assume that the independent multiplicities are generic, this is only possible when all $0<k<\left\lfloor\frac{N}{2}\right\rfloor$ divide $N$. This is true for the five cases $N=1,2,3,4,6$. For other values of $N$ the identity (4.51) severely constrains the spectrum of charged hypermultiplets. If it can be satisfied we find that

$$
\sum_{m=1}^{N} n_{m}^{\prime}=n_{N}
$$

and it is always true that $n_{N}=-\chi$. We can than use essentially the same argument that worked for elliptic fibrations without reducible fibers to obtain

$$
F_{g \geq 2}=(-1)^{g+1} \frac{B_{2 g} B_{2 g-2}}{4 g(2 g-2)(2 g-2) !} \sum_{m=1}^{N} n_{m}^{\prime} E_{2 g-2}(m \tau)+\mathcal{O}(Q) .
$$

It might be that modularity for general $N$ arises from a more complicated relation between polylogarithms and modular forms for $\Gamma_{1}(N)$. Another possiblity is that in those cases the base degree zero contribution to the higher genus free energies is not modular at all. Perhaps the most exciting, although entirely speculative resolution would be that the identity (4.51) puts a genuine constraint on the curves and intersections in those geometries. 


\subsection{Modular anomaly equations}

In this section our aim is to derive the index $r_{\beta}^{\lambda}$ of the topological string partition function $Z_{\text {top }}$ with respect to the topological string coupling constant $\lambda$. As was explained in section 3.6, this is captured by a modular anomaly equation of the form (3.64).

For ordinary elliptic fibrations, modular anomaly equations have also been derived via duality with elliptic genera of strings and the anomaly inflow mechanism in a $6 \mathrm{~d}$ supergravity theory [18-21]. Here we extend the discussion of $[11,68]$ and argue that whenever $M$ is elliptic or genus one fibered without fibral divisors or additional (multi-)sections, a modular anomaly equation can be derived from the background independence equations introduced in [10]. Strictly speaking, a modification of the background independence equation is necessary that will be discussed in a seperate paper which is written by a different set of authors [2]. For the reader uninterested in technical details we summarize the main result in the paragraph below.

For a Calabi-Yau threefold $M$ that exhibits a genus one fibration with $N$-section such that $h^{1,1}(M)=h^{1,1}(B)+1$, the coefficient $Z_{\beta}(\tau, \lambda)$ in (2.3) satisfies

$$
\left(\frac{\partial}{\partial E_{2}}+\frac{(2 \pi i \lambda)^{2}}{12} r_{\beta}^{\lambda}\right) Z_{\beta}=0,
$$

where

$$
r_{\beta}^{\lambda}=\frac{1}{2 N} \beta \cdot\left(\beta-c_{1}(B)\right) .
$$

This implies that it is a meromorphic Jacobi form for $\Gamma_{1}(N)$ of weight $k=0$ and index $r_{\beta}^{\lambda}$ with respect to the topological string coupling $\lambda$. Since the topological string partition function is independent of complex structure deformations, this derivation also applies to arbitrary genus one fibered Calabi-Yau 3-folds where the gauge group can be completely Higgsed to a discrete group. Together with the derivation of the index matrix with respect to the geometric elliptic parameters we therefore derive all indices directly from properties of the geometry.

Recall that we denote by $W$ the mirror dual of $M$. The topological string partition function $\widehat{Z}{ }^{11}$ is identified with a wave function $\Psi$ on a quantum Hilbert space. The latter is obtained by quantizing the phase space $H_{3}(W, \mathbb{R})$ with symplectic form $\Sigma$ given by its intersection pairing. Quantum background independence of $\widehat{Z}$ on the complex moduli space $\mathcal{M}$ of $W$ leads to the heat equation [10] (see also [24, 25, 104])

$$
\left(\frac{\partial}{\partial t^{\bar{a}}}-\frac{\lambda^{2}}{2} C_{\bar{a}}^{b c} \frac{D}{D t^{a}} \frac{D}{D t^{b}}\right) \widehat{Z}=0 .
$$

Here the coupling $C_{\bar{a}}^{b c}$ is a section of $\mathcal{L}^{-2} \otimes \operatorname{Sym}^{2}\left(T_{\mathcal{M}}^{(1,0)}\right) \otimes T_{\mathcal{M}}^{*(0,1)}$, where $\mathcal{L}$ is the Kähler line bundle on $\mathcal{M}$ with fibers $H^{3,0}(W)$. More precisely, (4.57) was derived as an infinitesimal consequence of the freedom of choice of polarization on $H_{3}(W, \mathbb{R})$ and a change on $\bar{t}^{a}$ acts on the wave function $\hat{Z}$ by a Bogoliubov transformation. A reformulation of the latter

\footnotetext{
${ }^{11}$ We reserve the hat notation for the topological string partition function in holomorphic polarization [24].
} 
transformation is proposed in [2]. However, in the limit case we consider, our result matches with this proposal.

In the following we introduce some quantities, which follow from special geometry over the complex moduli space $\mathcal{M}$ of $W$. The sections of the Kähler line bundle $\mathcal{L}$ are holomorphic three forms $\Omega$ varying holomorphically over $\mathcal{M}$. Moreover, one can choose a symplectic basis in cohomology $\alpha_{I}, \beta^{I} \in H^{3}(W, \mathbb{Z})$ and a dual homology basis $A^{I}, B_{I} \in$ $H_{3}(W, \mathbb{Z}), I=0, \ldots, h^{2,1}(W)$, with pairings $\int_{W} \alpha_{I} \wedge \beta^{J}=-\int_{W} \beta^{J} \wedge \alpha^{I}=\int_{A^{J}} \alpha_{I}=$ $-\int_{B_{I}} \beta^{J}=\delta_{I}^{J}$ and $\int_{A^{J}} \beta^{I}=\int_{B_{I}} \alpha^{J}=0$. Then for a given $z \in \mathcal{M}$ the holomorphic three-form can be expanded as

$$
\Omega(z)=X^{I}(z) \alpha_{I}-F_{I}(z) \beta^{I} .
$$

As a consequence of special geometry one can write the periods as

$$
\vec{\Pi}=\left(\begin{array}{c}
X^{I} \\
F_{I}
\end{array}\right)=X^{0}\left(\begin{array}{c}
1 \\
2 \mathcal{F}^{(0)}-t^{k} \frac{\partial}{\partial t^{k}} \mathcal{F}^{(0)} \\
\frac{\partial}{\partial t^{k}} \mathcal{F}^{(0)}
\end{array}\right),
$$

where $\mathcal{F}^{(0)}$ is the holomorphic prepotential $F(X)=\left(X^{0}\right)^{2} \mathcal{F}^{(0)}(\underline{t})$ and one can introduce the flat coordinates

$$
t^{a}=\frac{X^{a}}{X^{0}}=\frac{1}{2 \pi i} \log \left(z^{a}\right)+\mathcal{O}(\underline{z}) .
$$

The Kähler potential $K$ is related to the holomorphic 3-form $\Omega$ via

$$
e^{-K}=i \int_{W} \Omega \wedge \bar{\Omega}
$$

which can be expressed in terms of the periods as

$$
e^{-K}=i \vec{\Pi}^{\dagger} \Sigma \vec{\Pi}=4\left|X^{0}\right|^{2}\left[\operatorname{Im}\left(\mathcal{F}^{(0)}\right)-t_{2}^{a} \operatorname{Re}\left(\partial_{t^{a}} \mathcal{F}^{(0)}\right)\right] .
$$

Here $t_{2}^{a}:=\operatorname{Im}\left(t^{a}\right)$. A straightforward calculation using (4.61) provides the leading terms

$$
e^{-K}=\frac{4}{3} c_{a b c} t_{2}^{a} t_{2}^{b} t_{2}^{c}+\frac{\zeta(3) \chi(M)}{4 \pi^{3}}+\mathcal{O}(\underline{Q}, \underline{\bar{Q}}),
$$

where $c_{a b c}$ denotes the classical intersection numbers on $M$ and $Q^{a}=\exp \left(2 \pi i t^{a}\right)$. The Weil-Petersson metric follows from the Kähler potential and reads $G_{a \bar{b}}=\partial_{a} \partial_{\bar{b}} K$. Moreover, the coupling $C_{\bar{a}}^{b c}$ is related to the anti-holomorphic Yuakawa coupling $\bar{C}_{\bar{a} \bar{b} \bar{c}} \in \overline{\mathcal{L}}^{2} \otimes$ $\operatorname{Sym}^{3}\left(T_{\mathcal{M}}^{*(0,1)}\right)$, the inversion of the Weil-Petersson metric and the Kähler potential via

$$
C_{\bar{a}}^{b c}=e^{2 K} \bar{C}_{\bar{a} \bar{b} \bar{c}} G^{a \bar{a}} G^{\bar{b} b} .
$$

In the remainder of this section we will assume that $M$ is a genus-one fibered CalabiYau threefold without fibral divisors or additional (multi-)sections. In particular, we use the parametrization of the Kähler form (3.28)

$$
\omega=\tau \cdot\left(\tilde{E}_{0}-\frac{1}{2 N} \sum_{i} \tilde{a}_{i} D_{i}^{\prime}\right)+t^{i} D_{i}^{\prime}
$$


where $\tilde{E}_{0}$ and $D_{i}^{\prime}, i=1, \ldots, h^{1,1}(B)$ have been defined in section 3.3 . The only complexified Kähler moduli are $\tau$ and $t^{i}, i=1, \ldots, h^{1,1}(B)$ where $\tau$ parametrizes the volumes of isolated fibral curves while the shifted Kähler parameters $t^{i}$ parametrize the volumes of curves in the base.

As pointed out in section 3.6 , we are interested in the limit where $\widehat{Z}$ exhibits its anholomorphic behaviour exclusively due to polynomials in $(\operatorname{Im} \tau)^{-1}$. In addition to that, we consider the base parameter $\operatorname{limit} \operatorname{Im} t^{i} \sim \frac{1}{h} \rightarrow \infty$, where $h$ is some real order parameter close to zero, while keeping the fiber parameter $\operatorname{Im} \tau$ finite. We refer to the latter limit as the small fiber limit. ${ }^{12}$ At the end of the day, the quantity of main interest will be the topological string partition function in the holomorphic limit

$$
Z(\tau, \underline{t}, \lambda)=\lim _{\operatorname{Im} \underline{t}, \tau \rightarrow \infty} \widehat{Z}(\tau, \bar{\tau}, \underline{t}, \underline{\bar{t}}, \lambda) .
$$

Let us define

$$
\hat{E}_{0}=\tilde{E}_{0}-\frac{1}{2 N} \sum_{i} \tilde{a}_{i} D_{i}^{\prime}
$$

and denote with $c_{\tau a b}$ the classical intersection matrix given by the intersections $\hat{E}_{0} \cdot J_{a} \cdot J_{b}$ where $J_{a} \in\left\{\hat{E}_{0}, D_{i=1, \ldots, h^{1,1}(B)}^{\prime}\right\}$. We find that

$$
c_{\tau a b}=\left(\begin{array}{c:c}
\alpha & \overrightarrow{0}^{T} \\
\hdashline \overrightarrow{0} & N c_{i j}
\end{array}\right)
$$

Here $c_{i j}=\tilde{D}_{i}^{\prime} \cdot \tilde{D}_{j}^{\prime}$ is the intersection form on the base $B$ with $D_{i}^{\prime}=\pi^{-1} \tilde{D}_{i}^{\prime}$. The $N$ factor arises due to the intersection with the zero- $N$-section. Moreover, $c_{\tau \tau \tau}=\alpha$ is a constant that will drop out from the calculation. With the information obtained from (4.68), (4.63) and taking the appropriate inversions of $G_{a \bar{a}}$, we are able to compute the coupling $C_{\bar{\tau}}^{a b}$ (4.64) in the small fiber limit. Putting everything together, the result reads

$$
C_{\bar{\tau}}^{a b}=\left(\begin{array}{c:c}
\alpha \frac{\tau_{2}^{2}}{V^{2}} h^{4}+\mathcal{O}\left(h^{5}\right) & \frac{1}{6 V^{2} \tau_{2}^{2}}\left(2 \alpha \tau_{2}^{3}-\hat{\chi}\right) N t_{2}^{j} h^{3}+\mathcal{O}\left(h^{5}\right) \\
\hdashline \frac{1}{6 V^{2} \tau_{2}^{2}}\left(2 \alpha \tau_{2}^{3}-\hat{\chi}\right) N t_{2}^{i} h^{3}+\mathcal{O}\left(h^{5}\right) & \frac{1}{4 N \tau_{2}^{2}} c^{\bar{j}}+\mathcal{O}(h)
\end{array}\right)
$$

Here $V=N c_{i j} t^{i} t^{j}, \widehat{\chi}=\frac{\zeta(3) \chi(M)}{4 \pi^{3}}$, and $c^{i j}$ is the inverse of $c_{i j}$.

With this information at our disposal, we proceed applying the small fiber limit on the wavefunction equation (4.57). Denote by $\mathcal{L}_{W}$ the differential operator in (4.57). In the small fiber limit, Witten's wave function equation reduces to

$$
\lim _{h \rightarrow 0} \mathcal{L}_{W}=\frac{\partial}{\partial \hat{E}_{2}}+\frac{\lambda^{2}}{24 N} c^{i j} \frac{\partial}{\partial t^{i}} \frac{\partial}{\partial t^{j}},
$$

where we used the derivative relation $\partial_{\bar{\tau}}=-\frac{3}{2 \pi i \tau_{2}^{2}} \partial_{\hat{E}_{2}}$. Considering the Fourier expansion of $\widehat{Z}$ in the base parameters around the small fiber limit

$$
\lim _{h \rightarrow 0} \widehat{Z}=\widehat{Z}_{0}\left(1+\sum_{\beta \in H_{2}(B, \mathbb{Z})} \widehat{Z}_{\beta}(\tau, \bar{\tau}) Q^{\beta}\right) .
$$

\footnotetext{
${ }^{12}$ Recall from section 4.5 that only in the small fiber limit the exponentiated Kähler parameters of the base transform like Jacobi forms under relative Conifold transformation.
} 
we find that

$$
\lim _{h \rightarrow 0} \mathcal{L}_{W}\left(\widehat{Z}_{0} Q^{\beta}\right)=\left[\frac{1}{2 N}\left(\beta-\frac{c_{1}(B)}{2}\right)^{2}-\frac{1}{5760} \sum_{m=1}^{N} \frac{n_{m}}{N}\right] Q^{\beta} .
$$

The first term on the right hand side of (4.72) comes from the base derivatives in $\mathcal{L}_{W}$ acting on $Q^{\beta-\frac{c_{1}(B)}{2}}$. The shift $c_{1}(B)$ appears due to the classical contributions from $F_{g=1}$ within $\widehat{Z}_{0}$ which read

$$
\left.F_{g=1}\right|_{\text {class }}=-\frac{1}{24} \sum_{a} t^{a} \int_{M} c_{2}(M) \cdot D_{a}^{\prime}=-\frac{1}{2} \sum_{a} t^{a} \int_{B} c_{1}(B) \tilde{D}_{a}^{\prime} .
$$

The second term in (4.72) appears due to the action of $\partial_{\hat{E}_{2}}$ on the base degree zero contribution from $F_{g=2}$ which we derived in the previous section. It can be separated into a purely modular part and a quasi-modular part. The latter reads

$$
\left.F_{g=2, \beta=0}\right|_{\text {quasi }}=-\frac{1}{5760} \sum_{n=1}^{N} \frac{n_{m}}{N} E_{2}(\tau) .
$$

Performing the map $E_{2} \mapsto \hat{E}_{2}$ we recover the non-holomorphic counterpart of (4.74) in $\widehat{Z}_{0}$. Using the pure gravitational anomaly constraints for an effective supergravity in a $6 \mathrm{~d}$ theory, which read

$$
H-V+29 T=273, \quad 9-T=c_{1}^{2}(B),
$$

we are able to verify

$$
\sum_{m=1}^{N} n_{m}=60 c_{1}^{2}(B)
$$

Finally, joining the pieces (4.72) and (4.76) we take the coefficient $Q^{\beta}$ of $\lim _{h \rightarrow 0} \mathcal{L}_{W} \widehat{Z}$. The result reads

$$
\left(\frac{\partial}{\partial \hat{E}_{2}}+\frac{(2 \pi i \lambda)^{2}}{24 N} \beta \cdot\left(\beta-c_{1}(B)\right)\right) \widehat{Z}_{\beta}(\tau, \bar{\tau})=0 .
$$

Taking the holomorphic $\operatorname{limit} \operatorname{Im} \tau \rightarrow \infty$, we obtain (4.55).

\subsection{The modular ansatz for genus one fibrations with $N$-sections}

We will now derive the modular ansatz for the topological string partition function on genus one fibered Calabi-Yau threefolds that do not have a section but only $N$-sections. For reasons that have been described in section 3.3 we restrict ourselves to $N \in\{2,3,4\}$. Then our analysis of the monodromies in the stringy Kähler moduli space led to the conjecture that $Q^{\beta} Z_{\beta}$ transforms like a weak Jacobi form of weight zero and index $\frac{1}{2 N} \beta \cdot\left(\beta-c_{1}(B)\right)$ under the action of $\Gamma_{1}(N)$.

We could now try to derive the denominator of the ansatz following the argument for elliptic fibrations to justify (4.29). However, this is complicated by the fact that the rings of modular forms for $\Gamma_{1}(N)$ in general contain multiple irreducible elements that vanish 

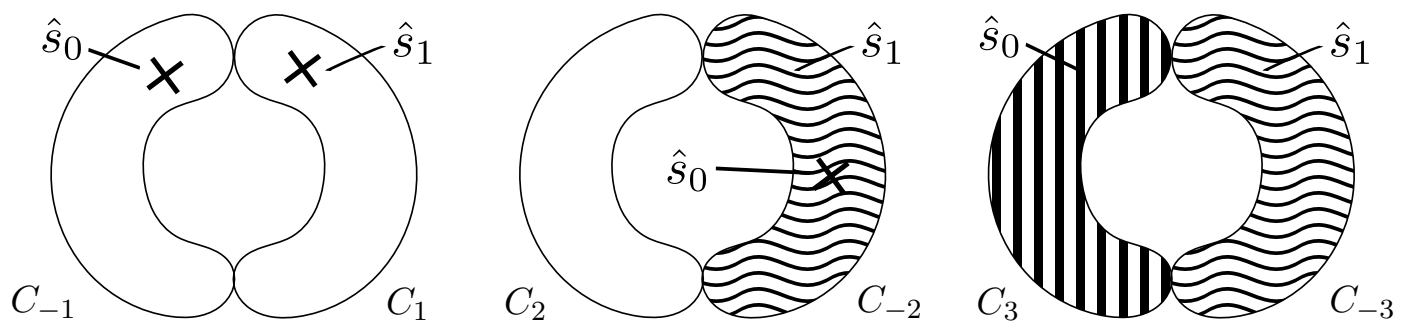

Figure 3. Intersections between the two rational sections and the components of the three types of isolated fibral curves in fibrations constructed from $d P_{1}$ (polytope $F_{3}$ ). Shaded components are wrapped by the rational section while crosses indicate a transverse intersection. The figures are taken from [85].

at $\tau \rightarrow i \infty$. Let us therefore follow a different route and derive the ansatz by considering Higgs transitions.

We will first recall the geometric transition that corresponds to Higgsing an Abelian gauge group into a discrete gauge group within F-theory. This will allow us to identify a good set of Kähler parameters on the multi-section geometry and we see that the relative conifold monodromy and a large volume monodromy generate an action of a congruence subgroup $\Gamma_{1}(N)$ of $\operatorname{PSL}(2, \mathbb{Z})$ on the moduli. We will then use the Higgs transition and the previously conjectured modular properties of the topological string partition function on geometries with multiple sections to show that the topological string partition function on fibrations that only have multi-sections can be expressed in terms of Jacobi forms for $\Gamma_{1}(N)$.

It is well known that via F-theory multi-section geometries lead to discrete gauge symmetries. Moreover, general arguments of quantum gravity imply that discrete gauge symmetries are always the remnant of a Higgsed continuous gauge symmetry. It is therefore expected that for every multi-section geometry $X^{\prime}$ there exists a geometry $X$ with multiple sections such that $X^{\prime}$ can be obtained from $X$ via a conifold transition. More precisely, the geometric transition relates the five dimensional theories that are obtained after compactifying the F-theory vacua on a circle. First the volume of an isolated fibral curve shrinks to zero such that the corresponding hypermultiplets of $\mathrm{U}(1)$ charge $q$ become massless. Note that the volumes of fibral curves correspond to gauge fluxes along the $S^{1}$. A subsequent complex structure deformation can be interpreted as giving a vacuum expectation value to the massless scalar fields in the hypermultiplets.

To be concrete let us consider a generic fibration $X$ that can be obtained from fibers in $d P_{1}$. There are two independent rational sections $s_{0}$ and $s_{1}$ and we can choose $s_{0}$ as the zero-section [85]. The generic gauge group is $G=\mathrm{U}(1)$ which reflects the fact that there are no fibral divisors. There are three types of isolated fibral curves and the intersections with $s_{0}$ and $s_{1}$ are indicated in figure 3 . Note that shaded components are wrapped by the rational section while crosses indicate a transverse intersection. Recall that up to vertical divisors the image of $s_{1}$ under the Shioda map is given by

$$
\sigma\left(s_{1}\right)=E_{1}-E_{0}+(\text { vertical divisors }),
$$

where $E_{0}$ and $E_{1}$ are the divisor classes of $s_{0}$ and $s_{1}$. For a curve $C$ that is wrapped by $s_{i}$ 
the intersection is $E_{i} \cdot C=-1$. Together with the fact that an M2-brane that wraps an isolated fibral curve $C$ has $\mathrm{U}(1)$ charge

$$
q=\sigma\left(s_{1}\right) \cdot C,
$$

this leads to the corresponding labels $C_{q}$ in figure 3 .

If we now try to Higgs the $\mathrm{U}(1)$ with a scalar of charge $q= \pm 3$ we have to let the volume of $C_{3}$ or $C_{-3}$ shrink to zero. We parametrize the Kähler form as in (3.28). Then $\tau$ is the volume of the generic fiber while $m$ is the volume of $C_{1}$ while $t_{i}, i=1, \ldots, h^{1,1}(B)$ are parametrizing the size of curves in the base. In particular, the sizes of $C_{3}$ and $C_{-3}$ are

$$
\operatorname{vol}\left(C_{3}\right)=3 m-\tau, \quad \operatorname{vol}\left(C_{-3}\right)=2 \tau-3 m
$$

It turns out that if we choose $\tau$ and $m \neq 0$ such that $2 \tau-3 m=0$, the Kähler form $\omega$ is always outside the closure of the Kähler cone. However, if $m>0$ the limit $\tau \rightarrow 3 m$ is on the boundary of the Kähler cone. It is then possible to deform the complex structure such that the sections $s_{i}$ merge with two-sections into two three-sections. The corresponding divisor classes are equivalent up to vertical divisors.

In other words, the topological string partition function on the three-section geometry $X^{\prime}$ is obtained from the partition function on the geometry with two sections $X$ by substituting

$$
\tau \mapsto 3 \tau, \quad m \mapsto \tau .
$$

Using the ansatz (4.29) and the relations (4.23) we find that at base degree $\beta$ it takes the form

$$
Z_{\beta}^{X^{\prime}}(\tau, \lambda)=\frac{1}{\eta(3 \tau)^{12 c_{1}(B) \cdot \beta} \Delta_{6}(\tau)^{\frac{r_{\beta}}{3}}} \frac{\phi_{\beta}^{\prime}(\tau, \lambda)}{\prod_{l=1}^{b_{2}(B)} \prod_{s=1}^{\beta_{l}} \phi_{-2,1}(3 \tau, s \lambda)},
$$

where $\phi_{\beta}^{\prime}(\tau, \lambda)$ is a weak Jacobi form for $\Gamma_{1}(3)$ of weight and index such that $Z_{\beta}^{X^{\prime}}(\tau, \lambda)$ is a meromorphic Jacobi form of weight zero and index $\frac{1}{6} \beta \cdot\left(\beta-c_{1}(B)\right)$. Recall that $r_{\beta}=\frac{1}{2} b \cdot \beta$ is the index of $Z^{X}$ with respect to $m$ where $b$ is the height pairing of the section $E_{1}$ with itself.

Let us denote the class of the three-section in the resulting smooth genus-one fibration $X^{\prime}$ by $E^{\prime}$. From a geometric perspective the result from the previous analysis is that there exists a fibral curve $C$ such that $E^{\prime} \cdot C=1$. Therefore, in order to calculate enumerative invariants, we should parametrize the Kähler form as

$$
\omega=\tau \cdot\left(E^{\prime}+D\right)+\ldots,
$$

where $\tau$ is now one third of the volume of the generic fiber and $D$ is vertical divisor such that $\tilde{E}=E^{\prime}+D$ is orthogonal to a basis of curves in the base. In general, a curve that intersects the three-section once could be absent but the ansatz remains valid.

An analogous ansatz holds for two- and four-section geometries. But before we give the expressions, let us note that in every example we find that the factor of $\Delta_{2 N}^{r_{\beta} / N}$ in the 
denominator is compensated by a factor $\Delta_{2 N}^{\left\lceil r_{\beta} / N\right\rceil}$ in the numerator. Cancellation of poles then implies the congruence relation

$$
1-\frac{r_{\beta}}{N} \equiv \frac{1}{2}\left[N c_{1}(B)-\frac{\tilde{a}}{N}\right] \cdot \beta \bmod 1 .
$$

In particular, for a genus one fibration with $N$-sections where $N=2,3,4$ that does not have additional fibral divisors or independent multi-sections we find the simplified ansatz

$$
Z_{\beta}^{(N)}(\tau, \lambda)=\frac{1}{\eta(N \tau)^{12 c_{1}(B) \cdot \beta}} \frac{\phi_{\beta}^{\prime}(\tau, \lambda)}{\prod_{l=1}^{b_{2}(B)} \prod_{s=1}^{\beta_{l}} \phi_{-2,1}(N \tau, s \lambda)},
$$

where the numerator $\phi_{\beta}^{\prime}(\tau, \lambda)$ is an element

$$
\phi_{\beta}^{\prime}(\tau, \lambda) \in M_{*}(N)\left[\phi_{-2,1}(N \tau, \lambda), \phi_{0,1}(N \tau, \lambda)\right] \cdot \Delta_{2 N}(\tau)^{1-\frac{r_{\beta}}{N} \bmod 1},
$$

such that the overall weight of $Z_{\beta}$ is zero and the index is $\frac{1}{2 N} \beta \cdot\left(\beta-c_{1}(B)\right)$. The rings $M_{*}(N)$ are defined in (4.7) and the exponent of $\Delta_{2 N}(\tau)$ can be obtained from (4.92).

\subsection{Summary of the modular bootstrap ansatz}

We will now summarize the ansatz for a genus one fibered Calabi-Yau threefold $M$ with $N$-sections for $N \in\{2,3,4\}$. The Kähler form should be expanded as

$$
\omega=\tau \cdot\left(E_{0}+D\right)+\sum_{i=1}^{r} m_{i} \cdot \sigma\left(E_{i}\right)+\sum_{i=r+1}^{\mathrm{rk}(G)} m_{i} \cdot D_{f, i-r}+\sum_{i=1}^{b_{2}(B)} \tilde{t}_{i} \cdot D_{i}^{\prime},
$$

where a basis of fibral divisors is denoted by $D_{f, i}, i=1, \ldots, \operatorname{rk}(G)$ and $E_{i}, i=0, \ldots, r$ are independent $N$-sections. The vertical divisors $D_{i}^{\prime}, i=1, \ldots, b_{2}(B)$ are dual to the curves $C_{i}=E_{0} \cdot D_{i}, i=1, \ldots, b_{2}(B)$ with $D_{i}=\pi^{-1} \tilde{D}_{i}$ in the sense that

$$
D_{i}^{\prime} \cdot C_{j}=N \cdot \delta_{i j}
$$

The "zero $N$-section" $E_{0}$ is shifted by the unique vertical divisor $D$ such that $\tilde{E}_{0}=E_{0}+D$ is orthogonal to all curves $C_{i}, i=1, \ldots, b_{2}(B)$. Moreover, following the discussion in section 3.3, the shifted Kähler parameters $t_{i}, i=1, \ldots, h^{1,1}(B)$ are defined as

$$
t_{i}=\tilde{t}_{i}+\frac{\tilde{a}_{i}}{2 N} \tau, \quad \text { with } \quad \tilde{a}_{i}=\int_{M} \tilde{E}_{0}^{2} \cdot D_{i} .
$$

We will also assume that there are no fibral divisors at a generic point of the complex structure moduli space of $M$.

Then, if we expand the topological string partition function as in (2.3), the coefficients take the form

$$
Z_{\beta}(\tau, \underline{m}, \lambda)=\frac{1}{\eta(N \tau)^{12 \cdot c_{1}(B) \cdot \beta}} \frac{\phi_{\beta}(\tau, \underline{m}, \lambda)}{\prod_{l=1}^{b_{2}(B)} \prod_{s=1}^{\beta_{l}} \phi_{-2,1}(N \tau, s \lambda)},
$$


where the numerator $\phi_{\beta}(\tau, \lambda)$ is an element

$$
\phi_{\beta}(\tau, \underline{m}, \lambda) \in M_{*}(N)\left[\phi_{-2,1}(N \tau, \bullet), \phi_{0,1}(N \tau, \bullet)\right] \cdot \Delta_{2 N}(\tau)^{1-\frac{r_{\beta}}{N} \bmod 1},
$$

where $\bullet$ stands for any elliptic parameter $z \in\{\lambda, \underline{m}\}$. The exponent of $\Delta_{2 N}(\tau)$ is determined by the congruence relation

$$
1-\frac{r_{\beta}}{N} \equiv \frac{1}{2}\left[N c_{1}(B)-\frac{\tilde{a}}{N}\right] \cdot \beta \bmod 1
$$

The weight of $\phi_{\beta}$ is given in (4.30), while the index with respect to the topological string coupling $\lambda$ is

$$
r_{\beta}^{\lambda}=\frac{1}{2 N} \beta \cdot\left(\beta-c_{1}(B)\right)
$$

The index matrix with respect to the geometric elliptic parameters $m_{i}, i=1, \ldots, \operatorname{rk}(G)$ is

$$
r_{i j}^{\beta}=\frac{1}{N} \cdot\left\{\begin{array}{cl}
-\frac{1}{2} \pi_{*}\left(\sigma\left(E_{i}\right) \cdot \sigma\left(E_{j}\right)\right) \cdot \beta & \text { for } 1 \leq i, j \leq r \\
-\frac{1}{2} \pi_{*}\left(D_{f, i} \cdot D_{f, j}\right) \cdot \beta & \text { for } r<i, j \leq \operatorname{rk}(G) \\
0 & \text { otherwise }
\end{array}\right.
$$

\section{Duality with heterotic strings on CHL orbifolds}

In this section we will argue that heterotic strings on CHL orbifolds $\left(K 3 \times T^{2}\right) / \mathbb{Z}_{N}$ are dual to Type IIA strings on genus one fibered Calabi-Yau threefolds with $N$-sections. We will then systematically construct Calabi-Yaus that are dual to heterotic compactifications on $\left(K 3 \times T^{2}\right) / \mathbb{Z}_{2}$. The modular bootstrap for multi-section geometries that we developed in the previous sections allows us to compare the topological string partition function against the heterotic one-loop computations that have been performed in [51]. This provides an all-genus check of our proposal.

\subsection{Heterotic/Type IIA duality and topological strings}

It is well known that heterotic $E_{8} \times E_{8}$ string theory on $K 3 \times T^{2}$ is dual to Type II strings on Calabi-Yau manifolds [105]. Let us further assume that the dual heterotic theory is weakly coupled in the geometric regime of the moduli space of the Type II compactification and that the $T^{2}$ can be decompactified. Then one can show that the Calabi-Yau has to be a fibration of elliptic $K 3$ surfaces over $\mathbb{P}^{1}$ [106]. Of course, in order to specify a heterotic vacuum one also needs to choose a gauge background on the compactification space. The unbroken gauge symmetry, the spectrum and therefore also the dual Calabi-Yau depend on this choice of background.

An important observable that can be matched for a given dual pair is the gravitational coupling $F_{g}(t, \bar{t})$, which enters via the terms

$$
S=\int \tilde{F}_{g}(t, \bar{t}) F^{2 g-2} R^{2},
$$


into the $d=4, N=2$ effective action. Here $F, R$ are the self-dual parts of the graviphoton and the Riemann curvature. On the heterotic side, all of these couplings are perturbatively one-loop exact but receive non-perturbative corrections. From the Type IIA perspective, $\tilde{F}_{g}$ is a $g$-loop correction and corresponds to the topological string free energy at genus $g[52,53]$. More precisely, the holomorphic limit of the topological string free energies corresponds to

$$
F_{g}^{\mathrm{top}}(t)=\frac{1}{2(2 \pi i)^{2 g-2}} \bar{F}_{g}^{\mathrm{hol}}(t), \quad \text { with } \quad F_{g}^{\mathrm{hol}}(\bar{t})=\lim _{t \rightarrow \infty} \tilde{F}_{g}(t, \bar{t}) .
$$

The heterotic dilaton can be identified with the complexified volume of the $\mathbb{P}^{1}$ that is the base of the $K 3$ fibration in the dual Type II compactification. The complex structure and the complexified volume of the $T^{2}$ on the heterotic side correspond to linear combinations of the complexified volumes of the elliptic fiber and the $\mathbb{P}^{1}$ base of the $K 3$ fiber. Wilson lines on the heterotic torus can be matched with volumes of fibral curves in the elliptic fibration.

For particular choices of bundles the one-loop calculation on the heterotic side has been carried out explicitly $[53,54,107]$. The calculation involves an integral of a product of modular forms and theta functions over the fundamental domain of $\operatorname{PSL}(2, \mathbb{Z})$. This has been evaluated [54] using the lattice reduction theorem by Borcherds [108]. The modular form that appears in the integrand is closely related to the new supersymmetric index [109]

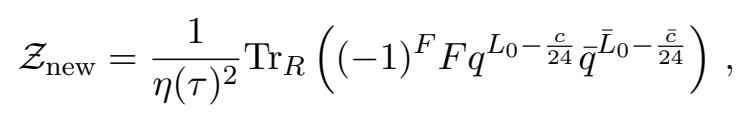

which can be expressed in terms of the elliptic genus of $K 3$ [110]. A variant of moonshine relates the latter to representations of the Mathieu group $M_{24}$ [111] and the consequences for compactifications on $K 3 \times T^{2}$ were studied by [112].

The corresponding dual Calabi-Yau manifolds are $K 3$-fibered and this makes it possible to obtain all-genus results for the topological string at least for a restricted set of curve classes [113]. This relies on the fact that genus $g$ curves on elliptically fibered $K 3$ can be counted by focusing on certain degenerate configurations where $g$-tori are glued to points of the base $\mathbb{P}^{1}[114]$. A resolution of the moduli space of such curves together with a choice of bundle is then given by the Hilbert scheme of $g$ points on $K 3$ and the enumerative invariant is the corresponding Euler characteristic. The result for $K 3$ can be lifted to obtain product formulas that capture all-genus invariants of $K 3 \times T^{2}$ [115] which in turn admit generalizations to regular $K 3$ fibrations [113]. The results from both calculations match which provides highly non-trivial evidence for the duality [113].

\subsection{Genus one fibrations and heterotic strings on $\left(K 3 \times T^{2}\right) / \mathbb{Z}_{N}$}

Soon after heterotic/Type II duality had been established for $K 3 \times T^{2}$ compactifications the arguments have been extended to heterotic strings on so-called CHL quotients $(K 3 \times$ $\left.T^{2}\right) / \mathbb{Z}_{2}$ [39]. A dual geometry was constructed by taking an elliptically fibered Calabi-Yau with a torsional section and taking a $\mathbb{Z}_{2}$ quotient that involves the corresponding shift along the fiber. The result is genus one fibered Calabi-Yau threefold with 2-sections.

However, a detailed discussion of heterotic CHL compactifications on $\left(K 3 \times Z^{2}\right) / \mathbb{Z}_{N}$ only appeared much later [40]. Non-standard embeddings of the gauge connection for CHL 
orbifolds have subsequently been considered in [50] and also the one-loop calculation [54] has been generalized, which led to all-genus predictions for the enumerative invariants of dual Calabi-Yau geometries [51]. It turns out that the new supersymmetric index and therefore also the integrand in the one-loop calculation can be expressed in terms of modular forms for $\Gamma_{1}(N)$.

For CHL quotients of order 2 such that the element $g^{\prime} \in M_{23}$ corresponds to the conjugacy class $2 A$ of $M_{24}$ it was found [50] that the new supersymmetric index only depends on the number of vector- and hypermultiplets in the spectrum. In fact, only the conveniently normalized combination

$$
\hat{b}=\frac{1}{144}\left(N_{h}-N_{v}+12\right),
$$

appears to be relevant [50]. Note that the Hodge numbers of the dual Calabi-Yau are related to the number of vector- and hypermultiplets via

$$
h^{1,1}=N_{v}-1, \quad h^{2,1}=N_{h}-1 .
$$

Two candidate Calabi-Yau duals for $N=2$ CHL compactifications with $\hat{b}=\frac{2}{3}$ and $\hat{b}=\frac{8}{9}$ have been identified by explicitly comparing the predictions from [51] to the genus zero enumerative invariants of Calabi-Yau threefolds with $h^{1,1}=3$ [116]. We will show below that those geometries are again genus one fibered with 2-sections and, using the modular bootstrap, extend the comparison to all genera.

The action of the $\mathbb{Z}_{N}$ quotient on $K 3 \times T^{2}$ is as follows. It was shown by Mukai [46] that the automorphisms of a $K 3$ surface form a subgroup of the Mathieu group $M_{23}$ which is in turn a subgroup $M_{23} \subset M_{24}$. A smooth CHL orbifold is therefore obtained from an order $N$ element $g^{\prime} \in M_{23}$ that acts together with a $1 / N$ shift along a cycle of $T^{2}$. Other quotients are possible at the level of the CFT $[47,117]$ but they do not admit a straightforward geometric interpretation. This is of course closely related to the moonshine phenomenon that we alluded to in the previous section.

At this point we can already argue on general grounds that $\left(K 3 \times T^{2}\right) / \mathbb{Z}_{N}$ should be dual to Type IIA on $N$-section geometries. Following the usual convention we denote the complex structure and the complexified Kähler modulus of the $T^{2}$ respectively by $U$ and $T$. For $N=1$ it is easy to see that the theory exhibits a T-duality group

$$
\Gamma_{\text {het }}=\mathrm{SL}(2, \mathbb{Z}) \times \mathrm{SL}(2, \mathbb{Z}) \times \mathbb{Z}_{2} .
$$

One $\operatorname{SL}(2, \mathbb{Z})$ acts on the complex structure $U$ and must clearly leave the theory invariant. T-duality along one of the cycles exchanges $U$ with $T$ and therefore leads to the factor $\mathbb{Z}_{2}$. Combining both transformations it follows that the theory must also be invariant under the action of $\operatorname{SL}(2, \mathbb{Z})$ on $T$. In the dual Calabi-Yau geometry $U$ is identified with the Kähler modulus of an elliptic fiber while $T$ is the volume of the base of a $K 3$ fiber. We already argued that the $\mathrm{SL}(2, \mathbb{Z})$ action is realized by monodromies in the moduli space of elliptic fibrations and it is also known that another monodromy acts via $U \leftrightarrow T$ [43].

How does this situation change if we consider CHL orbifolds? The orbifold group $\mathbb{Z}_{N}$ acts as an order $N$ shift along one of the cycles of $T^{2}$ and the action of the duality group on 
the complex structure parameter of the torus has to be compatible with this action. But the subgroup of $\mathrm{SL}(2, \mathbb{Z})$ that preserves a subgroup $\mathbb{Z}_{N}$ of the torus together with a choice of generator is $\Gamma_{1}(N)$. On the other hand, we can still perform T-duality along the cycle that is not involved in the CHL quotient to exchange $U$ and $T$. We therefore conclude that the T-duality group of heterotic strings on a CHL orbifold $\left(K 3 \times T^{2}\right) / \mathbb{Z}_{N}$ is

$$
\Gamma_{\mathrm{CHL}}=\Gamma_{1}(N) \times \Gamma_{1}(N) \times \mathbb{Z}_{2} .
$$

From the perspective of the dual IIA compactification the action of this group should again be realized by the monodromies in the quantum Kähler moduli space of the CalabiYau. Together with our previous discussion in section 3.3 this implies that the Calabi-Yau has to be a genus one fibration with $N$-sections. For explicit examples that are dual to order 2 CHL compactifications we checked that $\mathbb{Z}_{2}$ is also contained in the corresponding monodromy group.

It is natural to ask under which conditions a weakly coupled CHL dual exists for a given $N$-section geometry. To shed some light on this question we construct all 2-section fibration over $\mathbb{P}^{1} \times \mathbb{P}^{1}$ with $h^{1,1}=3$ that can be realized as hypersurfaces in toric ambient spaces. This leads to two additional values of $\hat{b}$ for which the topological string amplitudes match with the calculation from the heterotic side. However, the list of gauge backgrounds that has been obtained by [50] is not exhaustive and we expect that a different construction should provide the missing heterotic duals. The two models found by [116] are contained in this list.

\subsection{Constructing Calabi-Yau duals of CHL orbifolds}

To keep the calculations tractable we focus on the case where the gauge symmetry on the heterotic side is maximally Higgsable. This means that only the four universal vector fields that arise from the Kaluza-Klein reduction of the metric and the $B$-field along the cycles of the 2-torus remain massless. The number of Kähler moduli for the dual Calabi-Yau manifolds is therefore $h^{1,1}=3$.

It has been argued on general grounds $[43,118]$ that weakly coupled heterotic strings can only be dual to Type IIA compactifications on Calabi-Yau manifolds that exhibit a $K 3$ fibration. The heterotic string arises in the IIA picture from a 5-brane that wraps the $K 3$ [119]. Further dualities relate the elliptic genus of this string to the topological string partition function which in turn encodes the couplings (5.1). Moreover, heterotic string theory on $K 3 \times T^{2}$ with $12+n$ and $12-n$ instantons embedded into the respective $E_{8}$ factors is dual to a compactification on an elliptic fibration over the Hirzebruch surfaces $\mathbb{F}_{n}$ [120]. Only for $n=0,1,2$ the gauge theory is maximally Higgsed at a generic point of the hypermultiplet moduli space.

All three geometries lead to the same string when a 5-brane wraps the restriction of the elliptic fibration to the fiber of the Hirzebruch surface. To search for Calabi-Yau duals of $\mathbb{Z}_{2}$ CHL orbifolds we are therefore constructing genus one fibrations over $\mathbb{F}_{1}=\mathbb{P}^{1} \times \mathbb{P}^{1}$ with 2 -sections. We can then study the strings from both of the $\mathbb{P}^{1}$ 's and compare with the predictions from [51]. 
Note that a genus one fibration with 2-sections can always be mapped into a fibration of degree 4 hypersurfaces in $\mathbb{P}_{112}$ using for example the techniques that have been reviewed in [66]. The systematic construction of elliptically and genus one fibered Calabi-Yau manifolds as hypersurfaces in toric ambient spaces is reviewed in section 7.1. Following that discussion we choose the fiber of the ambient space to be $\mathbb{P}_{112}$ and one can write down generic GLSM charge vectors as the rows of the matrix

$$
A=\left(\begin{array}{rrrrrrr}
1 & 1 & 2 & 0 & 0 & 0 & 0 \\
-q_{1} & -2 & -q_{1} & 1 & 1 & 0 & 0 \\
-q_{2} & -2 & -q_{2} & 0 & 0 & 1 & 1
\end{array}\right)
$$

where $q_{i}=q_{i}\left(\mathcal{S}_{7}-2 \mathcal{S}_{9}\right)$ in the notation introduced in and below (7.6). The kernel $B=$ $\operatorname{ker}(A)$ of this matrix is generated by the points of the polytope with integral points

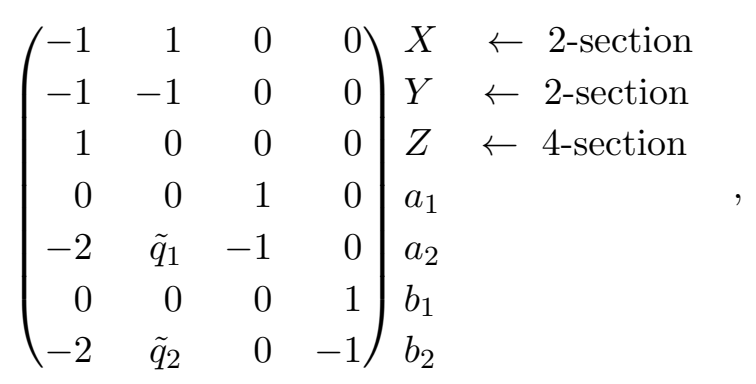

with $\tilde{q}_{i}=q_{i}-2$. We have already indicated the names for the homogeneous coordinates of the associated toric variety. The $\mathbb{P}^{1}$ in the base with coordinates $a_{1}, a_{2}$ will be denoted by $\mathbb{P}_{A}^{1}$ and the other will correspondingly be called $\mathbb{P}_{B}^{1}$. The Stanley-Reisner ideal is then always given by

$$
\mathcal{S R \mathcal { I }}=\left\langle a_{1} a_{2}, b_{1} b_{2}, X Y Z\right\rangle,
$$

and we denote the corresponding divisors by $D_{1}=\left[a_{1}\right]=\left[a_{2}\right], D_{2}=\left[b_{1}\right]=\left[b_{2}\right], E_{0}=[X]$. It is then easy to calculate

$$
E_{0}^{2} \cdot D_{1}=4-q_{2}, \quad E_{0}^{2} \cdot D_{2}=4-q_{1},
$$

and therefore

$$
\tilde{E}_{0}=E_{0}+\left(q_{1}-2\right) D_{1}+\left(q_{2}-2\right) D_{2} .
$$

We can expand the Kähler form as

$$
\omega=\tau \cdot \tilde{E}_{0}+\sum_{i=1}^{\infty}\left(t_{i}-\frac{\tilde{a}_{i}}{4}\right) \cdot D_{i},
$$

with

$$
\tilde{a}_{1}=\tilde{E}_{0}^{2} \cdot D_{2}=2 q_{1}-4, \quad \tilde{a}_{2}=\tilde{E}_{0}^{2} \cdot D_{1}=2 q_{2}-4
$$




\begin{tabular}{|rrrrrr|}
\hline$q_{1}$ & $q_{2}$ & $h^{1,1}$ & $h^{2,1}$ & $\hat{b}_{1}$ & $\hat{b}_{2}$ \\
\hline 4 & 4 & 4 & 148 & - & - \\
4 & 3 & 3 & 131 & 1 & - \\
4 & 2 & 3 & 115 & $\frac{8}{9}$ & $\frac{8}{9}$ \\
4 & 1 & 3 & 99 & $\frac{7}{9}$ & - \\
4 & 0 & 3 & 83 & $\frac{2}{3}$ & $\frac{2}{3}$ \\
\hline
\end{tabular}

\begin{tabular}{|rrrrrr|}
\hline$q_{1}$ & $q_{2}$ & $h^{1,1}$ & $h^{2,1}$ & $\hat{b}_{1}$ & $\hat{b}_{2}$ \\
\hline 3 & 3 & 3 & 123 & - & - \\
3 & 2 & 3 & 115 & - & $\frac{8}{9}$ \\
3 & 1 & 3 & 107 & - & - \\
2 & 2 & 3 & 115 & $\frac{8}{9}$ & $\frac{8}{9}$ \\
\hline
\end{tabular}

Table 1. Values for $q_{1}, q_{2}$ in (5.9) that lead to reflexive polytopes such that the corresponding toric variety is a $\mathbb{P}_{112}$ fibration over $\mathbb{P}^{1} \times \mathbb{P}^{1}$ and the generic Calabi-Yau hypersurface has $h^{1,1}=3$. When the string that arises from a 5 -brane wrapping the restriction of the genus one fibration to $\mathbb{P}_{A}$ or $\mathbb{P}_{B}$ matches with a $2 \mathrm{~A}$ CHL string, the corresponding value of $\hat{b}$ is also listed.

Only for a small set of values for $q_{1}, q_{2}$ the convex hull of the points is a reflexive polytope such that the corresponding toric variety is a $\mathbb{P}_{112}$ fibration over $\mathbb{P}^{1} \times \mathbb{P}^{1}$ and the generic Calabi-Yau hypersurface exhibits $h^{1,1}=3$. Moreover, two pairs of values can lead to polytopes that are identified under a lattice automorphism. We list a complete set of admissible values in table 1 that lead to inequivalent reflexive polytopes. The list also includes the unique special case where $h^{1,1}=4$ but one of the Kähler deformations is non-toric. This corresponds to the situation where the ramification locus of a toric 2 sections is trivial and it can be identified with the union of two independent sections. Note that a Calabi-Yau with $\left(h^{1,1}, h^{2,1}\right)=(3,131)$ has already been proposed in [39] as the dual geometry for a heterotic compactification on a particular $\left(K 3 \times T^{2}\right) / \mathbb{Z}_{2}$ orbifold.

Let us denote the corresponding geometries by $M_{q_{1}, q_{2}}$. Using the generic formula for the Euler characteristic from [85], the number of complex structure moduli can be expressed as

$$
h^{2,1}\left(q_{1}, q_{2}\right)=144+h^{1,1}\left(q_{1}, q_{2}\right)-16\left(q_{1}+q_{2}\right)+8 q_{1} q_{2} .
$$

Together with (5.4) and (5.5) this implies that if a 5-brane that wraps one of the $K 3$ fibers in $M_{q_{1}, q_{2}}$ can be identified with the string of a CHL orbifold of the previously discussed type, then the gauge background is necessarily such that

$$
\hat{b}=\frac{1}{144}\left[160-16\left(q_{1}+q_{2}\right)+8 q_{1} q_{2}\right] \text {. }
$$

Indeed, using the normal form [121] for reflexive polytopes one can easily check that the two geometries found by [116] respectively correspond to $\left(q_{1}, q_{2}\right)=(2,2)$ and $\left(q_{1}, q_{2}\right)=(4,0)$.

For any of the nine geometries we can consider the $K 3$ fiber with base $\mathbb{P}_{A}^{1}$ or $\mathbb{P}_{B}^{1}$ which we will sometimes denote by $K 3_{1}$ and $K 3_{2}$. This leads to eighteen base degree one partition functions and in all of the genuine genus one fibrations with $h^{1,1}=3$ it can either be written as

$$
Z_{1}^{(1)}(\tau, \lambda)=\frac{\Delta_{2}(\tau)}{\eta(2 \tau)^{24} \phi_{-2,1}(2 \tau, \lambda)} \cdot E_{2}^{(2)} \cdot\left[4(6 \hat{b}-5) \cdot\left(E_{2}^{(2)}\right)^{2}+2(2-3 \hat{b}) \cdot E_{4}\right]
$$

or

$$
Z_{1}^{(2)}(\tau, \lambda)=\frac{\Delta_{2}(\tau)^{\frac{3}{2}}}{\eta(2 \tau)^{24} \phi_{-2,1}(2 \tau, \lambda)} \cdot\left[\lambda_{1} \cdot\left(E_{2}^{(2)}\right)^{2}+\lambda_{2} \cdot E_{4}\right]
$$


As our notation already suggests, it is the first case that matches the predictions from a one-loop calculation in $2 A$ CHL orbifolds with a corresponding value for $\hat{b}$.

If we multiply $Z_{1}$ with $q^{\frac{\tilde{a}}{2 N}}$, see (3.32), the poles at $\tau \rightarrow i \infty$ are cancelled. Indeed we find that $Z_{1}^{(1)}$ is a valid ansatz for $K 3_{i}$ if

$$
\tilde{a}_{i} \equiv 0 \bmod 4
$$

and otherwise $Z_{1}^{(2)}$ matches the genus zero and genus one invariants for an appropriate choice of $\lambda_{1}, \lambda_{2}$. Expressions for $\tilde{a}_{i}, i=1,2$ in terms of $q_{1}, q_{2}$ have been provided in (5.14).

In the special case where $h^{1,1}=4$ the geometry is actually elliptically fibered with two independent sections that descend from one toric 2 -section. We then find that $K 3_{1}$ is equivalent to $K 3_{2}$ and the base degree one partition function reads

$$
Z_{1}(\tau, \lambda)=\frac{2}{3} \frac{\Delta_{2}(\tau)}{\eta(2 \tau)^{24} \phi_{-2,1}(2 \tau, \lambda)} \cdot E_{2}^{(2)} \cdot\left[\left(E_{2}^{(2)}\right)^{2}-4 E_{4}\right] .
$$

\subsection{Comparison with the heterotic one-loop computation}

The one-loop calculation of the couplings (5.1) has been carried out for many CHL orbifolds with standard-embedding of the gauge connection in [51]. However, all of the bundle dependent data that enters the result is contained in the new supersymmetric index. For many non-standard embeddings on $2 A$ orbifolds the index has been obtained in [50] and takes the form

$$
\mathcal{Z}_{\text {new }}=-4 \sum_{r, s=0}^{1} \Gamma_{2,2}^{(r, s)} f_{2 A}^{(r, s)}
$$

with $\Gamma_{2,2}^{(r, s)}$ a lattice sum that will not be relevant to us and

$$
\begin{aligned}
f_{2 A}^{(0,0)} & =\frac{1}{\eta(\tau)^{24}} \frac{E_{4} E_{6}}{2}, \\
f_{2 A}^{(0,1)} & =\frac{1}{\eta(\tau)^{24}} \frac{1}{4}\left[\left(E_{6}+2 E_{2}^{(2)}(\tau) E_{4}\right)\left(\hat{b}\left(E_{2}^{(2)}(\tau)\right)^{2}+\left(\frac{2}{3}-\hat{b}\right) E_{4}\right)\right], \\
f_{2 A}^{(1,0)} & =\frac{1}{\eta(\tau)^{24}} \frac{1}{4}\left[\left(E_{6}-E_{2}^{(2)}\left(\frac{\tau}{2}\right) E_{4}\right)\left(\frac{\hat{b}}{4}\left(E_{2}^{(2)}\left(\frac{\tau}{2}\right)\right)^{2}+\left(\frac{2}{3}-\hat{b}\right) E_{4}\right)\right], \\
f_{2 A}^{(1,1)} & =\frac{1}{\eta(\tau)^{24}} \frac{1}{4}\left[\left(E_{6}-E_{2}^{(2)}\left(\frac{\tau+1}{2}\right) E_{4}\right)\left(\frac{\hat{b}}{4}\left(E_{2}^{(2)}\left(\frac{\tau+1}{2}\right)\right)^{2}+\left(\frac{2}{3}-\hat{b}\right) E_{4}\right)\right],
\end{aligned}
$$

Recall that the choice of gauge background only enters via the combination $\hat{b}(5.4)$ of the numbers of vector- and hypermultiplets.

A direct evaluation of the one-loop amplitude leads to [51]

$$
\bar{F}_{g}^{\mathrm{hol}}=\frac{(-1)^{g-1}}{\pi^{2}} \sum_{s=0}^{N-1} \sum_{m}^{\prime} e^{-2 \pi i n_{2} s / N} c_{g-1}^{\left(n_{1}, s\right)}\left(\frac{n_{1} n_{2}}{2}, 0\right) \operatorname{Li}_{3-2 g}\left(e^{2 \pi i m \cdot y}\right)+\text { const. }
$$




\begin{tabular}{|c|ccccc|}
\hline$d \backslash g$ & 0 & 1 & 2 & 3 & 4 \\
\hline 0 & $512-288 \hat{b}$ & 0 & 0 & 0 & 0 \\
1 & $2376 \hat{b}+8128$ & $36 \hat{b}-32$ & 0 & 0 & 0 \\
2 & $151552-11520 \hat{b}$ & $576 \hat{b}-1024$ & 0 & 0 & 0 \\
3 & $45900 \hat{b}+1212576$ & $-4644 \hat{b}-16352$ & $48-54 \hat{b}$ & 0 & 0 \\
4 & $8671232-158976 \hat{b}$ & $24768 \hat{b}-306176$ & $1536-864 \hat{b}$ & 0 & 0 \\
5 & $493488 \hat{b}+47890048$ & $-105912 \hat{b}-2474048$ & $6840 \hat{b}+24640$ & $72 \hat{b}-64$ & 0 \\
6 & $240009216-1410048 \hat{b}$ & $389376 \hat{b}-18255872$ & $462848-39168 \hat{b}$ & $1152 \hat{b}-2048$ & 0 \\
7 & $3777570 \hat{b}+1055720304$ & $-1281132 \hat{b}-103120800$ & $174906 \hat{b}+3768496$ & $-8964 \hat{b}-32992$ & $80-90 \hat{b}$ \\
\hline
\end{tabular}

Table 2. Gopakumar-Vafa invariants of the $K 3$ at base degree 1 .

where $\sum^{\prime}$ is a sum over the points

$$
n_{1}, n_{2} \geq 0 \text {, but }\left(n_{1}, n_{2}\right) \neq(0,0), \text { and }\left(n_{1},-n_{2}\right) \text {, with } n_{2}>0 \text { and } n_{1} n_{2} \leq N \text {. }
$$

and $y=(T, U)$ contains the Kähler and complex structure of the torus. The coefficients $c_{g-1}^{(r, s)}(l, t)$ are defined via

$$
f^{(r, s)}(\tau) \mathcal{P}_{2 g+2}(\tau)=\sum_{l \in \frac{\mathbb{Z}}{N}, t=0}^{t=g} c_{g-1}^{(r, s)}(l, t) \tau_{2}^{-t} q^{l}
$$

where

$$
\mathcal{P}_{2 k}(\tau) \equiv \mathcal{P}_{2 k}\left(\hat{G}_{2}, \ldots, G_{2 k}\right), \quad G_{2 k}=2 \zeta(2 k) E_{2 k}, \quad \hat{E}_{2}(\tau)=E_{2}-\frac{3}{\pi \tau_{2}},
$$

and $\mathcal{P}_{2 k}\left(x_{1}, \ldots, x_{k}\right)$ is related to the Schur polynomial $\mathcal{S}_{k}\left(x_{1}, \ldots, x_{k}\right)$ by

$$
\mathcal{P}_{2 k}\left(x_{1}, \ldots, x_{k}\right)=-\mathcal{S}_{k}\left(x_{1}, \frac{1}{2} x_{2}, \ldots, \frac{1}{k} x_{k}\right) .
$$

The Schur polynomials are in turn defined by

$$
\exp \left(\sum_{k=1}^{\infty} x_{k} z^{k}\right)=\sum_{k=0}^{\infty} \mathcal{S}_{k}\left(x_{1}, \ldots, x_{k}\right) z^{k} .
$$

The indices $(r, s)$ label the twisted sectors and are considered modulo 2. From this we can extract all Gopakumar-Vafa invariants that have base degree zero with respect to the $K 3$ fibration of the dual Calabi-Yau up to arbitrary genus. The invariants of base degree 1 with respect to the genus one fibration of the $K 3$ are listed in the table 2 . We find perfect agreement with the predictions from (5.17).

The higher base degrees match with the results from the genus zero and genus one partition functions and can be used to fix the coefficients in the modular ansatz. Evaluation of (5.23) provide the prediction of Gopakumar-Vafa invariants listed in table 3. 


\begin{tabular}{|c|cccc|}
\hline$d \backslash g$ & 0 & 1 & 2 & 3 \\
\hline 0 & $288 \hat{b}-32$ & 4 & 0 & 0 \\
1 & $151552-11520 \hat{b}$ & $576 \hat{b}-1024$ & 0 & 0 \\
2 & $158976 \hat{b}+8387328$ & $-24768 \hat{b}-262464$ & $864 \hat{b}-128$ & 8 \\
3 & $240009216-1410048 \hat{b}$ & $389376 \hat{b}-18255872$ & $462848-39168 \hat{b}$ & $1152 \hat{b}-2048$ \\
4 & $9596160 \hat{b}+4294949632$ & $-3870144 \hat{b}-526389312$ & $674208 \hat{b}+27261728$ & $-54720 \hat{b}-524144$ \\
5 & $57704112128-54369792 \hat{b}$ & $29089152 \hat{b}-10135607296$ & $865636352-7319808 \hat{b}$ & $1016064 \hat{b}-39292928$ \\
6 & $268600320 \hat{b}+620790389760$ & $-179967744 \hat{b}-143003775232$ & $59660928 \hat{b}+17075001600$ & $-11892672 \hat{b}-1217397536$ \\
7 & $5647463645184-1191462912 \hat{b}$ & $962118144 \hat{b}-1626724835328$ & $253765050368-397518336 \hat{b}$ & $104037120 \hat{b}-25542406144$ \\
\hline
\end{tabular}

Table 3. Gopakumar-Vafa invariants of the $K 3$ at base degree 2 .

The Gopakumar-Vafa invariants in table 3 can be used to fix the numerator in our Ansatz for the base degree 2 partition function

$$
Z_{2}=\left(\frac{\Delta_{4}}{\eta^{24}(2 \tau)}\right)^{2} \frac{\phi_{2}(\tau, \lambda)}{\phi_{-2,1}(2 \tau, \lambda) \phi_{-2,1}(4 \tau, \lambda)},
$$

and we find that

$$
\begin{aligned}
\phi_{2}= & \frac{1}{82944}\left[384 A^{3}\left(2(6 \hat{b}-5) g^{3}+(2-3 \hat{b}) g h\right)^{2}+8 A^{2} B g\left(24(62 \hat{b}-55) g^{6}+10(95-108 \hat{b}) g^{4} h\right.\right. \\
& \left.+(261 \hat{b}-220) g^{2} h^{2}+7(2-3 \hat{b}) h^{3}\right)+A B^{2}\left(-48\left(4320 \hat{b}^{2}-6644 \hat{b}+2505\right) g^{8}+8(36 \hat{b}(504 \hat{b}\right. \\
& -703)+8321) g^{6} h+(36(1159-936 \hat{b}) \hat{b}-11231) g^{4} h^{2}+2(12 \hat{b}(108 \hat{b}-109)+179) g^{2} h^{3} \\
& \left.+3(11-12 \hat{b}) h^{4}\right)+4 B^{3} g\left(11 g^{2}-3 h\right)\left(12(8 \hat{b}-7)(36 \hat{b}-25) g^{6}+(216(11-8 \hat{b}) \hat{b}-763) g^{4} h\right. \\
& \left.\left.+(27 \hat{b}(8 \hat{b}-9)+50) g^{2} h^{2}+(5-3 \hat{b}) h^{3}\right)\right]
\end{aligned}
$$

where we introduced

$$
A=\phi_{0,1}(2 \tau, \lambda), \quad B=\phi_{-2,1}(2 \tau, \lambda), \quad g=E_{2}^{(2)}(\tau), \quad h=E_{4}(\tau) .
$$

We can thus check the duality between the proposed pairs of Type IIA compactifications on genus one fibrations with 2 -sections and heterotic compactifications on $(K 3 \times$ $\left.T^{2}\right) / \mathbb{Z}_{2}$, at least perturbatively, to arbitrary orders. The contributions to the topological string partition function that arise from curves that also wrap the base of the $K 3$-fibration correspond to a highly non-trivial prediction of the non-perturbative corrections on the heterotic side.

\section{Decoupling gravity on genus one fibrations over (-1)-curves}

During the last few years there has been a considerable amount of interest in the study of topological strings on non-compact Calabi-Yau manifolds that are elliptically fibered over 
curves of negative self-intersection $[6,15,18,19,122-124]$. The reason is the relation to elliptic genera of strings in so-called minimal six-dimensional $(1,0)$-superconformal field theories that serve as building blocks in a classification of all such theories [57, 58, 125]. Those strings arise in F-theory from D3-branes that wrap the curve in the base of the fibration. A natural question is, what happens if we consider a genus one fibration with multi-sections over a curve of negative self-intersection?

Arguably the simplest but most important building block arises from a non-compact fibration without reducible fibers over a curve of self-intersection -1 . The corresponding topological string partition function at base degree $d_{B}$ encodes the elliptic genus of an ensemble of $d_{B}$ E-strings and the SCFT can be used to glue theories in the atomic classification. In this section we will initiate the study of genus one-fibrations over $(-n)$-curves by discussing the analogues to the E-string geometry with $N$-sections for $N=2,3,4$.

\subsection{The refined topological string partition function}

The enumerative invariants of an elliptic or genus one fibered Calabi-Yau encode the BPSspectrum of particles that arise from the strings wrapped along the circle after compactifying from six to five dimensions. More precisely, the Gopakumar-Vafa invariants (4.34) correspond to a weighted sum of multiplicities of BPS states. The multiplicities themselves are not invariant and the number of BPS states with a particular mass and spin does, in general, jump across lines of marginal stability in the complex structure moduli space. However, non-compact Calabi-Yau manifolds are rigid and the topological string partition function can be refined such that it encodes the actual number of BPS states for a given set of quantum numbers.

Physically, the situation is as follows. M-theory compactified on a Calabi-Yau threefold $M$ leads to a 5 d supergravity with eight supercharges. The five-dimensional little group is $\mathrm{SO}(4)=\mathrm{SU}(2)_{L} \times \mathrm{SU}(2)_{R}$ and when gravity is decoupled an additional $\mathrm{SU}(2)_{I}$ R-symmetry emerges. The decoupling limit corresponds to a decompactification of the Calabi-Yau. Let us denote with $J_{*}$ the Cartan generator of $\mathrm{SU}(2)_{*}$. Then $J_{I}$ enables a twisting of $J_{R}$ such that the degeneracies of the BPS states $N_{j_{L}, j_{R}^{\prime}}^{\kappa}$ are protected [126]. In the following, $j_{R}$ will denote the spin with respect to the twisted $\mathrm{SU}(2)_{R}$ with Cartan generator $J_{R}+J_{I}$. The refined BPS states with multiplicities $N_{j_{L}, j_{R}}^{\kappa}$ are then labeled by the mass, which is specified by $\kappa \in H_{2}(X, \mathbb{Z})$, and the twisted spin representation $\left[j_{L}, j_{R}\right]$. They are encoded in the index

$$
\mathcal{Z}\left(\epsilon_{L}, \epsilon_{R}, \boldsymbol{t}\right)=\operatorname{Tr}_{\mathcal{B P S}}(-1)^{2\left(J_{L}+J_{R}\right)} e^{-2 \epsilon_{-} J_{L}} e^{-2 \epsilon_{+}\left(J_{R}+J_{I}\right)} e^{\beta H}
$$

which is called the refined topological string partition function.

From the perspective of the effective theory, $\mathcal{Z}$ can be obtained from a refinement of the Schwinger-Loop calculation in [127], which was performed by [126]. The result is

$$
\log \left(\mathcal{Z}\left(\epsilon_{1}, \epsilon_{2}, \boldsymbol{t}\right)\right)=\sum_{\kappa \in H_{2}(X, \mathbb{Z})} \sum_{m>0}(-1)^{g_{L}+g_{R}+1} \frac{n_{g_{L}, g_{R}}^{\kappa}}{m} \frac{\left(\sin \frac{m \epsilon_{-}}{2}\right)^{2 g_{L}}\left(\sin \frac{m \epsilon_{+}}{2}\right)^{2 g_{R}}}{\sin \frac{m \epsilon_{1}}{2} \sin \frac{m \epsilon_{2}}{2}} \boldsymbol{Q}^{m \kappa}
$$


where $n_{g_{L}, g_{R}}^{\kappa}$ are called refined Gopakumar-Vafa invariants and we introduced $\epsilon_{i}, i=1,2$ with $\epsilon_{ \pm}=\frac{1}{2}\left(\epsilon_{1} \pm \epsilon_{2}\right)$. They are related to the refined BPS invariants $N_{j_{L}, j_{R}}^{\kappa}$ via

$$
\sum_{j_{L}, j_{R} \in \frac{\mathbb{N}}{2}} N_{j_{L}, j_{R}}^{\kappa} \chi_{j_{L}}\left(x_{L}\right) \chi_{j_{R}}\left(x_{R}\right)=\sum_{g_{L}, g_{R} \in \mathbb{N}} n_{g_{L}, g_{R}}^{\kappa}\left(x_{L}^{\frac{1}{2}}-x_{L}^{-\frac{1}{2}}\right)^{2 g_{L}}\left(x_{R}^{\frac{1}{2}}-x_{R}^{-\frac{1}{2}}\right)^{2 g_{R}}
$$

where

$$
\chi_{j_{*}}\left(x_{*}\right)=\frac{x_{*}^{2 j_{*}+1}-x_{*}^{-2 j_{*}-1}}{x_{*}-x_{*}^{-1}},
$$

and $x_{*}$ is a formal variable. One can also define refined free energies $\mathcal{F}^{(n, g)}(\boldsymbol{t})$ via

$$
\log \left(\mathcal{Z}\left(\epsilon_{1}, \epsilon_{2}, \boldsymbol{t}\right)\right)=\sum_{n, g=0}^{\infty}\left(\epsilon_{1}+\epsilon_{2}\right)^{2 n}\left(\epsilon_{1} \epsilon_{2}\right)^{g-1} \mathcal{F}^{(n, g)}(\boldsymbol{t})
$$

In the limit $\epsilon_{+} \rightarrow 0,(6.2)$ reproduces the instantons part of the unrefined topological string partition function in (4.34) and the unrefined Gopakumar-Vafa invariants are

$$
n_{g}^{\beta}=(-1)^{g} \sum_{g_{R}} n_{g, g_{R}}^{\kappa} .
$$

The refined topological string partition function can also be expanded in terms of base parameters $Q^{\beta}, \beta \in H_{2}(B)$

$$
\mathcal{Z}\left(\epsilon_{1}, \epsilon_{2}, \boldsymbol{t}, \tau, \boldsymbol{m}\right)=\mathcal{Z}_{0}\left(1+\sum_{\beta \in H_{2}(B)} \mathcal{Z}_{\beta}\left(\epsilon_{1}, \epsilon_{2}, \tau, \boldsymbol{m}\right) Q^{\beta}\right) .
$$

The coefficient $\mathcal{Z}_{\beta}\left(\epsilon_{1}, \epsilon_{2}, \tau, \boldsymbol{m}\right)$ corresponds to the elliptic genus of a string that wraps the class $\beta$ in an F-theory compactification on $M[6,14,15]$. Moreover, after compactifying on an additional circle the theory is dual to M-theory on $M$ and the wrapped strings lead to the BPS particles that are counted by the refined invariants.

\subsection{Genus one fibrations and E-strings with Wilson lines}

We construct the non-compact fibration as local geometries in compact genus one fibered Calabi-Yau threefolds over the base $B=\mathbb{F}_{1}$. One can then show that the ramification divisor of the $N$-section intersects the $(-1)$-curve and therefore we expect the local geometry to be genus one fibered as well. We only consider maximally Higgsed geometries with only one linearly independent $N$-section and no fibral divisors. It seems to be the case that the results are then independent of the global structure of the fibration. We will therefore only consider one Calabi-Yau for each value $N=2,3,4$.

More precisely, we will consider three genus one fibrations with $N$-sections

$$
M_{N}=\left\{\begin{array}{l}
\left(F_{4} \rightarrow \mathbb{F}_{1}\right)\left[\mathbb{Z}_{2}\right]_{3}^{-224} \text { for } N=2 \\
\left(F_{1} \rightarrow \mathbb{F}_{1}\right)\left[\mathbb{Z}_{3}\right]_{3}^{-144} \text { for } N=3 \\
\left(\mathbb{P}^{3} \rightarrow \mathbb{F}_{1}\right)\left[\mathbb{Z}_{4}\right]_{3}^{-104} \text { for } N=4
\end{array} .\right.
$$


We also consider three elliptic fibrations with "pseudo- $N$-section". By this we mean elliptically fibered Calabi-Yau 3-folds that are realized as hypersurfaces or complete intersections in toric ambient spaces but exhibit non-toric divisors such that from the toric perspective multiple sections or merged into an $N$-section with trivial monodromy. The corresponding geometries are

$$
M_{N}^{\prime}=\left\{\begin{array}{l}
\left(F_{4} \rightarrow \mathbb{F}_{1}\right)\left[\mathbb{Z}_{2}\right]_{4(1)}^{-288} \text { for } N=2, \\
\left(F_{1} \rightarrow \mathbb{F}_{1}\right)\left[\mathbb{Z}_{3}\right]_{5(2)}^{-192} \text { for } N=3, \\
\left(\mathbb{P}^{3} \rightarrow \mathbb{F}_{1}\right)\left[\mathbb{Z}_{4}\right]_{6(3)}^{-128} \text { for } N=4 .
\end{array}\right.
$$

All of the geometries are hypersurfaces in toric ambient spaces except for $M_{4}$ and $M_{4}^{\prime}$ which are complete intersections. We will not discuss the geometries in detail but provide the toric data in D. In each case we want to consider the local limit of the base $\hat{B}=\mathcal{O}(-1) \rightarrow \mathbb{P}^{1}[15]$. This is achieved when $\operatorname{Vol}\left(C_{F}\right) \rightarrow \infty$, where $C_{F}$ is the class of the fiber in $\mathbb{F}_{1}$. We denote the $(-1)$-curve in the base of $\mathbb{F}_{1}$ by $C_{B}$ and let $\beta=b \cdot C_{B} \in H_{2}(\hat{B}, \mathbb{Z})$.

From our discussion of the modular ansatz for genus one fibrations in section 4.5 , we expect that one can obtain the ansatz for genus one fibrations over $(-1)$-curves from the E-string ansatz $[17,18]$ by substituting $\tau \rightarrow N \tau$. The result is

$$
\mathcal{Z}_{\beta}^{(N)}\left(\tau, \epsilon_{+}, \epsilon_{-}\right)=\frac{1}{\eta(N \tau)^{12 c_{1}(B) \cdot \beta}} \frac{\varphi_{\beta}\left(\tau, \epsilon_{+}, \epsilon_{-}\right)}{\prod_{\ell=1}^{b_{2}(B)} \prod_{s=1}^{\beta_{\ell}}\left[\phi_{-1, \frac{1}{2}}\left(N \tau, s \epsilon_{1}\right) \phi_{-1, \frac{1}{2}}\left(N \tau, s \epsilon_{2}\right)\right]} .
$$

where we have set the mass parameters of the E-string to zero. The numerator is now an element

$$
\varphi_{\beta} \in M_{*}(N)\left[\phi_{-2,1}(N \tau, \bullet), \phi_{0,1}(N \tau, \bullet)\right] \cdot \Delta_{2 N}^{1-\frac{r_{\beta}}{N}} \bmod 1,
$$

where $\bullet \in\left\{\epsilon_{+}, \epsilon_{-}\right\}$, such that the overall indices of $\mathcal{Z}_{\beta}^{(N)}$ with respect to $\epsilon_{-}, \epsilon_{+}$are

$$
r_{\beta}^{-}=\frac{1}{2} \beta \cdot\left(\beta-c_{1}(B)\right), \quad r_{\beta}^{+}=\frac{1}{2} \beta \cdot\left(\beta+c_{1}(B)\right)-2(\mathbf{1} \cdot \beta) .
$$

Recall that the exponent of $\Delta_{2 N}$ was defined via the congruence relation (4.92). The indices of the numerator with respect to $\epsilon_{+}$and $\epsilon_{-}(6.12)$ are

$$
r_{\beta}^{+}=\frac{b}{3}\left(b^{2}+3 b-4\right), \quad r_{\beta}^{-}=\frac{b}{3}\left(b^{2}-1\right) .
$$

Let us denote the numerators in $\mathcal{Z}_{b \cdot C_{B}}\left(\tau, \epsilon_{+}, \epsilon_{-}\right)$for $M_{N}$ and $M_{N}^{\prime}$ by $\phi_{b}^{(N)}$ and $\phi_{b}^{\left(N^{\prime}\right)}$. We then find that

$$
\begin{aligned}
& \phi_{1}^{(1)}(\tau)=-\frac{E_{4}(\tau)}{\eta(\tau)^{12}}, \\
& \phi_{1}^{(2)}(\tau)=-16 \frac{\Delta_{4}(\tau)}{\eta(2 \tau)^{12}}, \quad \phi_{1}^{\left(2^{\prime}\right)}(\tau)=-2 \frac{\sqrt{\Delta_{4}(\tau)} E_{2}^{(2)}(\tau)}{\eta(2 \tau)^{12}}, \\
& \phi_{1}^{(3)}(\tau)=-9 \frac{\Delta_{6}(\tau)^{\frac{2}{3}}}{\eta(3 \tau)^{12}}, \quad \phi_{1}^{\left(3^{\prime}\right)}(\tau)=-\frac{3}{216} \frac{E_{4}(\tau)-E_{2}^{(3)}(\tau)^{2}}{\eta(3 \tau)^{12}}, \\
& \phi_{1}^{(4)}(\tau)=-\frac{1}{2} \frac{\Delta_{8}(\tau)^{\frac{1}{4}}\left(E_{2}^{(2)}(\tau)-E_{2}^{(4)}(\tau)\right)}{\eta(4 \tau)^{12}}, \quad \phi_{1}^{\left(4^{\prime}\right)}(\tau)=-4 \frac{\sqrt{\Delta_{8}(\tau)}}{\eta(4 \tau)^{12}}
\end{aligned}
$$


where $N=1$ corresponds to the ordinary E-string. It turns out that the elliptic genus $\mathcal{Z}_{1}^{(2)}$ already appeared in [59] where it was obtained from the E-string on a circle with a non-zero Wilson loop for the affine $E_{8}$-flavor symmetry turned on. More generally, up to overall factors of $q$ we obtain the expression

$$
\phi_{1}^{(N)}(\tau)=-\frac{2}{\eta(N \tau)^{12}} \sum_{i=2}^{4} \prod_{j=1}^{8} \theta_{i}\left(N \tau, v_{j}^{(N)} \cdot \tau\right),
$$

with Wilson loop parameters $\vec{v}^{(N)}$ given by

$$
\begin{array}{ll}
\vec{v}^{(1)}=(0,0,0,0,0,0,0,0), \\
\vec{v}^{(2)}=(0,0,0,0,0,0,0,2), & \vec{v}^{\left(2^{\prime}\right)}=(0,0,0,0,0,0,1,1), \\
\vec{v}^{(3)}=(0,0,0,1,1,1,1,2), & \vec{v}^{\left(3^{\prime}\right)}=(0,0,0,0,0,1,1,2), \\
\vec{v}^{(4)}=(0,0,0,1,1,2,2,2), & \vec{v}^{\left(4^{\prime}\right)}=(0,0,0,0,1,1,1,3) .
\end{array}
$$

The definition of the Jacobi theta functions $\theta_{i}(\tau, z), i=1, \ldots, 4$ was given in (4.16). Estrings with wilson lines have also been discussed in [128].

Moreover, we provide the numerator $\phi_{b}^{(N)}$ of $M_{2}$ for base degree $b=2$ :

$$
\begin{aligned}
\phi_{2}^{(2)}= & \frac{\Delta_{4}^{2}}{\eta^{24}(2 \tau)}\left(-\frac{497 \mathcal{A}_{+, 2}^{4} \mathcal{A}_{-, 2}^{2} E_{2,2}^{6}}{373248}+\frac{29 \mathcal{A}_{+, 2}^{4} \mathcal{A}_{-, 2}^{2} E_{2,2}^{4} E_{4}}{124416}+\frac{13 \mathcal{A}_{+, 2}^{4} \mathcal{A}_{-, 2}^{2} E_{2,2}^{2} E_{4}^{2}}{41472}-\frac{\mathcal{A}_{+, 2}^{4} \mathcal{A}_{-, 2}^{2} E_{4}^{3}}{13824}\right. \\
& +\frac{127 \mathcal{A}_{+, 2}^{4} \mathcal{A}_{-, 2} \mathcal{B}_{-, 2} E_{2,2}^{5}}{93312}-\frac{35 \mathcal{A}_{+, 2}^{4} \mathcal{A}_{-, 2} \mathcal{B}_{-, 2} E_{2,2}^{3} E_{4}}{31104}+\frac{\mathcal{A}_{+, 2}^{4} \mathcal{A}_{-, 2} \mathcal{B}_{-, 2} E_{2,2} E_{4}^{2}}{5184}-\frac{\mathcal{A}_{+, 2}^{4} \mathcal{B}_{-, 2}^{2} E_{2,2}^{4}}{2916} \\
& +\frac{5 \mathcal{A}_{+, 2}^{4} \mathcal{B}_{-, 2}^{2} E_{2,2}^{2} E_{4}}{13824}-\frac{\mathcal{A}_{+, 2}^{4} \mathcal{B}_{-, 2}^{2} E_{4}^{2}}{13824}+\frac{533 \mathcal{A}_{+, 2}^{3} \mathcal{A}_{-, 2}^{2} \mathcal{B}_{+, 2} E_{2,2}^{5}}{93312}-\frac{23 \mathcal{A}_{+, 2}^{3} \mathcal{A}_{-, 2}^{2} \mathcal{B}_{+, 2} E_{2,2}^{3} E_{4}}{10368}+\frac{\mathcal{A}_{-, 2}^{2} \mathcal{B}_{+, 2}^{4} E_{4}}{10368} \\
& +\frac{\mathcal{A}_{+, 2}^{3} \mathcal{A}_{-, 2}^{2} \mathcal{B}_{+, 2} E_{2,2} E_{4}^{2}}{5184}-\frac{257 \mathcal{A}_{+, 2}^{3} \mathcal{A}_{-, 2} \mathcal{B}_{+, 2} \mathcal{B}_{-, 2} E_{2,2}^{4}}{46656}+\frac{55 \mathcal{A}_{+, 2}^{3} \mathcal{A}_{-, 2} \mathcal{B}_{+, 2} \mathcal{B}_{-, 2} E_{2,2}^{2} E_{4}}{20736}+\frac{\mathcal{A}_{-, 2} \mathcal{B}_{+, 2}^{4} \mathcal{B}_{-, 2} E_{2,2}}{5832} \\
& -\frac{7 \mathcal{A}_{+, 2}^{3} \mathcal{A}_{-, 2} \mathcal{B}_{+, 2} \mathcal{B}_{-, 2} E_{4}^{2}}{20736}+\frac{29 \mathcal{A}_{+, 2}^{3} \mathcal{B}_{+, 2} \mathcal{B}_{-, 2}^{2} E_{2,2}^{3}}{23328}-\frac{\mathcal{A}_{+, 2}^{3} \mathcal{B}_{+, 2} \mathcal{B}_{-, 2}^{2} E_{2,2} E_{4}}{2592}-\frac{193 \mathcal{A}_{+, 2}^{2} \mathcal{A}_{-, 2}^{2} \mathcal{B}_{+, 2}^{2} E_{2,2}^{4}}{31104} \\
& +\frac{25 \mathcal{A}_{+, 2}^{2} \mathcal{A}_{-, 2}^{2} \mathcal{B}_{+, 2}^{2} E_{2,2}^{2} E_{4}}{13824}-\frac{\mathcal{A}_{+, 2}^{2} \mathcal{A}_{-, 2}^{2} \mathcal{B}_{+, 2}^{2} E_{4}^{2}}{13824}+\frac{11 \mathcal{A}_{+, 2}^{2} \mathcal{A}_{-, 2} \mathcal{B}_{+, 2}^{2} \mathcal{B}_{-, 2} E_{2,2}^{3}}{1944}-\frac{1}{648} \mathcal{A}_{+, 2}^{2} \mathcal{A}_{-, 2} \mathcal{B}_{+, 2}^{2} \mathcal{B}_{-, 2} E_{2,2} E_{4} \\
& -\frac{7 \mathcal{A}_{+, 2}^{2} \mathcal{B}_{+, 2}^{2} \mathcal{B}_{-, 2}^{2} E_{2,2}^{2}}{7776}+\frac{\mathcal{A}_{+, 2}^{2} \mathcal{B}_{+, 2}^{2} \mathcal{B}_{-, 2}^{2} E_{4}}{10368}+\frac{59 \mathcal{A}_{+, 2} \mathcal{A}_{-, 2}^{2} \mathcal{B}_{+, 2}^{3} E_{2,2}^{3}}{23328}-\frac{5 \mathcal{A}_{+, 2} \mathcal{A}_{-, 2}^{2} \mathcal{B}_{+, 2}^{3} E_{2,2} E_{4}}{7776}+\frac{\mathcal{B}_{+, 2}^{4} \mathcal{B}_{-, 2}^{2}}{5832} \\
& \left.-\frac{23 \mathcal{A}_{+, 2} \mathcal{A}_{-, 2} \mathcal{B}_{+, 2}^{3} \mathcal{B}_{-, 2} E_{2,2}^{2}}{11664}+\frac{7 \mathcal{A}_{+, 2} \mathcal{A}_{-, 2} \mathcal{B}_{+, 2}^{3} \mathcal{B}_{-, 2} E_{4}}{15552}-\frac{\mathcal{A}_{+, 2} \mathcal{B}_{+, 2}^{3} \mathcal{B}_{-, 2}^{2} E_{2,2}}{5832}-\frac{\mathcal{A}_{-, 2}^{2} \mathcal{B}_{+, 2}^{4} E_{2,2}^{2}}{2916}\right)
\end{aligned}
$$

Here we introduced the compact notation $\mathcal{A}_{ \pm, N} \equiv \phi_{-2,1}\left(N \tau, \epsilon^{ \pm}\right), \mathcal{B}_{ \pm, N} \equiv \phi_{0,1}\left(N \tau, \epsilon^{ \pm}\right)$, and $E_{2}^{(N)} \equiv E_{2, N}$. Analogous expressions for the case $M_{3}$ and $M_{4}$ can be found in appendix E. Expressions $\phi_{b}^{(N)}$ for $M_{N}^{\prime}$ are quite similar to the $M_{N}$ cases and hence we omit them. Instead, we provide some refined BPS invariants in appendix $\mathrm{F}$ up to $b=2$. We provide a notebook online [129] that contains higher degree invariants up to $b=3$ for all cases that we consider.

Note that the E-string has a dual interpretation in heterotic $E_{8} \times E_{8}$ theory. From the Horava-Witten perspective it arises from M2-branes that are stretched between an M5 and M9 brane on the interval that is used to compactify M-theory. If we consider a generic elliptic fibration over the Hirzebruch surface $\mathbb{F}_{1}$, the E-string arises from D3-branes wrapping the $(-1)$-curve while the corresponding heterotic string arises from D3-branes 
that wrap the $\mathbb{P}^{1}$ fiber of the base. In the previous section we have matched the invariants from the restriction of the genus-one fibration to the fiber $\mathbb{P}^{1}$ for 2 -section geometries with the one-loop calculation in a heterotic compactification on $\left(K 3 \times T^{2}\right) / \mathbb{Z}_{N}$. It is therefore natural to expect an interpretation of the flavour group for the strings from the $(-1)$ curve in terms of small instantons in the corresponding CHL-model. We leave a detailed investigation of this question to future work.

\section{Examples}

In this section we will illustrate the general discussion of the previous sections with several examples of genus one fibered Calabi-Yau threefolds. One set of geometries is related via a chain of birational transformations that do not change the base of the fibration. In the effective action of the corresponding F-theory compactification this is manifested as Higgsing and un-Higgsing the gauge group. We will therefore use the name Higgs chain for a set of genus one fibered Calabi-Yau manifolds that are related via extremal transitions which do not involve blow ups or blow downs of the base. Moreover, when we refer to the gauge group of a geometry this is to be understood as the gauge group of the corresponding F-theory effective action.

All of our examples are obtained using the fiber based construction that we review in the following section 7.1. We use the notation

$$
\left(F_{i} \rightarrow B\right)[G]_{h^{1,1}}^{\chi},
$$

for a genus one fibered Calabi-Yau with base $B$ such that the generic fiber is constructed as a hypersurface in $\mathbb{P}_{F_{i}}$ The gauge group of the corresponding F-theory vacuum is $G$ and the hodge numbers are determined by $h^{1,1}$ and the Euler characteristic $\chi$. If some of the divisors are not torically realized we indicate this by writing e.g. $h^{1,1}=3(1)$ if one out of three generators is non-toric.

In general this data does not determine the geometry uniquely. The reason is that for a given fiber polytope the geometry is determined by fixing two line bundles on the base. Since the number of divisors and the gauge group are the same for all sufficiently generic choices of the bundles there is only one constraint imposed by the Euler characteristic. However, in all the cases that we discuss in this section the bundles are non-generic and there is no such ambiguity.

This being said, we will discuss the Higgs chain that includes geometries with the data listed in (7.2):

$$
\left(F_{10} \rightarrow \mathbb{P}^{2}\right)[\mathrm{SU}(2)]_{3}^{-216} \rightarrow\left(F_{6} \rightarrow \mathbb{P}^{2}\right)[\mathrm{U}(1)]_{3}^{-216} \rightarrow\left(F_{4} \rightarrow \mathbb{P}^{2}\right)\left[\mathbb{Z}_{2}\right]_{2}^{-252}
$$

But first we are going to review the general strategy to construct elliptic and genus one fibered Calabi-Yau manifolds with the tools of toric geometry.

\subsection{Eliptically fibered Calabi-Yau as toric hypersurfaces}

Still the most abundant source of Calabi-Yau threefolds are hypersurfaces and complete intersections in toric ambient spaces. The Batyrev construction associates a mirror pair of $d$ dimensional Calabi-Yau hypersurfaces to every $d+1$-dimensional reflexive polyotope [130]. 

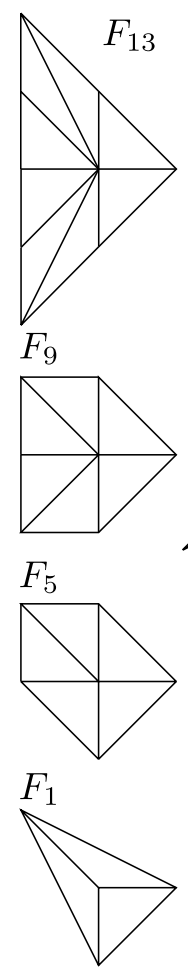

$F_{14}$

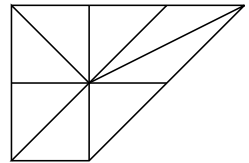

$F_{15}$

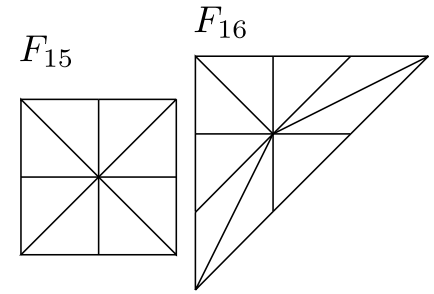

$F_{10}$

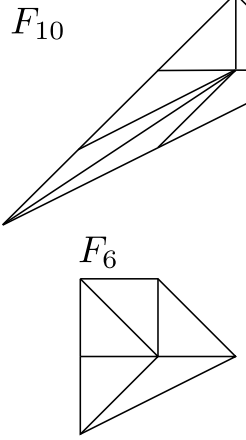

$F_{2}$

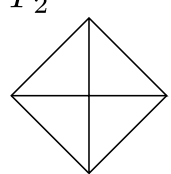

$F_{12}$

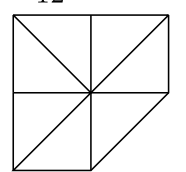

$F_{8}$
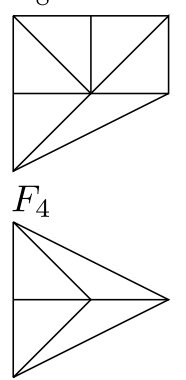

Figure 4. The 16 two-dimensional reflexive polytopes. The image is taken from [85].

As a slight abuse of terminology we will call such varieties toric Calabi-Yau hypersurfaces. Four dimensional reflexive polytopes have been fully classified and the complete Kreuzer-Skarke list with 473,800,776 entries is available online [131]. On the other hand, complete intersection Calabi-Yau $d$-folds of codimension $m$ are related to nef-partitions of $d+m$-dimensional reflexive polytopes [61]. For recent progress on the classification of five-dimensional reflexive polytopes see [132].

A given Calabi-Yau $X$ is said to be genus one fibered over a base $B$ if there exists a surjective map $\pi: X \rightarrow B$ such that the generic fiber over $B$ is a torus. If the projection $\pi$ admits a section then the fibration is called elliptic. It is well known that the majority of toric Calabi-Yau hypersurfaces is genus one fibered in at least one and often multiple ways. For a recent discussion and pointers to the literature see e.g. [133]. To obtain explicit examples of genus one fibered Calabi-Yau hypersurfaces there are three general strategies:

1. It is possible to manually engineer polytopes that correspond to toric fibrations such that the generic hypersurface cuts out a genus one curve from the generic fiber.

2. For a given reflexive polytope one can systematically search for the toric fibrations of the corresponding toric variety. This has been used by [134] and [132] to scan for fibrations in the complete Kreuzer-Skarke list.

3. One can first construct an elliptic curve as a hypersurface in a toric variety. The coefficients of the hypersurface equation can then be lifted to sections of line bundles 
a), $M$

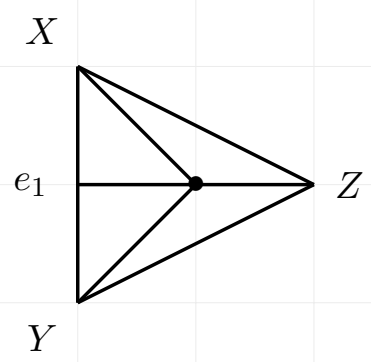

b), $N$

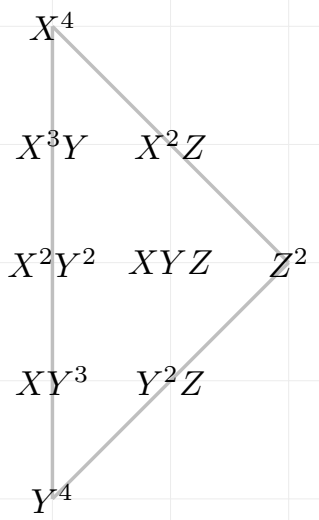

Figure 5. The dual pair of polytopes $F_{4}$ and $F_{13}$ is shown in a) and b). We also indicate the toric fan obtained from a complete star triangulation of $F_{4}$ and labelled the homogeneous coordinates. In the monomials that correspond to points of $F_{13}$ we have set $e_{1}=1$. The dependence can be easily restored.

over some base that we will also assume to be toric. For every pair of fiber and base this leads to a finite number of "reasonable" choices. This is what we refer to as the "fiber based approach".

The fiber based construction has the advantage that it is quite general and fibrations with very specific properties can be engineered. A comphrehensive study of the properties of the fibers that can be obtained from the 16 two-dimensional reflexive polytopes that are shown in figure 4 has been performed in [85] and we will frequently refer to these results. Furthermore, a reflexive polytope that corresponds to the total space can often be recovered from the choice of fiber, base and bundles.

We will now review the general construction at the hand of a particular example. Note that although we only work with hypersurfaces the discussion can be easily generalized to complete intersections. Figure 5 shows the dual pair of reflexive polytopes $F_{4}$ and $F_{13}$ that are subsets of the respective lattices $M$ and $N$. We construct the generic fiber as a hypersurface in the toric variety that corresponds to the face fan of $F_{4}$. In terms of homogeneous coordinates the ambient space consists of the points

$$
\left[X: Y: Z: e_{1}\right] \in \mathbb{C}^{4} \backslash\left(\{X=Y=0\} \cup\left\{e_{1}=Z=0\right\}\right),
$$

that are identified under the equivalence relation

$$
\left[X: Y: Z: e_{1}\right] \sim\left[\lambda_{1} X: \lambda_{1}^{-1} \lambda_{2}^{-2} Y: \lambda_{2} Z: \lambda_{2}^{-1} e_{1}\right],
$$

for all $\lambda_{1}, \lambda_{2} \in \mathbb{C}^{*}$. We will denote this space as $\mathbb{P}_{F_{4}}$ which is in line with the conventions of $[85] .{ }^{13}$ A generic section of the anti-canonical line bundle takes the form

$$
\begin{aligned}
p_{F_{4}}= & e_{1}^{2}\left(d_{1} X^{4}+d_{2} X^{3} Y+d_{3} X^{2} Y^{2}+d_{4} X Y^{3}+d_{5} Y^{4}\right) \\
& +e_{1}\left(d_{6} X^{2}+d_{7} X Y+d_{8} Y^{2}\right) Z+d_{9} Z^{2} .
\end{aligned}
$$

\footnotetext{
${ }^{13}$ Note that this is at odds with e.g. [135] where the name $\mathbb{P}_{F_{13}}$ would have been used.
} 
At this level the coefficients $d_{i}, i=1, \ldots, 9$ are complex numbers and redundantly parametrize the complex structure of the elliptic curve $\left\{p_{F_{4}}=0\right\} \subset \mathbb{P}_{F_{4}}$. We can construct a genus one fibration over a base $B$ by choosing four line bundles of which $X, Y, Z, e_{1}$ are taken to be sections. The requirement that $p_{F_{4}}$ is also a section of a well-defined bundle and that $X=\left\{p_{F_{4}}=0\right\}$ is a Calabi-Yau manifold then also fixes the bundles of which the coefficients have to be sections. In fact, the choice of four bundles is redundant since the equivalence relation (7.4) can be used to let e.g. $X^{-1} Z$ and $e_{1}$ be sections of the trivial bundle. Again following the conventions laid out in [85] we pick two line bundles $\mathcal{S}_{7}, \mathcal{S}_{9}$ on $B$ and fix the classes

$$
\begin{array}{rlrl}
{[X]} & =H-E_{1}+\mathcal{S}_{9}-c_{1}(B), & & {[Y]=H-E_{1}-\mathcal{S}_{7}+\mathcal{S}_{9},} \\
{[Z]=2 H-E_{1}+\mathcal{S}_{9}-c_{1}(B),} & & {\left[e_{1}\right]=E_{1},}
\end{array}
$$

for the toric divisors of $\mathbb{P}_{F_{4}}$.

The class of coefficients. The Calabi-Yau requirement imposes

$$
\left[p_{F_{4}}\right]=c_{1}(B)+c_{1}\left(\mathbb{P}_{F_{4}}\right)=c_{1}(B)+[X]+[Y]+[Z]+\left[e_{1}\right],
$$

and therefore fixes the class of the coefficient $\left[d_{7}\right]=c_{1}(B)$ that corresponds to the unique inner point of $F_{13}$. It is now easy to see that the charge of the coefficient that multiplies a monomial corresponding to any $m \in F_{13}$ is given by

$$
\left[d_{m}\right]=c_{1}(B)+\sum_{\rho \in \Sigma(1)}\langle m, \rho\rangle\left[D_{\rho}\right]_{B} .
$$

Here we introduced the notation $D_{\rho}$ for the divisor that corresponds to the generator $\rho \in \Sigma(1)$ and $[D]_{B}$ for the base part of the class of a divisor. Inserting the classes of $[X],[Y],[Z]$ and $\left[e_{1}\right]$ this determines

$$
\begin{aligned}
& {\left[d_{1}\right]=3 c_{1}(B)-\mathcal{S}_{7}-\mathcal{S}_{9}, \quad\left[d_{2}\right]=2 c_{1}(B)-\mathcal{S}_{9}, \quad\left[d_{3}\right]=c_{1}(B)+\mathcal{S}_{7}-\mathcal{S}_{9},} \\
& {\left[d_{4}\right]=2 \mathcal{S}_{7}-\mathcal{S}_{9}, \quad\left[d_{5}\right]=-c_{1}(B)+3 \mathcal{S}_{7}-\mathcal{S}_{9}, \quad\left[d_{6}\right]=2 c_{1}(B)-\mathcal{S}_{7},} \\
& {\left[d_{7}\right]=c_{1}(B), \quad\left[d_{8}\right]=\mathcal{S}_{7}, \quad\left[d_{9}\right]=c_{1}(B)-\mathcal{S}_{7}+\mathcal{S}_{9} .}
\end{aligned}
$$

From now on we will assume that the base $B$ is itself a smooth toric variety. The line bundles on $B$ are then determined by charge vectors in terms of a basis of effective divisors. Let us assume that the cone of effective divisors is generated by $k$ independent classes and therefore the charges are elements $\vec{q} \in \mathbb{Z}^{k}$. We will also assume that the cone is simplicial i.e. $k$-dimensional although this assumption can be easily dropped. In any case, (7.8) can now be read component wise. If we demand that every coefficient $d_{i}, i=1, \ldots, 9$ appears in $p_{F_{4}}$ this leads to $k$ inequalities

$$
q_{j}\left(\left[d_{m}\right]\right)=q_{j}\left(c_{1}(B)+\sum_{\rho \in \Sigma(1)}\langle m, \rho\rangle\left[D_{\rho}\right]_{B}\right) \geq 0, \quad j=1, \ldots, k,
$$

in terms of the $k$-th charges of $\mathcal{S}_{7}$ and $\mathcal{S}_{9}$ for every monomial $m \in F_{12}$. The inequalities define $k$ polytopes in $k\left(q_{i}\left(\mathcal{S}_{7}\right), q_{i}\left(\mathcal{S}_{9}\right)\right)$-planes and each one is related via a lattice automorphism to $q_{i}\left(c_{1}(B)\right) \cdot F_{4}$. 

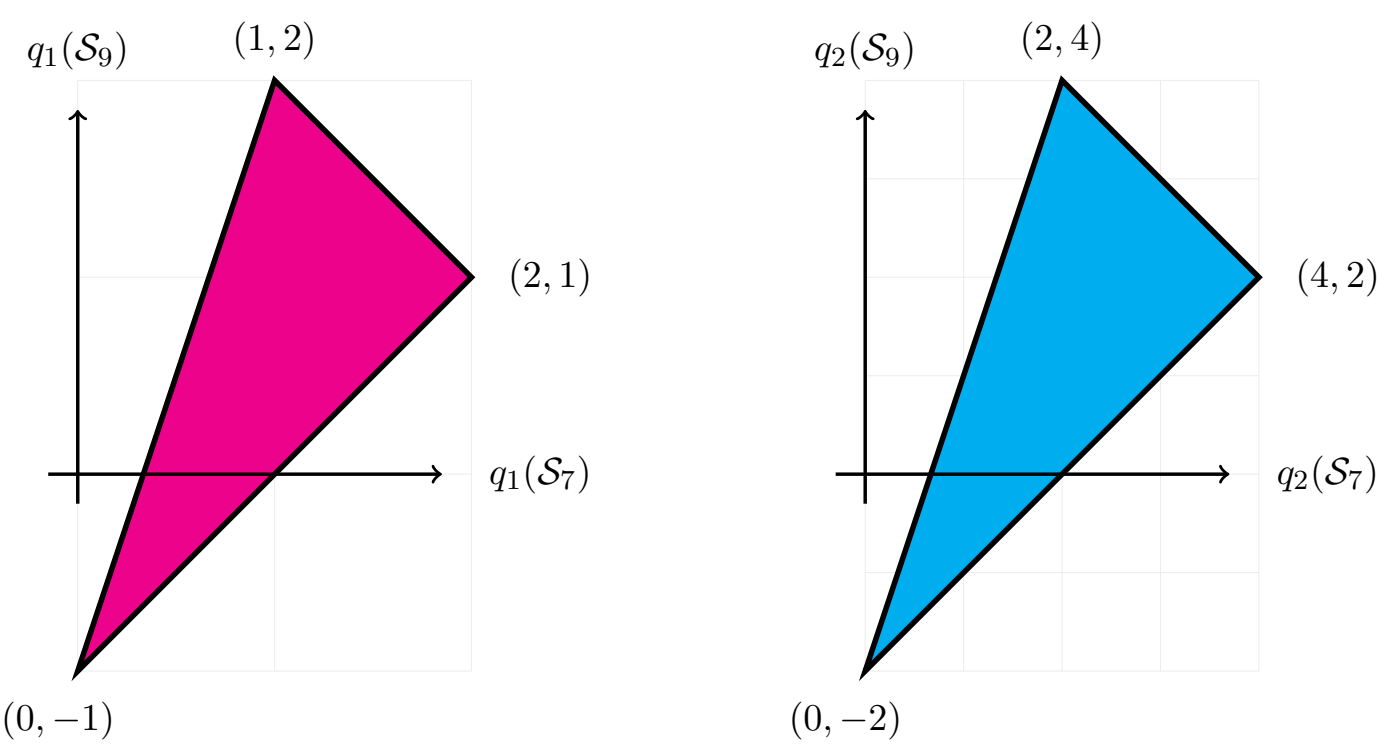

Figure 6. The admissible choices for $\left(q_{i}\left(\mathcal{S}_{7}\right), q_{i}\left(\mathcal{S}_{9}\right)\right), i=1,2$ correspond to polytopes that are related via a lattice automorphism and scaling to $F_{4}$.

Note that if we allow some of the coefficients to generically vanish we might as well work with the toric ambient space that is associated to the dual of the Newton polytope of the non-vanishing monomials. Due to (7.8) it is not possible that the hull of the set of points that correspond to coefficients in an effective class is non-convex. We can therefore impose (7.10) wihout loss of generality.

Example $\boldsymbol{B}=\mathbb{F}_{\mathbf{1}}$. To make this somewhat abstract discussion more concrete we illustrate it for $B=\mathbb{F}_{1}=\mathbb{P}_{F_{3}}$. This can be constructed as the quotient

$$
\left[u: v: w: e_{1}\right] \in \frac{\mathbb{C}^{4} \backslash\left(\{v=u=0\} \cup\left\{w=e_{1}=0\right\}\right)}{\sim},
$$

where the equivalence relations are

$$
\left[u: v: w: e_{1}\right] \sim\left[\lambda_{1} \lambda_{2}^{-1} u: \lambda_{2} v: \lambda_{1}^{-1} w: \lambda_{1} e_{1}\right]
$$

for all $\lambda_{1}, \lambda_{2} \in \mathbb{C}^{*}$. The cone of effective divisors on $B$ is generated by $u$ and $w$ and the charge of the first Chern class with respect to this basis is $\vec{q}\left(c_{1}(B)\right)=(1,2)$. The solutions to the inequalities (7.10) are the points in the shaded regions shown in figure 6 .

The properties of the resulting fibrations for a generic choice of $\mathcal{S}_{7}, \mathcal{S}_{9}$ have been summarized in [85]. If one chooses $\mathcal{S}_{7}, \mathcal{S}_{9}$ such that the charge is on the boundary of both polytopes the class of some of the coefficients $d_{i}$ will be trivial. It is still easy to deduce the resulting properties from the generic expressions that have been provided in [85]. In particular the sections, multi-sections and singularities of the fibrations in various codimensions have been determined for all hypersurfaces that correspond to the 16 two-dimensional reflexive polytopes. A powerful strategy is therefore to select a fiber-polytope that leads to the desired gauge group via F-theory of which the non-Abelian part can be further broken down with a particular choice of $\mathcal{S}_{7}, \mathcal{S}_{9}$. We will now review how for any such choice of fiber, toric base and bundles one can recover the reflexive polytope of the total ambient space. 


\begin{tabular}{|c|cccc|}
\hline & $X$ & $Y$ & $Z$ & $e_{1}$ \\
$q_{1}$ & 1 & 1 & 1 & 0 \\
$q_{2}$ & 0 & -1 & 0 & 0 \\
\hline
\end{tabular}

Table 4. Charges of the homogeneous coordinates on $\mathbb{P}_{F_{4}}$ with respect to the basis of effective divisors on $B=\mathbb{F}_{1}$ given by $u, w$ for the choice $\mathcal{S}_{7}=(1,3)$ and $\mathcal{S}_{9}=(2,2)$.

Recovering the polytope. Again we provide an example that can easily be generalized. Let us stick to $\mathbb{P}_{F_{4}}$ as the ambient space of the fiber and $B=\mathbb{F}_{1}=\mathbb{P}_{F_{3}}$ as the base. A generic choice of bundles would be $\mathcal{S}_{7}=(1,3)$ and $\mathcal{S}_{9}=(2,2)$. From (7.4) and (7.12) can deduce that this corresponds to the relations

$$
\begin{aligned}
& {\left[X: Y: Z: e_{1}: u: v: w: e_{1}^{\prime}\right] } \\
\sim & {\left[\lambda^{l_{1}^{(i)}} X: \lambda^{l_{2}^{(i)}} Y: \lambda^{l_{3}^{(i)}} Z: \lambda^{l_{4}^{(i)}} e_{5}: \lambda^{l_{6}^{(i)}} u: \lambda^{l_{7}^{(i)}} v: \lambda^{l_{8}^{(i)}} w: \lambda^{l_{9}^{(i)}} e_{1}^{\prime}\right], }
\end{aligned}
$$

for all $\lambda \in \mathbb{C}^{*}$ and $i=1, \ldots, 4$, where

$$
\begin{aligned}
& l^{(1)}=\left(\begin{array}{l}
1, \quad 1,1, \quad 0,1,1,0,-1
\end{array}\right), \\
& l^{(2)}=(0,-1,0, \quad 0,0,0,1, \quad 1), \\
& l^{(3)}=\left(\begin{array}{lll}
1, & 1,0,-2,0,0,0, & 0
\end{array}\right), \\
& l^{(4)}=\left(\begin{array}{lll}
0, & 0,1, \quad 1,0,0,0, & 0
\end{array}\right) .
\end{aligned}
$$

The last four entries of $l^{(1)}, l^{(2)}$ form a basis of charges for the equivalence relations that define $\mathbb{F}_{1}$ such that $u$ has charge 1 under $l^{(1)}$ and charge 0 under the relation $l^{(1)}$ while the opposite holds for $w$. This implies that the first four entries of $l^{(i)}, i=1,2$ consist of the charges $q_{i}(X), q_{i}(Y), q_{i}(Z), q_{i}\left(e_{1}\right)$ that are listed in table 4 . Those can in turn be obtained from $q_{i}\left(\mathcal{S}_{7}\right)$ and $q_{i}\left(\mathcal{S}_{9}\right)$ via equation (7.6). The relations $l^{(3)}$ and $l^{(4)}$ directly correspond to relations among the fiber coordinates (7.4).

Let us denote the $8 \times 4$ matrix of relations as $Q$, i.e. $Q_{i j}=l_{i}^{(j)}$. Note that the exponents of the scaling relations among the homogeneous coordinates of a toric variety directly correspond to coefficients in linear relations among the points that generate the one-dimensional cones $\Sigma(1)$ of the fan. One can often obtain a reflexive polytope from the set of relations by considering the kernel

$$
V=\operatorname{ker}\left(Q^{t}\right)
$$

and then finding a basis of the sublatttice $V \cap \mathbb{Z}^{8}$. We now take the elements of such a basis as the columns of a matrix

$$
A=\left(\begin{array}{rrrr}
1 & 0 & 0 & 0 \\
-1 & 1 & 0 & 0 \\
-1 & -1 & 0 & 0 \\
0 & 0 & 1 & 0 \\
0 & 0 & 0 & 1 \\
0 & 1 & -1 & -1 \\
-1 & 1 & -1 & 0
\end{array}\right)
$$




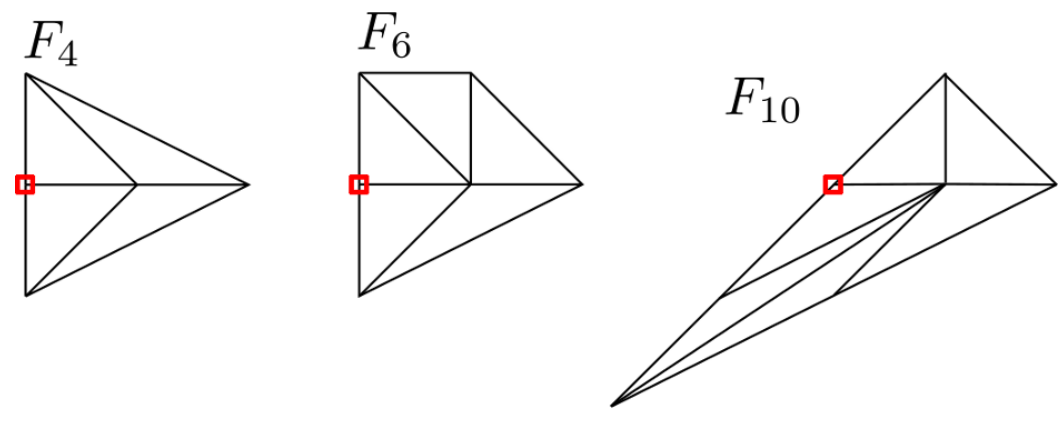

Figure 7. Fibers of the Higgs chain $\mathbb{Z}_{2} \leftarrow \mathrm{U}(1) \leftarrow \mathrm{SU}(2)$.

The rows of $A$ determine a set of points in $\mathbb{Z}^{4}$ and the convex hull is the desired reflexive polytope $\Delta$. This algorithm to obtain the polytope from a basis of relations has been reviewed e.g. in [136].

Looking at the points in (7.16) we see that there is a 2-dimensional sublattice $L$ that intersects $\Delta$ as $L \cap \Delta=F_{4}$. On the other hand, projection on the last two coordinates recovers the polytope $F_{3}$ that correpsonds to the base of the fibration. In fact this structure is necessary for the corresponding toric variety to be an $\mathbb{P}_{F_{4}}$ fibration over $\mathbb{P}_{F_{3}}$.

\subsection{The Higgs chain $\mathrm{SU}(2) \rightarrow \mathrm{U}(1) \rightarrow \mathbb{Z}_{2}$}

As our first set of examples we study the Higgs chain that is indicated in figure 7 where the base for all of the fibrations is $B=\mathbb{P}^{2}$. At the bottom of the chain we start with a genus one fibered Calabi-Yau threefold $M_{1}^{h}=\left(F_{4} \rightarrow \mathbb{P}^{2}\right)\left[\mathbb{Z}_{2}\right]_{2}^{-252}$ that only admits a two-section and no fibral divisors.

This is related via an extremal transition to the elliptic fibration $M_{2}^{h}=\left(F_{6} \rightarrow\right.$ $\left.\mathbb{P}^{2}\right)[\mathrm{U}(1)]_{3}^{-216}$ that has two independent sections. In particular, the corresponding F-theory effective action exhibits hypermultiplets of charge one and two. In geometric terms the charge two matter arises from fibral curves that are wrapped by the section that generates the Mordell-Weil group and are intersected transversely by the zero section.

On a subslice of the complex structure moduli space of $M_{2}^{h}$ some of the isolated fibral curves deform into families of $I_{2}$ fibers over a genus $g=10$ Riemann surface in the base. This enhances the Abelian gauge group of the corresponding F-theory vacuum into SU(2). Other fibral curves remain isolated and lead to $n=72$ hypermultiplets in the fundamental representation. Note that the number of the charge two loci in $M_{2}^{h}$ is $2 g-2=18$ while the number of charge one loci is $2 n=144$.

We construct this subslice as a hypersurface in a toric ambient space $M_{3}^{h}=\left(F_{10} \rightarrow\right.$ $\left.\mathbb{P}^{2}\right)[\mathrm{SU}(2)]_{3}^{-216}$ where $g=10$ complex structure deformations are non-polynomial. The discriminant becomes reducible and on one component the fibral divisor collapses to a curve in the base.

\subsection{1 $M_{1}^{h}=\left(F_{4} \rightarrow \mathbb{P}^{2}\right)\left[\mathbb{Z}_{2}\right]_{2}^{-252}$}

The fiber of $M_{1}^{h}$ is a hypersurface in $\mathbb{P}_{F_{4}}$ and the base remains $\mathbb{P}^{2}$. Choosing the line bundles $\mathcal{S}_{7}=3 H, \mathcal{S}_{9}=0$ with $H$ being the class of the hyperplane in $\mathbb{P}^{2}$ leads to the toric 
data given in (7.17),

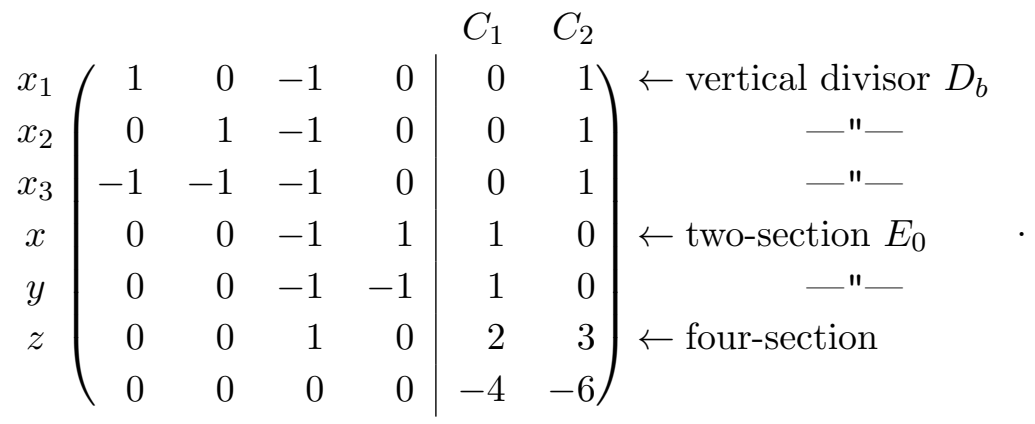

The relevant points of the polytope admit two different regular fine star triangulations. Only one of the triangulations is compatible with the toric morphism that induces the fibration. But the curve that is flopped when moving from one geometric phase to the other is not contained in the generic Calabi-Yau hypersurface. Therefore the flop that connects the two phases is ineffective and we should work with the intersection of the Mori cones. The latter is generated by the curves $C_{1}$ and $C_{2}$ that are intersecting the toric divisors as listed in (7.17).

The geometry of the image of the toric divisors on the Calabi-Yau is as follows. As usual, $D_{b}$ is the class of the pullback of the hyperplane in $\mathbb{P}^{2}$. In addition there are two equivalent two-sections $\{x=0\}$ and $\{y=0\}$ with $E_{0}=[x]=[y]$ and a four section $\{z=0\}$. The class of the generic fiber is $C_{1}^{\prime}=D_{b}^{2}$ while $C_{2}^{\prime}=E_{0} \cdot D_{b}$ is the restriction of the two-section to the generator of the Mori cone of the base. The latter is a double cover of a $\mathbb{P}^{1}$. It is clear that $E \cdot C_{1}^{\prime}=D_{b} \cdot C_{2}^{\prime}=2$.

But the generic fiber is in fact not a generator of the Mori cone of $M_{1}^{h}$. There are isolated $I_{2}$ singular fibers over 144 points of $B$ and the two-sections intersect each of the two components once [85]. Physically, this is a consequence of charge minimality and the fact that the discrete symmetry that corresponds to the multi-section has to arise from a Higgsed U(1) gauge symmetry. After this Higgsing, the 144 hypermultiplets with discrete charge $q=1$ are the remnants of the same number of hypermultiplets that are minimally charged under the U(1). We will study the un-Higgsed geometry below.

To fix the numerator of the topological string partition function for the lowest base degree, let us calculate the Gopakumar-Vafa invariants at genus zero and genus one. To this end we choose a basis $J_{1}=E_{0}, J_{2}=D_{b}$ of the Kähler cone such that

$$
\int_{C_{i}^{\prime}} J_{j}=2 \delta_{i, j},
$$

and use it to parametrize the complexified Kähler form as

$$
\omega=t^{1} J_{1}+t^{2} J_{2} .
$$

Note that $t^{1}$ is the volume of each component of the isolated $I_{2}$ fibers while $t^{2}$ is half of the volume of $C_{2}^{\prime}$. It turns out that this is the correct parametrization to obtain integer 
Gopakumar-Vafa invariants. The topological invariants (3.12) are

$$
h^{1,1}=2, \quad \chi=-252, \quad \vec{b}=\int_{M_{1}^{h}} c_{2}\left(M_{1}^{h}\right) \cdot \vec{J}=(24,36)^{T},
$$

and the only non-vanishing triple intersection number is $c_{122}=2$. With this data the genus zero free energy can then be obtained e.g. using the techniques reviewed in [137].

To obtain the genus one free energy we will briefly discuss the mirror geometry $W$. The polynomial that determines $W$ in the dual ambient space is of the form

$$
\begin{aligned}
P= & x_{1}^{2}+\left(x_{3} x_{4} x_{5}\right)^{4}+z_{1}\left(x_{2} x_{6} x_{7}\right)^{4}+z_{2}\left(x_{5} x_{6}\right)^{6} \\
& +\left(x_{2} x_{3}\right)^{6}+\left(x_{4} x_{7}\right)^{6}+x_{1} x_{2} x_{3} x_{4} x_{5} x_{6} x_{7} .
\end{aligned}
$$

Here the coordinates $z_{1}, z_{2}$ on the complex structure moduli space are identified via mirror symmetry with $q_{i}+\mathcal{O}\left(q^{2}\right)$, where $q_{i}=e^{2 \pi i t^{i}}$. The Picard-Fuchs system is generated by the operators

$$
\begin{aligned}
& \mathcal{D}_{1}=\Theta_{1}^{2}-4 z_{1}\left(4 \Theta_{1}+6 \Theta_{2}+1\right)\left(4 \Theta_{1}+6 \Theta_{2}+3\right), \\
& \mathcal{D}_{2}=\Theta_{2}^{3}-8 z_{2}\left(4 \Theta_{1}+6 \Theta_{2}+1\right)\left(4 \Theta_{1}+6 \Theta_{2}+3\right)\left(4 \Theta_{1}+6 \Theta_{2}+5\right) .
\end{aligned}
$$

The discriminant is irreducible and corresponds to the vanishing locus of the polynomial

$$
\Delta=1-192 z_{1}+12288 z_{1}^{2}-262144 z_{1}^{3}-3456 z_{2}-663552 z_{1} z_{2}+2985984 z_{2}^{2} .
$$

We then find that the ansatz

$$
\begin{aligned}
F_{1}= & -\frac{1}{2}\left(3+h^{1,1}-\frac{\chi}{12}\right) K-\frac{1}{2} \log \operatorname{det} G^{-1} \\
& -\frac{1}{24} \sum_{i=1}^{2}\left(b_{i}+12\right) \log z^{i}-\frac{1}{12} \log \Delta,
\end{aligned}
$$

for the free energy at genus one leads to integer Gopakumar-Vafa invariants.

Let us now discuss the "modular parametrization" of the Kähler form. Following our discussion in 3.3 , we calculate

$$
E_{0} \cdot C_{2}^{\prime}=0
$$

and therefore $\tilde{E}_{0}=E_{0}$. This also implies that

$$
\tilde{a}_{b}=\tilde{E}_{0}^{2} \cdot D_{b}=0 .
$$

We therefore parametrize the Kähler form as

$$
\omega=\tau \cdot E_{0}+t \cdot D_{b} .
$$

If we assume that poles in $q$ are always cancelled we can write down the general ansatz

$$
Z_{d}(\tau, \lambda)=\frac{\Delta_{4}^{3 d}}{\eta(2 \tau)^{36 d}} \frac{\phi_{d}(\tau, \lambda)}{\prod_{k=1}^{d} \phi_{-2,1}(2 \tau, k \lambda)},
$$




\begin{tabular}{|c|cccc|}
\hline$d_{F} \backslash g$ & 0 & 1 & 2 & 3 \\
\hline 0 & 2496 & 0 & 0 & 0 \\
1 & 216576 & 0 & 0 & 0 \\
2 & 6391296 & -4992 & 0 & 0 \\
3 & 104994816 & -433152 & 0 & 0 \\
4 & 1209337344 & -12797568 & 7488 & 0 \\
5 & 10917983232 & -211289088 & 649728 & 0 \\
6 & 82279299072 & -2457042432 & 19213824 & -9984 \\
7 & 538501165056 & -22467667968 & 318449664 & -866304 \\
\hline
\end{tabular}

Table 5. Gopakumar-Vafa invariants for $\left(F_{4} \rightarrow \mathbb{P}^{2}\right)\left[\mathbb{Z}_{2}\right]_{2}^{-252}$ at base degree 1 .

where $\phi_{d}$ is a Jacobi form of weight $4 d$ and index

$$
\tilde{r}_{\lambda}^{d}=\frac{1}{4} d(d-3)+\frac{1}{2} \sum_{k=1}^{d} k^{2}=\frac{1}{6} d(d-1)(d+4) .
$$

Together with the dimensions of the spaces of modular forms for $\Gamma_{1}(2)$,

$$
\operatorname{dim} M_{w}(2)=\left\lfloor\frac{w}{4}\right\rfloor+1
$$

one find that there are

$$
\alpha_{d}=\tilde{r}_{\lambda}^{d}+\left(\tilde{r}_{\lambda}^{d}+1\right) d+\left\lfloor\frac{1}{4} \tilde{r}_{\lambda}^{d}\left(\tilde{r}_{\lambda}^{d}+1\right)\right\rfloor,
$$

coefficients that determine $\phi_{d}$. Using the Gopakumar-Vafa invariants at genus zero and genus one we can fix

$$
\phi_{1}(\tau, \lambda)=192\left[12\left(E_{2}^{(2)}\right)^{2}+E_{4}\right] .
$$

To obtain the partition function at base degree two we have to make some assumption about the vanishing of Gopakumar-Vafa invariants. Some invariants at base degree one that can be extracted from (7.32) are shown in table 5 and one can observe that $n_{d_{F}, 1}^{g}$ vanishes for $d_{F}<2 g$. It turns out that this does not hold for $d_{B}>1$ but at $d_{B}=2$ we can impose that $n_{d_{F}, 1}^{g}$ vanishes for $d_{F}<2\lfloor g / 2\rfloor$. This fixes

$$
\begin{aligned}
\phi_{2}(\tau, \lambda)= & \frac{32}{9} A^{4} \cdot\left(12 g^{2}+h\right)^{2}+A^{3} B \frac{4}{27} g\left(1072 g^{4}-7832 g^{2} h-797 h^{2}\right) \\
& -\frac{1}{54} A^{2} B^{2} \cdot\left(4 g^{2}-h\right)\left(25504 g^{4}+6924 g^{2} h+227 h^{2}\right) \\
& +A B^{3} \cdot \frac{g\left(1425683 g^{6}+7311527 g^{4} h-733303 g^{2} h^{2}-154563 h^{3}\right)}{1728} \\
& +B^{4} \cdot \frac{2550099 g^{8}-20848992 g^{6} h+2131870 g^{4} h^{2}+885304 g^{2} h^{3}+8887 h^{4}}{6912},
\end{aligned}
$$

where we introduce

$$
A=\phi_{0,1}(2 \tau, \lambda), \quad B=\phi_{-2,1}(2 \tau, \lambda), \quad g=E_{2}^{(2)}(\tau), \quad h=E_{4}(\tau) .
$$




\begin{tabular}{|c|c|c|c|c|c|}
\hline$d_{F} \backslash g$ & 0 & 1 & 2 & 3 & 4 \\
\hline 0 & 223752 & -492 & 0 & 0 & 0 \\
\hline 1 & 152031744 & 69120 & 0 & 0 & 0 \\
\hline 2 & 19638646848 & 104982288 & -304464 & -1476 & 0 \\
\hline 3 & 1180450842624 & 11531535360 & -214941696 & 207360 & 0 \\
\hline 4 & 43199009739072 & 582562932240 & -23399572104 & 308769960 & -601056 \\
\hline 5 & 1107266933984256 & 18197544339456 & -1223655651840 & 35175416832 & -430989312 \\
\hline 6 & 21665294606886144 & 403387081306944 & -40593035175168 & 1888636322256 & -48423847008 \\
\hline 7 & 342620943505772544 & 6879702812129280 & -975207871309824 & 64188987386880 & -2630615021568 \\
\hline
\end{tabular}

Table 6. Gopakumar-Vafa invariants for $\left(F_{4} \rightarrow \mathbb{P}^{2}\right)\left[\mathbb{Z}_{2}\right]_{2}^{-252}$ at base degree 2 .

\subsection{2 $M_{2}^{h}=\left(F_{6} \rightarrow \mathbb{P}^{2}\right)[\mathrm{U}(1)]_{3}^{-216}$}

The geometry $M_{1}^{h}$ is related via an extremal transition to what we call $M_{2}^{h}$. The choice of line bundles in the conventions of [85] is still $\mathcal{S}_{7}=3 H, \mathcal{S}_{9}=0$ and the toric data is given in $(7.35)$,

$$
\begin{aligned}
& \begin{array}{lll}
C_{1} & C_{2} & C_{3}
\end{array}
\end{aligned}
$$

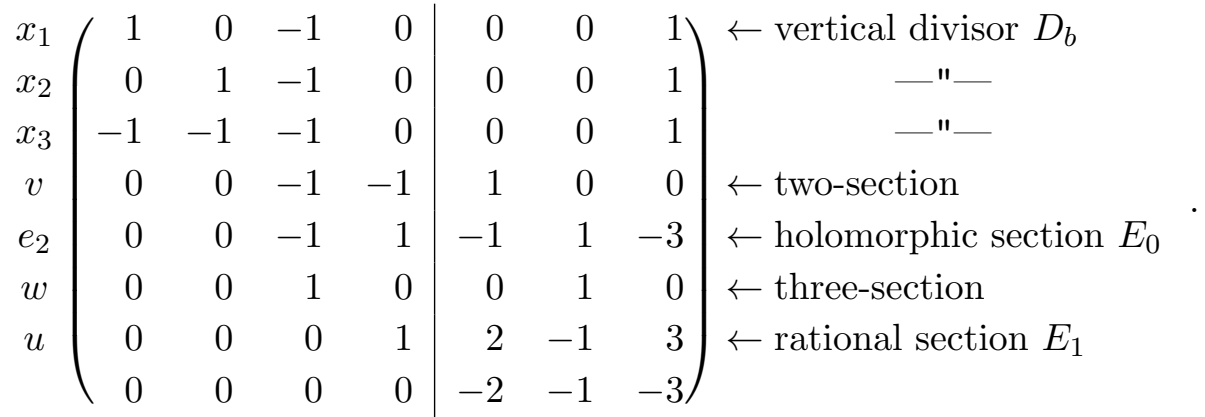

Again we encounter the situtation that the relevant points admit two different regular fine star triangulations. Only one is compatible with the fibration of the ambient space but the phase boundary is lifted by the hypersurface equation. The curves $C_{i}, i=1, \ldots, 3$ in (7.35) generate the intersection of the two Mori cones.

The geometry of the images of the toric divisors on $M_{2}^{h}$ is as follows. The divisor $D_{b}=$ $\left[x_{1}\right]=\left[x_{2}\right]=\left[x_{3}\right]$ is again the pullback of the hyperplane class of the base. Futhermore, $e_{2}=0$ is a holomorphic section while $u=0$ is a linearly independent rational section. We denote the corresponding divisors by $E_{0}=\left[e_{2}\right]$ and $E_{1}=[u]$. That $u=0$ is not holomorphic manifests itself in the fact that it wraps a curve in the fiber over 18 points of the base. ${ }^{14}$ Each of these curves is transversely intersected by $e_{2}=0$. On the other hand $[v]=E_{1}+E_{0}$ intersects the generic fiber twice and $[w]=[v]+E_{0}+3 D_{b}$ intersects it thrice.

Let us now study the Mori cone of $M_{2}^{h}$ directly. It is generated by four curves. The first, $C_{b}^{1}=E_{0} \cdot D_{b}$, is the restriction of the holomorphic section to the generator of the Mori cone of the base. The second, $C_{b}^{2}=E_{1} \cdot D_{b}$, is the restriction of the rational section. The other two curves arise from resolutions of singularities in the fiber. Over 18 points in the

\footnotetext{
${ }^{14}$ This can easily be determined by studying the hypersurface equation of $M$ or, even easier, using the general results from [85].
} 
base the fiber develops an $I_{2}$ singularity that leads to matter with charge $q=2$. Another $144 I_{2}$ singularities lead to matter with charge $q=1$. In the resolved geometry there are two spheres $C_{A}^{q}, C_{B}^{q}$ over each point and the respective numerical equivalence class only depends on the charge of the matter from that locus. The intersections are as follows:

$$
\begin{array}{r|rrrrrr} 
& C_{A}^{1} & C_{B}^{1} & C_{A}^{2} & C_{B}^{2} & C_{b}^{1} & C_{b}^{2} \\
\hline E_{0} & 0 & 1 & 1 & 0 & 3 & -3 \\
E_{1} & 1 & 0 & -1 & 2 & -3 & 3 \\
D_{b} & 0 & 0 & 0 & 0 & 1 & 1
\end{array}
$$

From this we see that $C_{b}^{1}, C_{b}^{2}, C_{A}^{1}, C_{A}^{2}$ generate the Mori cone on $M_{2}^{h}$.

This being said we will introduce $J_{1}=[v], J_{2}=[w], J_{3}=D_{b}$ and expand the complexified Kähler form as

$$
\omega=t^{1} \cdot J_{1}+t^{2} \cdot J_{2}+t^{3} \cdot J_{3}
$$

where we have replaced the basis element $E_{0}+3 D_{b}$ with the element $[w]$ from the interior of the Kähler cone. This enables us to apply the usual machinery to calculate GopakumarVafa invariants of toric hypersurfaces.

The topological invariants (3.12) are

$$
h^{1,1}=3, \quad \chi=-216, \quad \vec{b}=\int_{M_{2}^{h}} c_{2}\left(M_{2}^{h}\right) \cdot \vec{J}=(24,126,36)^{T},
$$

and the non-vanishing triple intersection numbers are encoded in the polynomial

$$
\mathcal{J}=63 J_{2}^{3}+18 J_{1} J_{2}^{2}+15 J_{3} J_{2}^{2}+3 J_{3}^{2} J_{2}+6 J_{1} J_{3} J_{2}+2 J_{1} J_{3}^{2} .
$$

The section that determines the mirror $W$ in the dual ambient space is given by

$$
\begin{aligned}
P= & -z_{3} \cdot x_{2}^{6} x_{3}^{6} x_{4}^{3}-z_{2} \cdot x_{1}^{2} x_{4} x_{5} x_{6}+z_{1} \cdot x_{2}^{3} x_{4}^{2} x_{5}^{2} x_{6}^{2} x_{7}^{3} x_{8}^{3}+x_{10}^{6} x_{5}^{3} x_{8}^{6} \\
& +x_{1} x_{2} x_{3} x_{4} x_{5} x_{6} x_{7} x_{8} x_{9} x_{10}+x_{1} x_{10}^{3} x_{3}^{3} x_{9}^{3}+x_{10}^{4} x_{2} x_{3}^{4} x_{7} x_{8} x_{9}^{4}+x_{6}^{3} x_{7}^{6} x_{9}^{6},
\end{aligned}
$$

where $z_{i}, i=1, \ldots, 3$ are the Batyrev coordinates on the complex structure moduli space. The Picard-Fuchs system is comparatively simple and given in the appendix C.1. When expressed in terms of the Batyrev variables the discriminant $\Delta$ is irreducible and also given in C.1. The logarithm of $\Delta$ contributes to the genus one free energy with the usual factor of $-1 / 12$.

We will now discuss the Shioda map and the height pairing. Let us fix $\left\{e_{2}=0\right\}$ as the holomorphic zero section and $\{u=0\}$ as the generator of the Mordell-Weil group. Then the image of the generator under the Shioda map is given by

$$
\sigma(\{u=0\})=E_{1}-E_{0}-2 \cdot \pi^{*} c_{1}(B)=3 J_{1}-2 J_{2},
$$

where we have used that $\pi^{*} \pi_{*}\left(E_{1} \cdot E_{0}\right)=\pi^{*} c_{1}(B)$. The corresponding height pairing is

$$
b_{11}=-\pi\left(D_{b}\right) \cdot \pi(\sigma(\{u=0\}) \cdot \sigma(\{u=0\}))=12 .
$$


We also calculate

$$
E_{0}^{2} \cdot D_{b}=-3
$$

and therefore introduce

$$
\tilde{E}_{0}=E_{0}+3 D_{b}
$$

with

$$
\tilde{a}_{b}=\tilde{E}_{0}^{2} \cdot D_{b}=3
$$

The correct modular parametrization of the Kähler form is therefore

$$
\begin{aligned}
\omega & =\tau \cdot \tilde{E}_{0}+m \cdot \sigma(\{u=0\})+\tilde{t} \cdot D_{b} \\
& =\tau \cdot\left(-J_{1}+J_{2}\right)+m \cdot\left(3 J_{1}-2 J_{2}\right)+\tilde{t} \cdot J_{3},
\end{aligned}
$$

where $\tau$ is the volume of the generic fiber, $m$ is the volume of the isolated fibral curve $C_{A}^{1}$ and $\tilde{t}$ is the volume of $C_{b}^{1}$ and $C_{b}^{2}$. The new parameters are related to $t_{i}, i=1, \ldots, 3$ via

$$
\tau=2 t^{1}+3 t^{2}, \quad m=t^{1}+t^{2}, \quad \tilde{t}=t^{3} .
$$

The shifted Kähler parameter is the base is $t=\tilde{t}+\frac{3}{2} \tau$. We can then use the GopakumarVafa invariants at genus zero and genus one to obtain

$$
Z_{1}(\tau, m, \lambda)=\frac{1}{\eta(\tau)^{36}} \frac{\phi_{1}(\tau, m)}{\phi_{-2,1}(\tau, \lambda)},
$$

with

$$
\begin{aligned}
\phi_{1}=\frac{1}{2^{16} 2^{7}} & -A^{6} \cdot h\left(31 h^{3}+113 g^{2}\right)-6 A^{5} B \cdot g\left(115 h^{3}+29 g^{2}\right) \\
& -3 A^{4} B^{2} \cdot h^{2}\left(203 h^{3}+517 g^{2}\right)-4 A^{3} B^{3} \cdot h g\left(479 h^{3}+241 g^{2}\right) \\
& -3 A^{2} B^{4}\left(51 h^{6}+581 h^{3} g^{2}+88 g^{4}\right)-6 A B^{5} \cdot h^{2} g\left(19 h^{3}+125 g^{2}\right) \\
& \left.+B^{6} \cdot\left(9 h^{6}-49 h^{3} g^{2}-104 g^{4}\right)\right],
\end{aligned}
$$

where

$$
A=\phi_{0,1}(\tau, m), \quad B=\phi_{-2,1}(\tau, m), \quad h=E_{4}(\tau), \quad g=E_{6}(\tau) .
$$

One can easily check that $Q \cdot Z_{1}(2 \tau, \tau, \lambda)$ matches the result for $Q \cdot Z_{1}(\tau, \lambda)$ from $M_{1}^{h}$.

$$
\text { 7.2.3 } M_{3}^{h}=\left(F_{10} \rightarrow \mathbb{P}^{2}\right)[\mathrm{SU}(2)]_{3}^{-216}
$$

The final geometry in the second Higgs chain describes a 101 dimensional subslice inside the 111 dimensional complex structure moduli space of $M_{2}^{h}$. On this subslice the $2 g-2=18$ isolated $I_{2}$ fibers of $M_{2}^{h}$ that lead to matter with charge $q=2$ under the U(1) deform into a genus $g=10$ curve of $I_{2}$ singularities and therefore the U(1) un-Higgses into an $\mathrm{SU}(2)$ gauge symmetry. The 144 charge $q=1$ multiplets from $M_{2}^{h}$ arrange into 72 hyper 
multiplets in the fundamental representation of $\mathrm{SU}(2)$. Geometrically these arise from $I_{3}$ enhancements of the $I_{2}$ singular fibers over 72 points in the base of $M_{3}^{h}$.

We realize this subslice as a hypersurface in a toric ambient space where $g=10$ complex structure deformations are non-polynomial. The toric data is given in (7.51),

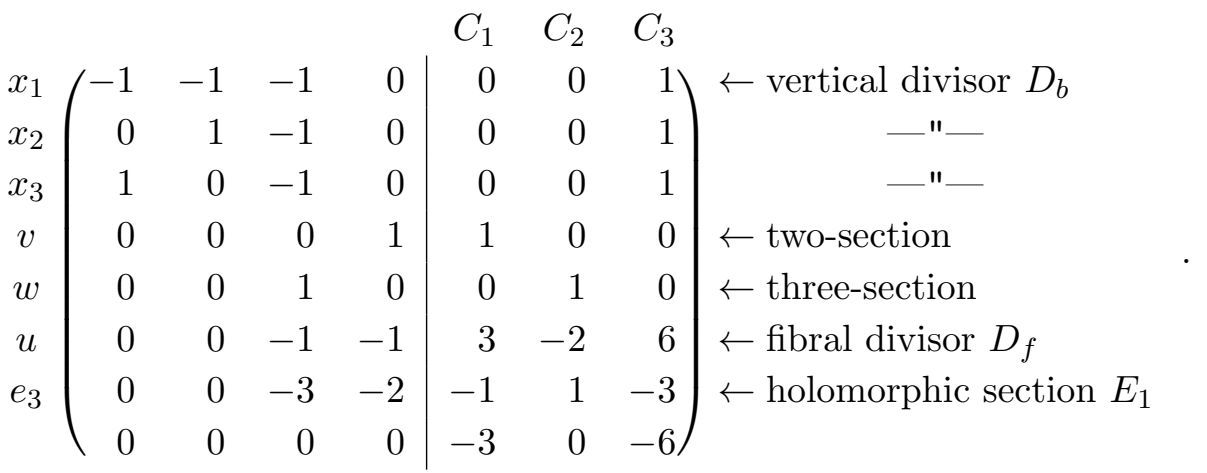

In the conventions of [85] this geometry corresponds to the choice of line bundles $\mathcal{S}_{7}=$ $c_{1}(B), \mathcal{S}_{9}=0$ on $B=\mathbb{P}^{2}$. The curve of $I_{2}$ fibers is in the class $S_{\mathrm{SU}(2)}=2 \mathcal{S}_{7}-\mathcal{S}_{9}=2 c_{1}(B)$. Note that the fibral divisor $D_{f}=[u]$ corresponds to the affine node that is intersected by the holomorphic zero section $E_{1}=\left[e_{3}\right]$. We therefore introduce $D_{f}^{\prime}=S_{\mathrm{SU}(2)}-D_{f}$ to denote the other fibral divisor such that $D_{f}^{\prime} \cdot E_{1}=0$. If we introduce $J_{1}=[v], J_{2}=[w]$ and $J_{3}=\left[x_{1}\right]$ the topological data of $M_{3}^{h}$ is identical to that of $M_{2}^{h}$.

In Batyrev coordinates $z_{i}, i=1, \ldots, 3$ the hypersurface equation that defines the mirror $W$ in the dual ambient space reads

$$
\begin{aligned}
P= & -z_{1} \cdot x_{3}^{3} x_{4}^{3} x_{5}^{3}+z_{2} \cdot x_{1}^{2}+z_{3} \cdot x_{2}^{6} x_{3}^{6}+x_{5}^{6} x_{6}^{6}+x_{4}^{6} x_{7}^{6} \\
& +x_{1} x_{2} x_{3} x_{4} x_{5} x_{6} x_{7}+x_{1} x_{2}^{3} x_{6}^{3} x_{7}^{3}+x_{2}^{6} x_{6}^{6} x_{7}^{6} .
\end{aligned}
$$

Identifying the mirror maps we find that the Batyrev coordinates of $M_{3}^{h}$ can be mapped to those of $M_{2}^{h}$ via

$$
z_{1} \mapsto z_{1}^{\prime} \frac{\left(1+2 z_{2}^{\prime}\right)^{3}}{1+z_{2}^{\prime}}, \quad z_{2} \mapsto z_{2}^{\prime} \frac{1+z_{2}^{\prime}}{\left(1+2 z_{2}^{\prime}\right)^{2}}, \quad z_{3} \mapsto z_{3}^{\prime} \frac{\left(1+2 z_{2}^{\prime}\right)^{6}}{\left(1+z_{2}^{\prime}\right)^{3}}
$$

where $z_{i}^{\prime}, i=1, \ldots, 3$ parametrize the complex structure moduli space of $M_{2}^{h}$.

The discriminant of $W$ is reducible. The principle component $\Delta_{1}$ is again provided in the appendix (C.4) while the other component reads

$$
\Delta_{2}=1-4 z_{2} .
$$

The generators of the Picard-Fuchs system are also provided in (C.3). In this case we did not try to simplify the operators and expect that a more economical choice can be made.

If we expand the Kähler form as

$$
\begin{aligned}
\omega & =\tau \cdot\left(E_{1}+c_{1}(B)\right)+m \cdot D_{f}^{\prime}+\tilde{t} \cdot J_{3} \\
& =\tau \cdot\left(-J_{1}+J_{2}\right)-m \cdot\left(3 J_{1}-2 J_{2}\right)+\tilde{t} \cdot J_{3},
\end{aligned}
$$


where $\tau$ is the volume of the generic fiber, $m$ is twice the volume of the fiber of $D_{f}$ and $C_{B}$ is the volume of $C_{b}=E_{1} \cdot D_{b}$. The new parameters are related to $t_{i}, i=1, \ldots, 3$ via

$$
\tau=2 t_{1}+3 t_{2}, \quad m=-\left(t_{1}+t_{2}\right), \quad \tilde{t}=t_{3} .
$$

The shifted Kähler parameter is the base is again $t=\tilde{t}+\frac{3}{2} \tau$. Since the family $M_{3}^{h}$ corresponds to a subslice in the complex structure moduli space of $M_{2}^{h}$, the topological string partition functions are identical.

\subsection{A genus-one fibrations over $\mathbb{F}_{1}:\left(F_{4} \rightarrow \mathbb{F}_{1}\right)\left[\mathrm{SU}(2) \times \mathbb{Z}_{2}\right]_{4}^{-144}$}

Now we will demonstrate the modular bootstrap for genus one fibrations at the hand of a 2 -section geometry over the Hirzebruch surface $\mathbb{F}_{1}$ that also exhibits a fibral divisor. To engineer this geometry we can again use the fiber polytope $F_{4}$. Following the discussion in 7.1 we choose the bundles

$$
\mathcal{S}_{7}=2 B+4 F, \quad \mathcal{S}_{9}=2 B+3 F,
$$

where $B$ and $F$ are the base and fiber of the Hirzebruch surface. This leads to the following toric data:

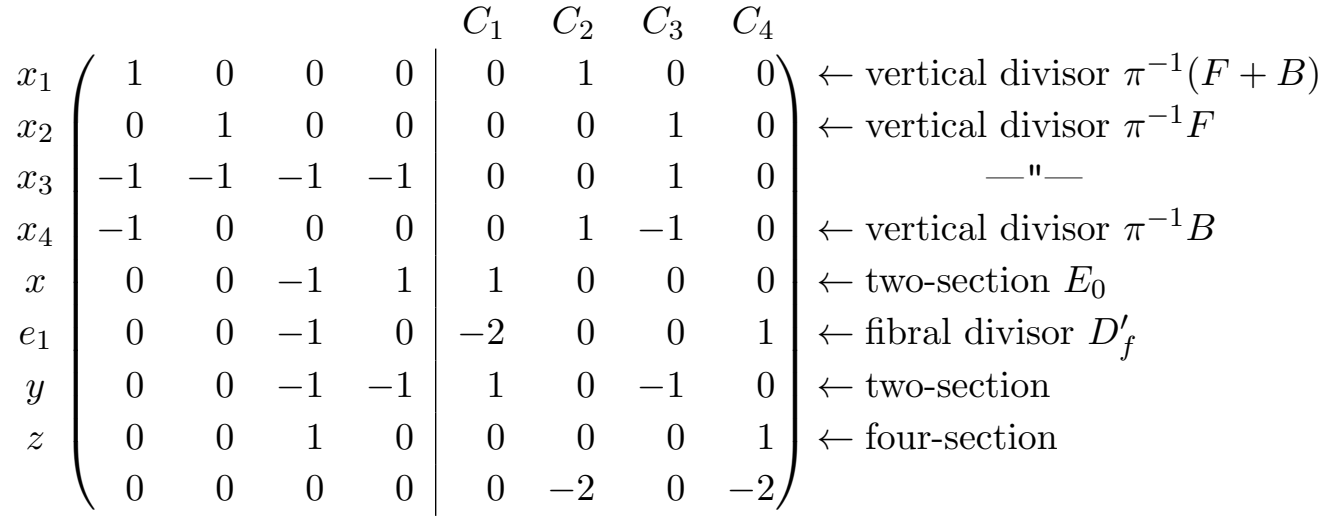

There are three regular fine star triangulations of the relevant points of the polytope and we provided the data for the Mori cone that corresponds to the unique triangulation that is compatible with the fibration.

A convenient basis of divisors to obtain the free energies at genus 0 and genus 1 is

$$
J_{1}=E_{0}, \quad J_{2}=D_{1}^{\prime}, \quad J_{3}=D_{2}^{\prime}, \quad J_{4}=[z]=D_{f}^{\prime}+2 \cdot E_{0} .
$$

The relevant topological invariants are

$$
h^{1,1}=4, \quad \chi=-144, \quad \vec{b}=\int_{M} c_{2}(M) \cdot \vec{J}=(36,36,24,68)^{T},
$$

and the triple intersections are encoded in the polynomial

$$
\begin{aligned}
\mathcal{J}= & 8 J_{4}^{3}+4 J_{1} J_{4}^{2}+12 J_{2} J_{4}^{2}+4 J_{3} J_{4}^{2}+2 J_{1}^{2} J_{4}+4 J_{2}^{2} J_{4}+6 J_{1} J_{2} J_{4} \\
& +2 J_{1} J_{3} J_{4}+4 J_{2} J_{3} J_{4}+2 J_{1} J_{2}^{2}+2 J_{1}^{2} J_{2}+2 J_{1} J_{2} J_{3} .
\end{aligned}
$$


The principal component of the discriminant contains 427 terms and will be provided as supplementary data online [129].

Let us now construct the appropriate parametrization of the Kähler form to perform the modular bootstrap. To this end we introduce

$$
D_{1}^{\prime}=\pi^{-1}(F+B), \quad D_{2}^{\prime}=\pi^{-1}(F), \quad D_{1}=\pi^{-1}(F), \quad D_{2}=\pi^{-1}(B),
$$

and $E_{0}=[x]$ as well as the curves $C_{i}=E_{0} \cdot D_{i}, i=1,2$ such that

$$
D_{i}^{\prime} \cdot C_{j}=2 \cdot \delta_{i j}
$$

Moreover, $\tilde{E}_{0}=E_{0}-D_{1}$ is orthogonal to those curves and we can calculate

$$
\tilde{a}_{i}=\int_{M} \tilde{E}_{0}^{2} \cdot D_{i}=\left\{\begin{array}{rl}
0 & \text { for } i=1 \\
-2 & \text { for } i=2
\end{array} .\right.
$$

From (7.5) and (7.9) we can see that the fibral divisor $D_{f}^{\prime}=\left[e_{1}\right]$ is fibered over a divisor in the class

$$
\left[d_{9}\right]=c_{1}(B)-\mathcal{S}_{7}+\mathcal{S}_{9}=2 B+2 F .
$$

The fibral divisor $D_{f}^{\prime}=\left[e_{1}\right]$ is not orthogonal to $E_{0}$ but we can construct the linear combination of fibral divisors

$$
D_{f}=\frac{1}{2}\left(\left[d_{9}\right]-2 \cdot D_{f}^{\prime}\right)
$$

such that $\tilde{E}_{0} \cdot D_{f} \cdot D_{i}=0$ for $i=1,2$. We will then expand the Kähler form as

$$
\omega=\tau \cdot \tilde{E}_{0}+m \cdot D_{f}+\sum_{i=1}^{2}\left(t_{i}-\frac{\tilde{a}_{i}}{4} \tau\right) \cdot D_{i}^{\prime} .
$$

The index of $Z_{\beta}(\lambda, \tau, m)$ with respect to the geometric elliptic parameter $m$ is

$$
r_{11}^{\beta}=(B+F) \cdot \beta .
$$

Using the Ansatz (4.90) and the genus zero free energy we can immediately fix the numerators

$$
\begin{gathered}
\phi_{F}=\frac{2}{9}\left(\Delta_{4}\right)^{2}\left[-8 A^{2} g+A B\left(4 g^{2}+h\right)+B^{2} g\left(18 g^{2}-5 h\right)\right], \quad \phi_{B}=-2 \sqrt{\Delta_{4}} g \\
\phi_{2 B}=\frac{\Delta_{4}}{288}\left[16 A^{2} g^{2}+8 A B g\left(h-2 g^{2}\right)+B^{2} h\left(3 h-11 g^{2}\right)\right], \\
\phi_{B+F}=\frac{\left(\Delta_{4}\right)^{\frac{5}{2}}}{216}\left[8 A\left(8 C^{2} g^{2}-C D g\left(4 g^{2}+h\right)-D^{2} g^{2}\left(18 g^{2}-5 h\right)\right)\right. \\
B\left(-4 C^{2} g\left(4 g^{2}+33 h\right)+8 C D\left(-4 g^{4}+14 g^{2} h+h^{2}\right)\right. \\
\left.\left.-D^{2} g\left(4 g^{4}-331 g^{2} h+91 h^{2}\right)\right)\right]
\end{gathered}
$$




\begin{tabular}{|c|ccccccc|}
\hline$g \backslash d_{F}$ & 1 & 2 & 3 & 4 & 5 & 6 & 7 \\
\hline 1 & 56 & 0 & 0 & 0 & 0 & 0 & 0 \\
2 & 276 & -4 & 0 & 0 & 0 & 0 & 0 \\
3 & 1360 & -112 & 0 & 0 & 0 & 0 & 0 \\
4 & 4718 & -564 & 6 & 0 & 0 & 0 & 0 \\
5 & 15960 & -3056 & 168 & 0 & 0 & 0 & 0 \\
6 & 46284 & -11108 & 860 & -8 & 0 & 0 & 0 \\
7 & 130064 & -40528 & 4976 & -224 & 0 & 0 & 0 \\
8 & 334950 & -123112 & 18660 & -1164 & 10 & 0 & 0 \\
9 & 837872 & -367552 & 72160 & -7120 & 280 & 0 & 0 \\
10 & 1980756 & -989236 & 226952 & -27392 & 1476 & -12 & 0 \\
11 & 4564224 & -2603520 & 712128 & -111360 & 9488 & -336 & 0 \\
\hline
\end{tabular}

Table 7. GV invariants $n_{d_{F}, d_{E}=0}^{(g)}$ for $\left(F_{4} \rightarrow \mathbb{F}_{1}\right)\left[\mathrm{SU}(2) \times \mathbb{Z}_{2}\right]_{4}^{-144}$ with base class $\beta=B$.

where we have introduced

$$
\begin{aligned}
A & =\phi_{0,1}(2 \tau, \lambda), & B & =\phi_{-2,1}(2 \tau, \lambda), \quad C=\phi_{0,1}(2 \tau, m), \quad D=\phi_{-2,1}(2 \tau, m), \\
g & =E_{2}^{(2)}(\tau), & h & =E_{4}(\tau) .
\end{aligned}
$$

To obtain expressions for other base degrees we have to use additional data. This could, for example, be knowledge about the vanishing of certain Gopakumar-Vafa invariants as was demonstrated in [1].

Some of the Gopakumar-Vafa invariants corresponding to $\beta=B$ and $\beta=B+F$ are listed in the tables 7 and 8 . We label the class of a curve $C$ by the degrees

$$
d_{F}=C \cdot \tilde{E}_{0}, \quad d_{E}=C \cdot D_{f},
$$

and a class $\beta \in H_{2}(B)$. The reflection symmetry along the vertical axis among the invariants 8 with $\beta=B+F$ is a consequence of the invariance under $m \rightarrow-1$. However, there is also a curious periodicity which appears to be present for all genera. This is not at all manifest in the modular expression for $\phi_{B+F}$ (7.69). It should severely constrain the number of free parameters that have to be fixed in the ansatz and it would be very interesting to get a better understand of the origin of this pattern.

\section{Conclusion}

Using homological mirror symmetry we analysed in depth the action of the integral symplectic transformations on the central charges of Type II A and B strings compactified on genus one fibered Calabi-Yau 3-folds $M$ and their mirrors $W$. We considered the case that $M$ had multiple $N$-sections as well as fibral divisors, which respectively lead to Abelian and non-Abelian gauge symmetry enhancements in the Type II - and F-theory vacua. We established that certain auto-equivalences of the category of branes act as $\Gamma_{1}(N)$ on the stringy Kähler moduli space and can be expressed in terms of generic Conifold and 


\begin{tabular}{|c|ccccccccc|}
\hline$d_{F} \backslash d_{E}$ & -4 & -3 & -2 & -1 & 0 & 1 & 2 & 3 & 4 \\
\hline 2 & 0 & 0 & 0 & 0 & 0 & 0 & 0 & 0 & 0 \\
3 & 0 & 0 & 0 & -412 & -1056 & -412 & 0 & 0 & 0 \\
4 & 0 & 0 & -1056 & -16432 & -35072 & -16432 & -1056 & 0 & 0 \\
5 & 0 & -412 & -35072 & -72444 & -73408 & -72444 & -35072 & -412 & 0 \\
6 & 0 & -16432 & -73408 & 4727056 & 9905152 & 4727056 & -73408 & -16432 & 0 \\
7 & -1056 & -72444 & 9905152 & 108929428 & 202048512 & 108929428 & 9905152 & -72444 & -1056 \\
8 & -35072 & 4727056 & 202048512 & 1400552368 & 2439058688 & 1400552368 & 202048512 & 4727056 & -35072 \\
\hline
\end{tabular}

Table 8. GV invariants $n_{d_{F}, d_{E}}^{(g=2)}$ for $\left(F_{4} \rightarrow \mathbb{F}_{1}\right)\left[\mathrm{SU}(2) \times \mathbb{Z}_{2}\right]_{4}^{-144}$ with base class $\beta=B+F$.

large volume monodromies. Together with monodromies that generate the Weyl group this restricts in crucial ways the correlation function of the physical theories. In particular it follows that the topological string partition function can be expressed in terms of Weyl invariant meromorphic Jacobi forms under $\Gamma_{1}(N), N=1,2,3,4$ for each base degree.

We got further insights in the properties of these topological amplitudes by considering their behaviour under those geometric transitions that correspond physically to Higgsing the gauge symmetries. We found that this implies very non-trivial identities among the rings of Jacobi-Foms that occur at the different stages of the Higgsing tree, since the effect of the Higgsing is that the Coulomb branch parameters of the gauge symmetries, which are elliptic parameters of the Jacobi-Forms, are identified with the elliptic arguments which becomes an $N$-th multiple of itself as for example in (4.81). This allows us to generalize the Ansatz for the modular bootstrap on elliptic fibrations to genus one fibrations. At least for low base degrees the partition function can then be fixed by additional boundary conditions.

This partition function is geometrically the most detailed information that is available for the BPS spectrum of the effective physical theories that arise in six, five and four dimensions from F-theory, M-theory and Type II compactifications respectively. For example in 6d F-theory compatifications already the BPS invariants that correspond to the rational curves with base degree zero give the multiplicities of the matter multiplets that arise in co-dimension two in the base.

In the future it would be very interesting to understand the modular properties for genus one fibrations with $N$-sections where $N>4$. Already for $N=5$ the groups $\Gamma_{0}(N)$ and $\Gamma_{1}(N)$ require more than 2 generators and it is not clear how the additional generator manifests as a monodromy in the stringy Kähler moduli space. Also the derivation of the modular properties of the base degree zero partition function cannot easily be generalized. This is particularly exciting because, as we stressed above, the base degree zero part already contains the full information about the spectrum of the corresponding F-theory vacuum. Moreover, it appears that only genus one fibrations with $N \leq 4$ can be realized as complete intersections in toric ambient spaces. If modularity persists for geometries with $N>4$, this should imply an even more intriguing relation involving the monodromies in the stringy Kähler moduli space, the spectra of exotic F-theory vacua, non-toric realizations of genus one fibered Calabi-Yau manifolds and the theory of Jacobi forms. A potential starting point to study this question are the genus one fibrations with 5- and 6-sections that have respectively been constructed in [138] and [139]. 
In the context of heterotic - Type II duality we used the general discussion of the monodromies to argue that heterotic compactifications on CHL orbifolds $\left(K 3 \times T^{2}\right) / \mathbb{Z}_{N}$ should be dual to Type IIA compactifications on genus one fibrations with $N$-sections. We then constructed novel duals of heterotic compactifications on $\left(K 3 \times T^{2}\right) / \mathbb{Z}_{2}$ where $\mathbb{Z}_{2}$ acts as an automorphism of class $2 A$ on the $K 3$ and used the modular bootstrap as well as known results for the corresponding one-loop amplitude on the heterotic side [51] to perform all order checks of the duality. The restriction to $\mathbb{Z}_{2}$, and therefore automorphisms of class $2 A$, is due to the fact that the one-loop amplitudes that can be matched with the topological string partition function has only been calculated for non-standard embeddings on these geometries $[50,51]$. More precisely, the result of the one-loop amplitude can be expressed in terms of the new supersymmetric index and the new supersymmetric index has only been calculated for non-standard embedding on those geometries. Extending the calculation of the new supersymmetric index to compactifications with non-standard embeddings on $\left(K 3 \times T^{2}\right) / \mathbb{Z}_{N}$ with $N>2$ should be relatively straight forward. Matching the results for the corresponding one-loop amplitudes with the topological string partition function on genus one fibrations with 3 - and 4-sections would be a highly non-trivial check of our proposal that we plan to perform in the future.

From the perspective of the $F$-theory effective theory, vacua that arise from $N$-section geometries are obtained by Higgsing $\mathrm{U}(1)$ factors with matter of charge $N$. These are six dimensional, i.e. their effective theory is a chiral six-dimensional supergravity theory with discrete gauge group $\mathbb{Z}_{N}[7,8]$. F-theory duals to the CHL orbifolds of the hererotic string in 8 dimension have been constructed in [140] for $N=2,3,4$. On the type II side they are elliptically fibered $K 3$ with reduced fiber monodromy and reduced rank of the gauge group, which matches the one of the CHL orbifold on $T^{2}$ with an $N$ shift on one of the circles. ${ }^{15}$ A candidate construction for an F-theory/CHL duality in six dimensions could be a fiberwise extension of these eight dimensional CHL orbifolds, similar as the one in the heterotic bundle construction of [142] on elliptic $K 3$. However to maintain the CHL $\mathbb{Z}_{N}$ shift symmetry the elliptically fibered $K 3$ would have to have an $N$-torsional section.

In the recent literature a major amount of effort is dedicated towards a geometric classification of 5d SCFTs [143-152]. In particular, the work [153] discusses a class of 5d SCFTs that is obtained by deformation from KK reductions of 6d SCFTs with twists along the compactification circle (see also [139]). The latter class of theories can be described by M-theory compactifications on local genus one fibered Calabi-Yau threefolds without sections. These rigid theories that come from the $N$-section discussed in section 6 can be also solved by the elliptic blow-up equations similar to the theories discussed in $[18,123$, 124] ${ }^{16}$ In five dimensions, the blow up equations are even more powerful in the sense that the classical topological data is in many cases enough to reconstruct all BPS states [154], i.e. data similar to that which is used to determine the monodromies and anomaly polynomials. It would be very interesting to apply the modular bootstrap for genus one fibrations that we developed in this paper, to study the BPS spectrum of these theories and in particular

\footnotetext{
${ }^{15} \mathrm{~A}$ different construction using a 2-torsional section and half-integral $B$-field on the $\mathbb{P}^{1}$ basis has been proposed in [141].

${ }^{16}$ We thank Xin Wang for pointing this out to us.
} 
to use the 5d blow-up equations and a refined modular bootstrap approach to the ones that involve a twisted circle compactification [153].

\section{A A brief review of the F-theory dictionary}

In this section we review the origin of gauge symmetries in F-theory and the corresponding anomaly coefficients in six dimensions. Most readers will be familiar with the material and can safely skip it. For a thorough review of F-theory we refer to [155] and for a review that focuses on Abelian and discrete gauge symmetries see [156]. We mostly follow the notation of $[85]$.

\section{A.1 Gauge symmetries and matter in F-theory}

Up to a choice of flux and possibly T-brane data the effective physics of a given F-theory vacuum is entirely encoded in the geometry of an elliptically or genus one fibered CalabiYau variety $\pi: X \rightarrow B$. We will only consider smooth threefolds $X$ without multiple or non-flat fibers such that the effective theory is a six-dimensional $(1,0)$-supergravity. Recall that if the theory is further compactified on a circle there is a dual interpretation via M-theory compactified on $X$.

A non-Abelian gauge group $G_{I}$ arises for every irreducible divisor $S_{G_{I}}^{b}:=\left\{\Delta_{I}=0\right\} \subset$ $B$ in the base such that the generic fiber over $S_{G_{I}}^{b}$ is a union of rational curves that intersect like the negative Cartan matrix $-C_{i j}^{G_{I}}$ of the affine Lie algebra associated to $G_{I} \cdot{ }^{17}$ All but one component of the reducible fiber correspond to simple roots $\alpha_{i}, i=1, \ldots, \operatorname{rk}\left(G_{I}\right)$ of $G_{I}$ and will be denoted by $c_{-\alpha_{i}}^{G_{I}}$. The so-called fibral divisors in $X$ that are obtained by fibering $c_{-\alpha_{i}}^{G_{I}}$ over $S_{G_{I}}^{b}$ will be denoted by $D_{i}^{G_{I}}, i=1, \ldots, \operatorname{rk}\left(G_{I}\right)$.

In the M-theory interpretation the 3 -form $C_{3}$ can be expanded along the harmonic forms that correspond to the divisors $D_{i}^{G_{I}}$. This leads to $\operatorname{rk}\left(G_{I}\right)$ massless vector bosons that gauge the Abelian subgroup $\mathrm{U}(1)^{\mathrm{rk}\left(G_{I}\right)} \subset G_{I}$. When the volume of some $c_{-\alpha_{i}}^{G_{I}}$ vanishes, additional massless gauge bosons arise from wrapped M2 branes. In the F-theory limit the volume of the whole fiber is set to zero and one recovers the full unbroken gauge group $G_{I}$.

An elliptically fibered manifold can admit a section that is a rational map $\hat{s}: B \rightarrow X$ such that $\pi \circ \hat{s}=$ id. If $X$ admits at least one section, we can choose a section $\hat{s}_{0}$ and the affine node of the affine Dynkin diagrams associated to the reducible fibers over all $S_{G_{I}}^{b}$ such that $\hat{s}_{0}$ does not intersect any of the $c_{-\alpha_{i}}^{G_{I}}$. Then $\hat{s}_{0}$ is called the zero-section and the name reflects the fact that once a zero-section is chosen the sections form a group with the zero-section as the identity. This group is called the Mordell-Weil group $\mathrm{MW}(X)$ of $X$.

According to the M-theory interpretation the harmonic form that corresponds to the divisor of the zero-section again leads to a massless gauge boson. This can be identified with the Kaluza-Klein gauge boson that arises from reducing the six-dimensional metric along the circle. It disappears in the limit where the circle is decompactified and therefore a single section does not lead to any gauge symmetry in six dimensions.

Additional sections lead to Abelian gauge symmetry if they do not correspond to elements of finite order in the Mordell-Weil group. Given such a non-torsional section $\hat{s}$ we

\footnotetext{
${ }^{17}$ Here we assume that there are no non-simply laced gauge groups.
} 
have to "orthogonalize" it such that M2 branes wrapping the generic fiber or the curves $c_{-\alpha_{i}}^{G_{I}}$ are not charged under the corresponding gauge symmetry. In addition it has to be compatible with the F-theory limit which requires that the dual cohomology class has "one leg along the fiber". Both is achieved by applying the Shioda map $\sigma: \operatorname{MW}(X) \rightarrow \operatorname{NS}(X, \mathbb{Q})$,

$$
\sigma(\hat{s})=S-S_{0}+\pi^{-1}\left(\left[K_{B}\right]-\pi\left(S \cdot S_{0}\right)\right)+\sum_{I}\left(S \cdot c_{-\alpha_{i}}^{G_{I}}\right)\left(C_{G_{I}}^{-1}\right)^{i j} D_{j}^{G_{I}},
$$

where $\operatorname{NS}(X, \mathbb{Q})$ is the Néron-Severi group of divisors modulo numerical equivalence, $S, S_{0}$ are the divisors that correspond to $\hat{s}, \hat{s}_{0}$ respectively and $K_{B}$ is the canonical class of the base. Note that $\mathrm{MW}(X) \rightarrow \mathrm{NS}(X, \mathbb{Q})$ is a group homomorphism and $\mathrm{NS}(X, \mathbb{Q})$ is torsionless. This implies that the image of any torsional section will be trivial. On the other hand, the harmonic form that corresponds to the image of a non-torsional generator of $\mathrm{MW}(X)$ leads to an Abelian gauge group that survives decompactifying the circle.

However, the presence of a torsional section $\hat{s}^{t}$ still has a physical effect. To understand this we first recall the origin of matter in F-theory. Let us consider loci $C$ that are of codimension two in the base where the fiber over $C$ is reducible with $m$ irreducible components. Furthermore let $C$ be contained in $n \geq 0$ divisors $S_{G_{J_{k}}}^{b}, k=1, \ldots, n$ with $J_{a} \neq J_{b}$ for $a \neq b$ and assume that $m>1+\sum_{k=1}^{n} \operatorname{rk}\left(G_{J_{k}}\right)$. The components of the generic fiber over $C$ will intersect like the affine Dynkin diagram of a group $G_{C}$ with

$$
\bigoplus_{k=1}^{n} \mathfrak{g}_{J_{k}} \subset \mathfrak{g}_{C} .
$$

The Dynkin label of the representation with respect to $G_{J_{k}}$ of M2-branes that wrap components of the fiber over $C$ is given by

$$
\lambda_{J_{k}, i}^{c}=D_{i}^{G_{J_{k}}} \cdot c
$$

for $k=1, \ldots, n$. Considering all possible ways in which an M2-brane can wrap components of the fiber (while not wrapping the affine node) this leads to one adjoint representation for every $G_{J_{k}}$ and additional representations that correspond to hypermultiplets from M2branes wrapping e.g. a curve $c$ and some combination of $-c_{-\alpha}^{G_{J_{k}}}$ for $k=1, \ldots, n$ and $\alpha$ roots of the corresponding gauge algebra. The U(1) charges of the latter can be calculated via

$$
q_{j}=\sigma\left(\hat{s}_{j}\right) \cdot c, \quad j=1, \ldots, \operatorname{rk}(M W) .
$$

Now given a torsional section $\hat{s}^{t}$ we already noted that $\sigma\left(\hat{s}^{t}\right)$ is a trivial divisor. This implies that

$$
c \cdot \sum_{I}\left(S^{t} \cdot c_{-\alpha_{i}}^{G_{I}}\right)\left(C_{G_{I}}^{-1}\right)^{i j} D_{j}^{G_{I}}=\sum_{I}\left(S^{t} \cdot c_{-\alpha_{i}}^{G_{I}}\right)\left(C_{G_{I}}^{-1}\right)^{i j} \lambda_{I, j}^{c} \in \mathbb{Z},
$$

for every curve $c$. This is a non-trivial constraint on the Dynkin labels and therefore a torsional section restricts the representations of matter that can occur. In fact, an analogous condition arises also from non-torsional sections. 
Finally we have to discuss what happens when the fibration does not admit a section but only $n$-sections with $n>1$. An $n$-section is locally the union of $n$ sections that are permuted when moving around some divisor in the base. There are various dual pictures to describe this situation but we will only discuss it from the perspective of Higgsing an Abelian gauge group. Consider an elliptic fibration with non-zero Mordell-Weil rank and a generating section $\hat{s}$ as well as isolated fibral curves $C_{ \pm q}$ that intersect with the Shioda map as $\sigma(\hat{s}) \cdot C_{ \pm q}= \pm q$ and therefore lead to hypermultiplets of charge $q$. One can then tune the Kähler moduli such that the volume of one of these curves goes to zero and the corresponding hypermultiplet becomes massless. Turning on a vacuum expectation value for the scalar field amounts to a complex structure deformation that merges $q$ sections and the resulting geometry is a genus one fibration with $q$-sections. The $\mathrm{U}(1)$ factor of the gauge symmetry that corresponded to $\hat{s}$ is broken to $\mathbb{Z}_{q}$. A "Shioda map" for the multisections in the genus one fibration can be obtained by orthogonalization and ensuring the condition that it has "one leg along the fiber".

\section{A.2 The geometric origin of the anomaly polynomial}

In a six-dimensional F-theory vacuum the gauge symmetries itself are anomalous and the anomalies have to be cancelled by a generalized Green-Schwarz mechanism. To this end we note that the six-dimensional spectrum also includes $T=h^{1,1}(B)-1$ tensor multiplets that contain anti-self-dual two-forms $B^{\alpha}, \alpha=2, \ldots, T+1$ and another self-dual two-form $B^{1}$ belongs to the gravity multiplet. Let us introduce a basis $D_{\alpha}, \alpha=1, \ldots, T+1$ of divisors on the base $B$ and write

$$
\Omega_{\alpha \beta}=D_{\alpha} \cdot D_{\beta}
$$

The Green-Schwarz counterterm takes the form

$$
S_{G S}=-\frac{1}{2} \int_{M_{6}} \Omega_{\alpha \beta} B^{\alpha} \wedge X_{4}^{\beta},
$$

with

$$
X_{4}^{\alpha}=\frac{1}{2} a^{\alpha} \operatorname{tr} R \wedge R+2 \sum_{I} \frac{b_{I}^{\alpha}}{\lambda_{I}} \operatorname{tr} F^{I} \wedge F^{I}+2 \sum_{a, b} b_{a b}^{\alpha} F^{a} \wedge F^{b}
$$

Here $R$ is the gravitational field strength, $F^{I}$ is the field strength associated to the factor $G_{I}$ of the non-Abelian gauge group and $F^{a}$ is the Abelian field strength associated to the section $\hat{s}_{a}$. The anomaly coefficients $a^{\alpha}, b_{I}^{\alpha}$ and $b_{a b}^{\alpha}$ are given by

$$
a^{\alpha}=D^{\alpha} \cdot\left[K_{B}\right], \quad b_{I}^{\alpha}=D^{\alpha} \cdot \pi\left(S_{G_{I}}^{b}\right), \quad b_{a b}^{\alpha}=-D^{\alpha} \cdot \pi\left(\sigma\left(\hat{s}_{a}\right) \cdot \sigma\left(\hat{s}_{b}\right)\right),
$$

where $D^{\alpha}=\left(\Omega^{-1}\right)^{\alpha \beta} D_{\beta}$ and $b_{a b}=-\pi\left(\sigma\left(\hat{s}_{a}\right) \cdot \sigma\left(\hat{s}_{b}\right)\right)$ is also called the height pairing. Moreover, $\lambda_{I}$ is a group theoretical normalization constant and for $G_{I}=\mathrm{SU}(N)$ we have $\lambda_{I}=1$.

The anomalies can only be cancelled via the counterterm (A.7) if the one-loop anomaly polynomial $I_{8}$ factorizes as

$$
I_{8}=-\frac{1}{2} \Omega_{\alpha \beta} X_{4}^{\alpha} \wedge X_{4}^{\beta}
$$


This requires non-trivial relations among the multiplicites of representations and the anomaly coefficients. It is striking that the geometrical properties of genus one fibered Calabi-Yau manifold seems to guarantee that these conditions are always satisfied.

\section{B An identity of characteristic classes}

In this appendix we show that

$$
\int_{M} c_{2}(M) D=12 \int_{B} c_{1}(B) \tilde{D}, \quad \text { for } D=\pi^{*} \tilde{D}, \tilde{D} \in H^{1,1}(B),
$$

where $M$ is an elliptically fibered threefold that has been constructed from one of the sixteen toric hypersurfaces that have been discussed in [85]. This implies that the relation holds for a large class of elliptic and genus-one fibrations that exhibit $I_{n}$ fibers, multiple sections and multi-sections. A generic proof of this relation for elliptic fibrations has been given in [84].

Let us denote the rays of the two-dimensional reflexive fiber polytope $\Delta$ by $\rho_{i}, i=$ $1, \ldots, k$ and the corresponding divisors by $D_{i}$ such that they are in clockwise order. The Chern class of the total space of the fibration is given by the restriction of

$$
c(M)=\frac{\left(1+c_{1}(B)\right) \prod_{i=1}^{k}\left(1+D_{i}\right)}{1+c_{1}(B)+\sum_{i=1}^{k} D_{i}},
$$

and therefore the second Chern class $c_{2}(M)$ is the restriction of

$$
c_{2}(M)=c_{1}(B) \sum_{i=1}^{k}+\sum_{i<j}^{k} D_{i} D_{j}
$$

For a vertical divisor $D_{b}=\pi^{*} \tilde{D}$ we can therefore replace

$$
\int_{M} c_{2}(M) D_{b}=\int_{\mathbb{P}} D_{b}\left(c_{1}(B) \sum_{i=1}^{k} D_{i}+\sum_{i<j}^{k} D_{i} D_{j}\right)\left(c_{1}(B)+\sum_{i=1}^{k} D_{i}\right),
$$

where $\mathbb{P}$ is the total ambient space. Further manipulation leads to

$$
\int_{M} c_{2}(M) D_{b}=\underbrace{\int_{\mathbb{P}} D_{b} c_{1}(B)\left[\left(\sum_{i=1}^{k} D_{i}\right)^{2}+\sum_{i<j}^{k} D_{i} D_{j}\right]}_{A}+\underbrace{\int_{\mathbb{P}} D_{b} \sum_{i \neq j}^{k} D_{i}^{2} D_{j}}_{B} .
$$

Since $D_{b} c_{1}(B)$ is the class of a multiple of the generic fiber we can rewrite

$$
\begin{aligned}
A & =\left(\int_{B} \tilde{D} c_{1}(B)\right)\left(\int_{\mathbb{P}_{\Delta}}\left[\left(\sum_{i=1}^{k} D_{i}\right)^{2}+\sum_{i<j}^{k} D_{i} D_{j}\right]\right) \\
& =\left(\int_{B} \tilde{D} c_{1}(B)\right) \int_{\mathbb{P}_{\Delta}}\left(c_{1}(F)^{2}+c_{2}(F)\right),
\end{aligned}
$$


where $\mathbb{P}_{\Delta}$ is the ambient space of the fiber and $c_{i}(F)$ are the corresponding Chern classes. Now the Hirzebruch-Riemann-Roch theorem tells us that

$$
\int_{\mathbb{P}_{\Delta}} \operatorname{Td}(F)=\frac{1}{12} \int_{\mathbb{P}_{\Delta}}\left(c_{1}(F)^{2}+c_{2}(F)\right)=1,
$$

where the second equality reflects that every toric surface is rational. Therefore

$$
A=12 \int_{B} c_{1}(B) \tilde{D}
$$

and our remaining task is to show that $B=0$.

Linear equivalences among toric divisors are of the form

$$
\sum_{i=1}^{k}\left\langle m, \rho_{i}\right\rangle D_{i} \sim 0
$$

for any point $m \in \mathbb{Z}^{2}$. If we consider a bundle where the fibers are given by the toric variety, the right hand side is replaced by $D_{m}=\pi^{*} \tilde{D}_{m}$. Note that the map $m \mapsto D_{m}$ has to be linear. Furthermore note that only the divisors that correspond to neighboring rays in the two-dimensional fan have non-zero intersection.

Let us extend our notation such that $D_{k+1}=D_{1}$ and $D_{0}=D_{k}$. Then

$$
B=\int_{\mathbb{P}} D_{b} \sum_{i=1}^{k}\left[D_{i}^{2} D_{i+1}+D_{i}^{2} D_{i-1}\right] .
$$

Let us denote by $m_{i}$ the ray that is dual to the edge that contains $D_{i}$ and $D_{i+1}$, i.e. $\left\langle m_{i}, \rho_{i}\right\rangle=\left\langle m_{i}, \rho_{i+1}\right\rangle=-1$. Then we can use the linear equivalence corresponding to $m_{i}$ and replace $D_{i}^{2} D_{i+1}$ with $-D_{i} D_{i+1}^{2}+D_{i} D_{i+1} D_{m_{i}}$. But this implies

$$
\begin{aligned}
B & =\int_{\mathbb{P}} D_{b} \sum_{i=1}^{k} D_{i} D_{i+1} D_{m_{i}}=\int_{B} \tilde{D} \sum_{i=1}^{k}\left(\int_{\mathbb{P}_{\Delta}} D_{i} D_{i+1}\right) \tilde{D}_{m_{i}} \\
& =\int_{B} \tilde{D} \sum_{i=1}^{k} \tilde{D}_{m_{i}} .
\end{aligned}
$$

By linearity of the map $m \mapsto \tilde{D}_{m}$ we only have to show that

$$
\sum_{i=1}^{k} m_{i}=0
$$

One can easily verify this for the sixteen reflexive two-dimensional polytopes but let us give an easy argument why this has to be true. As was explained in [157] the intersection of a toric divisor that corresponds to the vertex of the polytope with the generic anti-canonical hypersurface is equal to the number of points on the dual edge minus one. Therefore (B.12) is nothing but the sum over the vertices of $\Delta^{\circ}$ where each vertex is weighted with the 
number of intersections of the corresponding divisor with that curve. Note that the divisors on $\mathbb{P}_{\Delta^{\circ}}$ that correspond to points in the interior of facets do not intersect that curve. Therefore

$$
\sum_{i=1}^{k} m_{i}=\sum_{i=1}^{k^{\prime}}\left(D_{i}^{\prime} \cdot C\right) \rho_{i}^{\prime},
$$

where $\rho_{i}^{\prime}$ are the points on the boundary of $\Delta^{\circ}, D_{i}^{\prime}$ are the corresponding divisors and $C$ is the class of the anti-canonical hypersurface. But the intersections of toric divisors with a curve correspond to the coefficients in a linear equivalence among the corresponding rays. ${ }^{18}$ Therefore the sum has to vanish and (B.1) follows.

\section{Discriminants and Picard-Fuchs systems}

\section{C.1 $\left(F_{6} \rightarrow \mathbb{P}_{2}\right)[\mathrm{U}(1)]_{3}^{-216}$}

\section{Picard-Fuchs operators}

$$
\begin{aligned}
\mathcal{D}_{1}= & \Theta_{1}^{2}+z_{1}\left(\Theta_{1}+1\right)\left(\Theta_{1}-\Theta_{2}+3 \Theta_{3}\right) \\
& -2 z_{1} z_{2}\left(2 \Theta_{1}+\Theta_{2}+3 \Theta_{3}+1\right)\left(3 \Theta_{1}+\Theta_{2}+3 \Theta_{3}+3\right)-2 z_{2}\left(2 \Theta_{1}-\Theta_{2}+3 \Theta_{3}\right) \Theta_{1}, \\
\mathcal{D}_{2}= & \Theta_{1}\left(\Theta_{2}-3 \Theta_{3}\right)-z_{1}\left(\Theta_{1}-\Theta_{2}+3 \Theta_{3}\right)\left(\Theta_{2}+3 \Theta_{3}\right) \\
& -2 z_{1} z_{2}\left(2 \Theta_{1}+\Theta_{2}+3 \Theta_{3}+1\right)\left(4 \Theta_{1}+\Theta_{2}+3 \Theta_{3}+3\right)-4 z_{2} \Theta_{1}\left(2 \Theta_{1}-\Theta_{2}+3 \Theta_{3}\right), \\
\mathcal{D}_{3}= & \Theta_{2}\left(\Theta_{1}-\Theta_{2}+3 z_{2} \Theta_{3}\right)+\left(2 \Theta_{1}-\Theta_{2}+3 \Theta_{3}\right)\left(2 \Theta_{1}+\Theta_{2}+3 \Theta_{3}+1\right), \\
\mathcal{D}_{4}= & \Theta_{1}\left(2 \Theta_{1}-\Theta_{2}+3 \Theta_{3}-1\right)\left(2 \Theta_{1}-\Theta_{2}+3 \Theta_{3}\right) \\
& +z_{1}\left(\Theta_{1}-\Theta_{2}+3 \Theta_{3}\right)\left(2 \Theta_{1}+\Theta_{2}+3 \Theta_{3}+1\right)\left(2 \Theta_{1}+\Theta_{2}+3 \Theta_{3}+2\right), \\
\mathcal{D}_{5}= & -\Theta_{1} \Theta_{2}\left(2 \Theta_{1}-\Theta_{2}+3 \Theta_{3}\right) \\
& +z_{1} z_{2}\left(2 \Theta_{1}+\Theta_{2}+3 \Theta_{3}+1\right)\left(2 \Theta_{1}+\Theta_{2}+3 \Theta_{3}+2\right)\left(2 \Theta_{1}+\Theta_{2}+3 \Theta_{3}+3\right) .
\end{aligned}
$$

\section{Discriminant}

$$
\begin{aligned}
\Delta= & +3 z_{1}+3 z_{1}^{2}+z_{1}^{3}+3 z_{2}-84 z_{1} z_{2}-258 z_{1}^{2} z_{2}-252 z_{1}^{3} z_{2}-81 z_{1}^{4} z_{2} \\
& +3 z_{2}^{2}-465 z_{1} z_{2}^{2}+1782 z_{1}^{2} z_{2}^{2}+7110 z_{1}^{3} z_{2}^{2}+7047 z_{1}^{4} z_{2}^{2}+2187 z_{1}^{5} z_{2}^{2} \\
& +z_{2}^{3}-858 z_{1} z_{2}^{3}+18939 z_{1}^{2} z_{2}^{3}+10500 z_{1}^{3} z_{2}^{3}-55161 z_{1}^{4} z_{2}^{3}-65610 z_{1}^{5} z_{2}^{3} \\
& -19683 z_{1}^{6} z_{2}^{3}-672 z_{1} z_{2}^{4}+56064 z_{1}^{2} z_{2}^{4}-194112 z_{1}^{3} z_{2}^{4}-456192 z_{1}^{4} z_{2}^{4} \\
& -209952 z_{1}^{5} z_{2}^{4}-192 z_{1} z_{2}^{5}+76032 z_{1}^{2} z_{2}^{5}-955008 z_{1}^{3} z_{2}^{5}-1057536 z_{1}^{4} z_{2}^{5} \\
& -139968 z_{1}^{5} z_{2}^{5}+49152 z_{1}^{2} z_{2}^{6}-1978368 z_{1}^{3} z_{2}^{6}-995328 z_{1}^{4} z_{2}^{6}+12288 z_{1}^{2} z_{2}^{7} \\
& -2138112 z_{1}^{3} z_{2}^{7}-331776 z_{1}^{4} z_{2}^{7}-1179648 z_{1}^{3} z_{2}^{8}-262144 z_{1}^{3} z_{2}^{9} \\
& +27 z_{3}-2268 z_{1} z_{2} z_{3}-5832 z_{1} z_{2}^{2} z_{3}+65448 z_{1}^{2} z_{2}^{2} z_{3}-3456 z_{2}^{3} z_{3} \\
& -9072 z_{1} z_{2}^{3} z_{3}+307152 z_{1}^{2} z_{2}^{3} z_{3}-699840 z_{1}^{3} z_{2}^{3} z_{3}-10368 z_{2}^{4} z_{3} \\
& -355104 z_{1} z_{2}^{4} z_{3}-657072 z_{1}^{2} z_{2}^{4} z_{3}-5458752 z_{1}^{3} z_{2}^{4} z_{3}+944784 z_{1}^{4} z_{2}^{4} z_{3} \\
& -10368 z_{2}^{5} z_{3}-1684800 z_{1} z_{2}^{5} z_{3}-2592000 z_{1}^{2} z_{2}^{5} z_{3}-12177216 z_{1}^{3} z_{2}^{5} z_{3} \\
& -3456 z_{2}^{6} z_{3}-2996352 z_{1} z_{2}^{6} z_{3}-2954880 z_{1}^{2} z_{2}^{6} z_{3}-8118144 z_{1}^{3} z_{2}^{6} z_{3} \\
& -2322432 z_{1} z_{2}^{7} z_{3}-1990656 z_{1}^{2} z_{2}^{7} z_{3}-663552 z_{1} z_{2}^{8} z_{3}-663552 z_{1}^{2} z_{2}^{8} z_{3} \\
& -93312 z_{2}^{3} z_{3}^{2}-9517824 z_{1} z_{2}^{4} z_{3}^{2}-16796160 z_{1} z_{2}^{5} z_{3}^{2}-15116544 z_{1}^{2} z_{2}^{5} z_{3}^{2} \\
& +2985984 z_{2}^{6} z_{3}^{2}+6718464 z_{1} z_{2}^{6} z_{3}^{2}+8957952 z_{2}^{7} z_{3}^{2}+4478976 z_{1} z_{2}^{7} z_{3}^{2} \\
& +8957952 z_{2}^{8} z_{3}^{2}+2985984 z_{2}^{9} z_{3}^{2}+80621568 z_{2}^{6} z_{3}^{3} .
\end{aligned}
$$

\footnotetext{
${ }^{18} \mathrm{~A}$ nice explanation of this fact can be found in [158].
} 


\section{$\mathrm{C.2}\left(F_{10} \rightarrow \mathbb{P}_{2}\right)[\mathrm{SU}(2)]_{3}^{-216}$}

\section{Picard-Fuchs operators}

$$
\begin{aligned}
& \mathcal{D}_{1}=-\Theta_{2}\left(-\Theta_{1}+\Theta_{2}-3 \Theta_{3}\right)+z_{2}\left(3 \Theta_{1}-2 \Theta_{2}+6 \Theta_{3}-1\right)\left(3 \Theta_{1}-2 \Theta_{2}+6 \Theta_{3}\right), \\
& \mathcal{D}_{2}=\Theta_{1}\left(3 \Theta_{1}-2 \Theta_{2}+6 \Theta_{3}\right)+z_{1}\left(\Theta_{1}-\Theta_{2}+3 \Theta_{3}\right)\left(3 \Theta_{1}+2 \Theta_{2}+6 \Theta_{3}+3\right) \\
& -2 z_{1} z_{2}\left(9 \Theta_{1}^{2}+6 \Theta_{2} \Theta_{1}+36 \Theta_{3} \Theta_{1}+24 \Theta_{1}-4 \Theta_{2}^{2}+36 \Theta_{3}^{2}-8 \Theta_{2}\right. \\
& \left.+12 \Theta_{2} \Theta_{3}+48 \Theta_{3}+3\right) \text {, } \\
& \mathcal{D}_{3}=2 \Theta_{1}^{2}-\left(\Theta_{1}-\Theta_{2}+3 \Theta_{3}\right)\left(3 \Theta_{1}+2 \Theta_{2}+6 \Theta_{3}+3\right) z_{1}^{2} \\
& +2 z_{2}\left(9 \Theta_{1}^{2}+6 \Theta_{2} \Theta_{1}+36 \Theta_{3} \Theta_{1}+24 \Theta_{1}-4 \Theta_{2}^{2}\right. \\
& \left.+36 \Theta_{3}^{2}-8 \Theta_{2}+12 \Theta_{2} \Theta_{3}+48 \Theta_{3}+3\right) z_{1}^{2} \\
& -16 z_{2}^{2}\left(2 \Theta_{2}+3\right)\left(3 \Theta_{1}-2 \Theta_{2}+6 \Theta_{3}\right) z_{1}+\left(-\Theta_{1}^{2}+2 \Theta_{1}-2 \Theta_{2}+6 \Theta_{3}\right) z_{1} \\
& -4 z_{2}\left(9 \Theta_{1}^{2}+18 \Theta_{3} \Theta_{1}+12 \Theta_{1}+4 \Theta_{2}^{2}+2 \Theta_{2}+18 \Theta_{3}+3\right) z_{1}, \\
& \mathcal{D}_{4}=-\Theta_{1} \Theta_{2}\left(3 \Theta_{1}-2 \Theta_{2}+6 \Theta_{3}\right) \\
& +3 z_{1} z_{2}\left(\Theta_{1}+2 \Theta_{3}+1\right)\left(3 \Theta_{1}+6 \Theta_{3}+1\right)\left(3 \Theta_{1}+6 \Theta_{3}+2\right), \\
& \mathcal{D}_{5}=8 \Theta_{3}^{3}+738 \Theta_{3}^{3} z_{1}^{4}+3072 z_{2} \Theta_{3}^{3} z_{1}{ }^{3}-256 \Theta_{3}^{3} z_{1}{ }^{3}+1728 z_{2}{ }^{2} \Theta_{3}^{3} z_{1}{ }^{2}-1176 z_{2} \Theta_{3}^{3} z_{1}{ }^{2} \\
& +3 z_{3}\left(\Theta_{1}-\Theta_{2}+3 \Theta_{3}\right)\left(381 \Theta_{1}^{2}+80 \Theta_{2} \Theta_{1}+288 \Theta_{3} \Theta_{1}-48 \Theta_{1}\right. \\
& \left.+16 \Theta_{2}^{2}+144 \Theta_{3}^{2}+48 \Theta_{2}+48 \Theta_{2} \Theta_{3}+144 \Theta_{3}+63\right) z_{1}^{2} \\
& -6 z_{2} z_{3}\left(531 \Theta_{1}^{3}+138 \Theta_{2} \Theta_{1}^{2}+2178 \Theta_{3} \Theta_{1}^{2}+1317 \Theta_{1}^{2}-64 \Theta_{2}^{2} \Theta_{1}\right. \\
& +2988 \Theta_{3}^{2} \Theta_{1}-68 \Theta_{2} \Theta_{1}+384 \Theta_{2} \Theta_{3} \Theta_{1}+3336 \Theta_{3} \Theta_{1}+771 \Theta_{1}-32 \Theta_{2}^{3}+1944 \Theta_{3}^{3} \\
& \left.-144 \Theta_{2}^{2}+1512 \Theta_{3}^{2}-190 \Theta_{2}+96 \Theta_{2}^{2} \Theta_{3}+480 \Theta_{2} \Theta_{3}+1290 \Theta_{3}+81\right) z_{1}{ }^{2} \\
& +z_{3}\left(141 \Theta_{1}^{3}+12 \Theta_{2}^{2} \Theta_{1}-144 \Theta_{2} \Theta_{1}-32 \Theta_{1}-648 \Theta_{3}^{3}+72 \Theta_{2}^{2}-648 \Theta_{3}^{2}\right. \\
& \left.+32 \Theta_{2}-96 \Theta_{3}\right) z_{1}+8 z_{2} z_{3}\left(537 \Theta_{1}^{3}-408 \Theta_{2} \Theta_{1}^{2}+2241 \Theta_{3} \Theta_{1}^{2}-180 \Theta_{1}^{2}\right. \\
& -94 \Theta_{2}^{2} \Theta_{1}+2538 \Theta_{3}^{2} \Theta_{1}+223 \Theta_{2} \Theta_{1}+57 \Theta_{1}-96 \Theta_{2}^{3}+1944 \Theta_{3}^{3}-238 \Theta_{2}^{2} \\
& \left.+1350 \Theta_{3}^{2}-191 \Theta_{2}+279 \Theta_{3}-30\right) z_{1}+8 z_{2}^{2} z_{3}\left(351 \Theta_{1}^{3}-96 \Theta_{2} \Theta_{1}^{2}+3834 \Theta_{3} \Theta_{1}^{2}\right. \\
& +738 \Theta_{1}^{2}-488 \Theta_{2}^{2} \Theta_{1}+11124 \Theta_{3}^{2} \Theta_{1}-1776 \Theta_{2} \Theta_{1}-408 \Theta_{2} \Theta_{3} \Theta_{1}+5796 \Theta_{3} \Theta_{1} \\
& -837 \Theta_{1}+384 \Theta_{2}^{3}+9720 \Theta_{3}^{3}+1384 \Theta_{2}^{2}-432 \Theta_{2} \Theta_{3}^{2}+8640 \Theta_{3}^{2}+1224 \Theta_{2} \\
& \left.-1152 \Theta_{2}^{2} \Theta_{3}-4296 \Theta_{2} \Theta_{3}-1242 \Theta_{3}+216\right) z_{1} \\
& +2592 z_{2}^{2} z_{3}^{2} \Theta_{1}\left(2 \Theta_{2}+3\right)\left(3 \Theta_{1}-2 \Theta_{2}+6 \Theta_{3}\right) \\
& -1296 z_{2} z_{3}{ }^{2} \Theta_{1}\left(\Theta_{1}-\Theta_{2}+3 \Theta_{3}\right)\left(5 \Theta_{1}+2 \Theta_{2}+6 \Theta_{3}\right) \\
& +64 z_{2}^{3} z_{3}\left(1296 \Theta_{1}^{3}-1800 \Theta_{2} \Theta_{1}^{2}+5076 \Theta_{3} \Theta_{1}^{2}-585 \Theta_{1}^{2}+600 \Theta_{2}^{2} \Theta_{1}+4752 \Theta_{3}^{2} \Theta_{1}\right. \\
& +228 \Theta_{2} \Theta_{1}-3600 \Theta_{2} \Theta_{3} \Theta_{1}-1440 \Theta_{3} \Theta_{1}-219 \Theta_{1}+8 \Theta_{2}^{3}-432 \Theta_{3}^{3}+72 \Theta_{2}^{2} \\
& \left.-540 \Theta_{3}^{2}+100 \Theta_{2}-144 \Theta_{2} \Theta_{3}-438 \Theta_{3}-15\right) \\
& +24 z_{2} z_{3}\left(11 \Theta_{1}^{3}-12 \Theta_{2}^{2} \Theta_{1}-25 \Theta_{2} \Theta_{1}-16 \Theta_{1}+4 \Theta_{2}^{3}-108 \Theta_{3}^{3}+12 \Theta_{2}^{2}-108 \Theta_{3}^{2}\right. \\
& \left.+14 \Theta_{2}-42 \Theta_{3}\right)+8 z_{3}\left(-\Theta_{2}^{3}+3 \Theta_{1} \Theta_{2}^{2}-3 \Theta_{2}^{2}+6 \Theta_{1} \Theta_{2}-2 \Theta_{2}+27 \Theta_{3}^{3}+27 \Theta_{3}^{2}\right. \\
& \left.+2 \Theta_{1}+6 \Theta_{3}\right)+48 z_{2}^{2} z_{3}\left(261 \Theta_{1}^{3}-136 \Theta_{2} \Theta_{1}^{2}+1062 \Theta_{3} \Theta_{1}^{2}-72 \Theta_{1}^{2}-100 \Theta_{2}^{2} \Theta_{1}\right. \\
& \left.+1116 \Theta_{3}^{2} \Theta_{1}+86 \Theta_{2} \Theta_{1}+57 \Theta_{1}-8 \Theta_{2}^{3}+216 \Theta_{3}^{3}-40 \Theta_{2}^{2}+360 \Theta_{3}^{2}-48 \Theta_{2}+144 \Theta_{3}\right) \text {. }
\end{aligned}
$$




\section{Discriminant components}

$$
\begin{array}{rl}
\Delta_{1}= & +3 z_{1}+3 z_{1}^{2}+z_{1}^{3}-108 z_{1} z_{2}-297 z_{1}^{2} z_{2}-270 z_{1}^{3} z_{2}-81 z_{1}^{4} z_{2} \\
& +4536 z_{1}^{2} z_{2}^{2}+11016 z_{1}^{3} z_{2}^{2}+8667 z_{1}^{4} z_{2}^{2}+2187 z_{1}^{5} z_{2}^{2}-1296 z_{1}^{2} z_{2}^{3} \\
& -95904 z_{1}^{3} z_{2}^{3}-187920 z_{1}^{4} z_{2}^{3}-113724 z_{1}^{5} z_{2}^{3}-19683 z_{1}^{6} z_{2}^{3} \\
& +93312 z_{1}^{3} z_{2}^{4}+1143072 z_{1}^{4} z_{2}^{4}+1469664 z_{1}^{5} z_{2}^{4}+472392 z_{1}^{6} z_{2}^{4} \\
& -2239488 z_{1}^{4} z_{2}^{5}-8118144 z_{1}^{5} z_{2}^{5}-4723920 z_{1}^{6} z_{2}^{5}+559872 z_{1}^{4} z_{2}^{6} \\
& +20715264 z_{1}^{5} z_{2}^{6}+25194240 z_{1}^{6} z_{2}^{6}-20155392 z_{1}^{5} z_{2}^{7}-75582720 z_{1}^{6} z_{2}^{7} \\
& +120932352 z_{1}^{6} z_{2}^{8}-80621568 z_{1}^{6} z_{2}^{9}+27 z_{3}-324 z_{2} z_{3}-2268 z_{1} z_{2} z_{3} \\
& +1296 z_{2}^{2} z_{3}+25920 z_{1} z_{2}^{2} z_{3}+65448 z_{1}^{2} z_{2}^{2} z_{3}-5184 z_{2}^{3} z_{3}-103680 z_{1} z_{2}^{3} z_{3} \\
& -740016 z_{1}^{2} z_{2}^{3} z_{3}-699840 z_{1}^{3} z_{2}^{3} z_{3}-186624 z_{1} z_{2}^{4} z_{3}+1632960 z_{1}^{2} z_{2}^{4} z_{3} \\
& +7138368 z_{1}^{3} z_{2}^{4} z_{3}+944784 z_{1}^{4} z_{2}^{4} z_{3}+5038848 z_{1}^{2} z_{2}^{5} z_{3} \\
& -18475776 z_{1}^{3} z_{2}^{5} z_{3}-18895680 z_{1}^{4} z_{2}^{5} z_{3}-15676416 z_{1}^{2} z_{2}^{6} z_{3} \\
& -15676416 z_{1}^{3} z_{2}^{6} z_{3}+151165440 z_{1}^{4} z_{2}^{6} z_{3}+80621568 z_{1}^{3} z_{2}^{7} z_{3} \\
& -604661760 z_{1}^{4} z_{2}^{7} z_{3}+1209323520 z_{1}^{4} z_{2}^{8} z_{3}-967458816 z_{1}^{4} z_{2}^{9} z_{3} \\
& -93312 z_{2}^{3} z_{3}^{2}+1119744 z_{2}^{4} z_{3}^{2}-9517824 z_{1} z_{2}^{4} z_{3}^{2}-4478976 z_{2}^{5} z_{3}^{2} \\
& +116453376 z_{1} z_{2}^{5} z_{3}^{2}-15116544 z_{1}^{2} z_{2}^{5} z_{3}^{2}+8957952 z_{2}^{6} z_{3}^{2} \\
& -474771456 z_{1} z_{2}^{6} z_{3}^{2}+241864704 z_{1}^{2} z_{2}^{6} z_{3}^{2}+644972544 z_{1} z_{2}^{7} z_{3}^{2} \\
& -1451188224 z_{1}^{2} z_{2}^{7} z_{3}^{2}+3869835264 z_{1}^{2} z_{2}^{8} z_{3}^{2}-3869835264 z_{1}^{2} z_{2}^{9} z_{3}^{2} \\
& +80621568 z_{2}^{6} z_{3}^{3}-967458816 z_{2}^{7} z_{3}^{3}+3869835264 z_{2}^{8} z_{3}^{3}-5159780352 z_{2}^{9} z_{3}^{3} \\
\Delta_{2} & 1-4 z_{2} .
\end{array}
$$

\section{Toric data for geometries with local limits}

Multi-section geometries:

$$
\begin{aligned}
& \left(F_{4} \rightarrow \mathbb{F}_{1}\right)\left[\mathbb{Z}_{2}\right]_{3}^{-224} \\
& \left(\begin{array}{rrrr|rrr}
1 & 0 & -1 & 0 & 0 & 1 & 0 \\
-1 & 0 & -1 & 0 & 0 & 1 & -1 \\
-1 & 1 & -1 & 0 & 0 & 0 & 1 \\
0 & -1 & -1 & 0 & 0 & 0 & 1 \\
0 & 0 & -1 & -1 & 1 & 0 & 0 \\
0 & 0 & -1 & 1 & 1 & 0 & 0 \\
0 & 0 & 1 & 0 & 2 & 2 & 1 \\
0 & 0 & 0 & 0 & -4 & -4 & -2
\end{array}\right)
\end{aligned}
$$




$$
\left(F_{1} \rightarrow \mathbb{F}_{1}\right)\left[\mathbb{Z}_{3}\right]_{3}^{-144}
$$

$$
\left(\begin{array}{rrrr|rrr}
1 & 0 & 0 & 0 & 0 & 1 & 0 \\
-1 & 0 & 0 & 0 & 0 & 1 & -1 \\
-1 & 1 & 0 & 0 & 0 & 0 & 1 \\
0 & -1 & 0 & 0 & 0 & 0 & 1 \\
0 & 0 & 1 & 0 & 1 & 0 & 0 \\
0 & 0 & 0 & 1 & 1 & 0 & 0 \\
0 & 0 & -1 & -1 & 1 & 0 & 0 \\
0 & 0 & 0 & 0 & -3 & 0 & 0
\end{array}\right)
$$

$\left(\mathbb{P}^{3} \rightarrow \mathbb{F}_{\mathbf{1}}\right)\left[\mathbb{Z}_{\mathbf{4}}\right]_{\mathbf{3}}^{-104}$. The label $\Delta_{i}, i \in\{1,2\}$ indicates to which part of the nef-partition the corresponding point belongs:

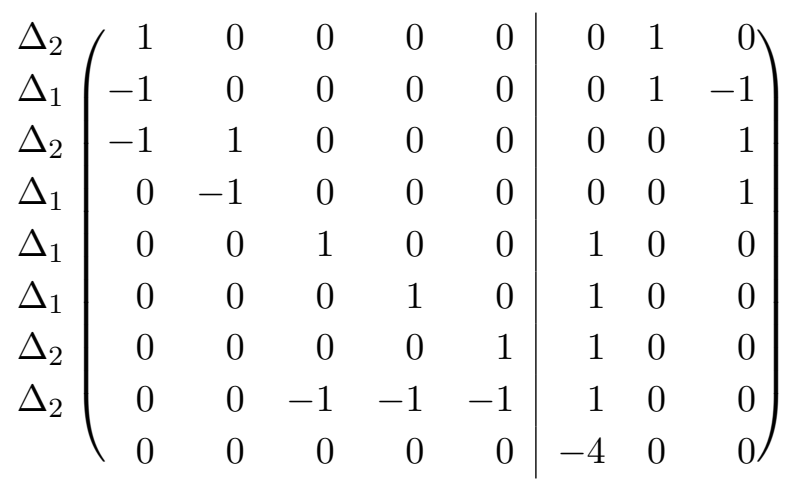

Pseudo multi-section geometries:

$$
\begin{aligned}
& \left(F_{4} \rightarrow \mathbb{F}_{1}\right)\left[\mathbb{Z}_{2}\right]_{4(1)}^{-288} \\
& \left(\begin{array}{rrrr|rrr}
1 & 0 & -1 & -1 & 0 & 1 & 0 \\
-1 & 0 & -1 & -1 & 0 & 1 & -1 \\
-1 & 1 & -1 & -1 & 0 & 0 & 1 \\
0 & -1 & -1 & -1 & 0 & 0 & 1 \\
0 & 0 & -1 & -1 & 1 & -2 & -1 \\
0 & 0 & -1 & 1 & 1 & 0 & 0 \\
0 & 0 & 1 & 0 & 2 & 0 & 0 \\
0 & 0 & 0 & 0 & -4 & 0 & 0
\end{array}\right)
\end{aligned}
$$

$$
\left(F_{1} \rightarrow \mathbb{F}_{1}\right)\left[\mathbb{Z}_{3}\right]_{5(2)}^{-192}
$$

$$
\left(\begin{array}{rrrr|rrr}
1 & 0 & 1 & 0 & 0 & 1 & 0 \\
-1 & 0 & 1 & 0 & 0 & 1 & -1 \\
-1 & 1 & 1 & 0 & 0 & 0 & 1 \\
0 & -1 & 1 & 0 & 0 & 0 & 1 \\
0 & 0 & 1 & 0 & 1 & -2 & -1 \\
0 & 0 & 0 & 1 & 1 & 0 & 0 \\
0 & 0 & -1 & -1 & 1 & 0 & 0 \\
0 & 0 & 0 & 0 & -3 & 0 & 0
\end{array}\right)
$$


$\left(\mathbb{P}^{\mathbf{3}} \rightarrow \mathbb{F}_{\mathbf{1}}\right)\left[\mathbb{Z}_{\mathbf{4}}\right]_{\mathbf{6}(\mathbf{3})}^{-\mathbf{1 2 8}}$. The label $\Delta_{i}, i \in\{1,2\}$ indicates to which part of the nef-partition the corresponding point belongs:

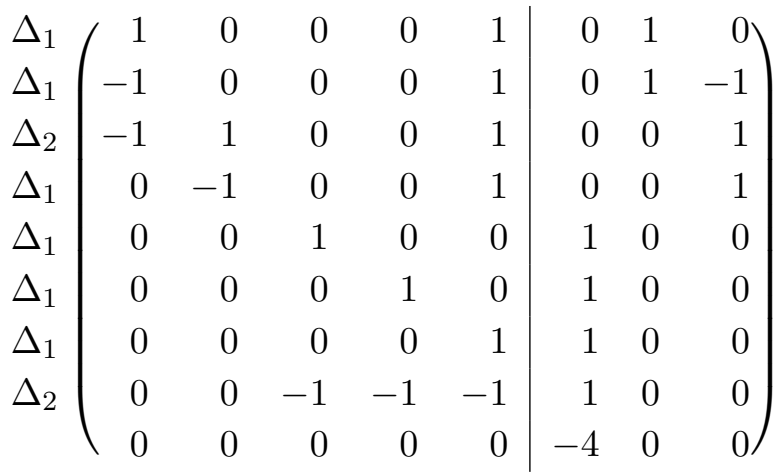

\section{E Modular expressions for refined partition functions}

$$
\begin{aligned}
& \phi_{2}^{(3)}=\frac{\Delta_{6}^{\frac{4}{3}}}{\eta^{24}(3 \tau)}\left(-\frac{145 \mathcal{A}_{+, 3}^{4} \mathcal{A}_{-, 3}^{2} E_{2,3}^{3} E_{6}}{1417176}-\frac{21313 \mathcal{A}_{+, 3}^{4} \mathcal{A}_{-, 3}^{2} E_{2,3}^{2} E_{4}^{2}}{181398528}-\frac{1549 \mathcal{A}_{+, 3}^{4} \mathcal{A}_{-, 3}^{2} E_{2,3} E_{4} E_{6}}{34012224}-\frac{3847 \mathcal{A}_{+, 3}^{4} \mathcal{A}_{-, 3}^{2} E_{4}^{3}}{1088391168}\right. \\
& -\frac{\mathcal{A}_{+, 3}^{4} \mathcal{A}_{-, 3}^{2} E_{6}^{2}}{419904}+\frac{77 \mathcal{A}_{+, 3}^{4} \mathcal{A}_{-, 3} \mathcal{B}_{-, 3} E_{2,3}^{3} E_{4}}{209952}+\frac{2501 \mathcal{A}_{+, 3}^{4} \mathcal{A}_{-, 3} \mathcal{B}_{-, 3} E_{2,3}^{2} E_{6}}{7558272}+\frac{2749 \mathcal{A}_{+, 3}^{4} \mathcal{A}_{-, 3} \mathcal{B}_{-, 3} E_{2,3} E_{4}^{2}}{30233088} \\
& +\frac{\mathcal{A}_{+, 3}^{4} \mathcal{A}_{-, 3} \mathcal{B}_{-, 3} E_{4} E_{6}}{139968}-\frac{295 \mathcal{A}_{+, 3}^{4} \mathcal{B}_{-, 3}^{2} E_{2,3}^{2} E_{4}}{1119744}-\frac{257 \mathcal{A}_{+, 3}^{4} \mathcal{B}_{-, 3}^{2} E_{2,3} E_{6}}{1259712}-\frac{49 \mathcal{A}_{+, 3}^{4} \mathcal{B}_{-, 3}^{2} E_{4}^{2}}{1259712} \\
& +\frac{847 \mathcal{A}_{+, 3}^{3} \mathcal{A}_{-, 3}^{2} \mathcal{B}_{+, 3} E_{2,3}^{3} E_{4}}{839808}+\frac{5089 \mathcal{A}_{+, 3}^{3} \mathcal{A}_{-, 3}^{2} \mathcal{B}_{+, 3} E_{2,3}^{2} E_{6}}{7558272}+\frac{3719 \mathcal{A}_{+, 3}^{3} \mathcal{A}_{-, 3}^{2} \mathcal{B}_{+, 3} E_{2,3} E_{4}^{2}}{30233088}+\frac{\mathcal{A}_{+, 3}^{3} \mathcal{A}_{-, 3}^{2} \mathcal{B}_{+, 3} E_{4} E_{6}}{279936} \\
& -\frac{1555 \mathcal{A}_{+, 3}^{3} \mathcal{A}_{-, 3} \mathcal{B}_{+, 3} \mathcal{B}_{-, 3} E_{2,3}^{2} E_{4}}{559872}-\frac{1157 \mathcal{A}_{+, 3}^{3} \mathcal{A}_{-, 3} \mathcal{B}_{+, 3} \mathcal{B}_{-, 3} E_{2,3} E_{6}}{629856}-\frac{1535 \mathcal{A}_{+, 3}^{3} \mathcal{A}_{-, 3} \mathcal{B}_{+, 3} \mathcal{B}_{-, 3} E_{4}^{2}}{5038848} \\
& +\frac{55 \mathcal{A}_{+, 3}^{3} \mathcal{B}_{+, 3} \mathcal{B}_{-, 3}^{2} E_{2,3}^{3}}{15552}-\frac{25 \mathcal{A}_{+, 3}^{3} \mathcal{B}_{+, 3} \mathcal{B}_{-, 3}^{2} E_{2,3} E_{4}}{31104}-\frac{\mathcal{A}_{+, 3}^{3} \mathcal{B}_{+, 3} \mathcal{B}_{-, 3}^{2} E_{6}}{7776}-\frac{965 \mathcal{A}_{+, 3}^{2} \mathcal{A}_{-, 3}^{2} \mathcal{B}_{+, 3}^{2} E_{2,3}^{2} E_{4}}{373248} \\
& -\frac{643 \mathcal{A}_{+, 3}^{2} \mathcal{A}_{-, 3}^{2} \mathcal{B}_{+, 3}^{2} E_{2,3} E_{6}}{419904}-\frac{751 \mathcal{A}_{+, 3}^{2} \mathcal{A}_{-, 3}^{2} \mathcal{B}_{+, 3}^{2} E_{4}^{2}}{3359232}+\frac{35 \mathcal{A}_{+, 3}^{2} \mathcal{A}_{-, 3} \mathcal{B}_{+, 3}^{2} \mathcal{B}_{-, 3} E_{2,3}^{3}}{2592}+\frac{85 \mathcal{A}_{+, 3} \mathcal{A}_{-, 3}^{2} \mathcal{B}_{+, 3}^{3} E_{2,3}^{3}}{15552} \\
& -\frac{\mathcal{A}_{+, 3}^{2} \mathcal{A}_{-, 3} \mathcal{B}_{+, 3}^{2} \mathcal{B}_{-, 3} E_{6}}{2592}-\frac{13 \mathcal{A}_{+, 3}^{2} \mathcal{B}_{+, 3}^{2} \mathcal{B}_{-, 3}^{2} E_{2,3}^{2}}{3456}+\frac{\mathcal{A}_{+, 3}^{2} \mathcal{B}_{+, 3}^{2} \mathcal{B}_{-, 3}^{2} E_{4}}{3456}-\frac{7 \mathcal{A}_{+, 3}^{2} \mathcal{A}_{-, 3} \mathcal{B}_{+, 3}^{2} \mathcal{B}_{-, 3} E_{2,3} E_{4}}{2592} \\
& -\frac{31 \mathcal{A}_{+, 3} \mathcal{A}_{-, 3}^{2} \mathcal{B}_{+, 3}^{3} E_{2,3} E_{4}}{31104}-\frac{\mathcal{A}_{+, 3} \mathcal{A}_{-, 3}^{2} \mathcal{B}_{+, 3}^{3} E_{6}}{7776}-\frac{47 \mathcal{A}_{+, 3} \mathcal{A}_{-, 3} \mathcal{B}_{+, 3}^{3} \mathcal{B}_{-, 3} E_{2,3}^{2}}{5184}+\frac{5 \mathcal{A}_{+, 3} \mathcal{A}_{-, 3} \mathcal{B}_{+, 3}^{3} \mathcal{B}_{-, 3} E_{4}}{5184} \\
& \left.-\frac{1}{864} \mathcal{A}_{+, 3} \mathcal{B}_{+, 3}^{3} \mathcal{B}_{-, 3}^{2} E_{2,3}-\frac{17 \mathcal{A}_{-, 3}^{2} \mathcal{B}_{+, 3}^{4} E_{2,3}^{2}}{10368}+\frac{\mathcal{A}_{-, 3}^{2} \mathcal{B}_{+, 3}^{4} E_{4}}{5184}+\frac{1}{864} \mathcal{A}_{-, 3} \mathcal{B}_{+, 3}^{4} \mathcal{B}_{-, 3} E_{2,3}+\frac{\mathcal{B}_{+, 3}^{4} \mathcal{B}_{-, 3}^{2}}{288}\right) . \\
& \phi_{2}^{(4)}=\frac{\Delta_{8}^{\frac{1}{2}}}{\eta^{24}(4 \tau)}\left(E_{2,2}-E_{2,4}\right)^{2}\left(\frac{7079 \mathcal{A}_{-, 4}^{2} E_{4}^{3} \mathcal{A}_{+, 4}^{4}}{14495514624}+\frac{179 \mathcal{B}_{-, 4}^{2} E_{4}^{2} \mathcal{A}_{+, 4}^{4}}{63700992}+\frac{3287 \mathcal{A}_{-, 4}^{2} E_{2,4}^{2} E_{4}^{2} \mathcal{A}_{+, 4}^{4}}{1207959552}\right. \\
& -\frac{5263 \mathcal{A}_{-, 4} \mathcal{B}_{-, 4} E_{2,2} E_{4}^{2} \mathcal{A}_{+, 4}^{4}}{11777605632}+\frac{64067 \mathcal{A}_{-, 4} \mathcal{B}_{-, 4} E_{2,4} E_{4}^{2} \mathcal{A}_{+, 4}^{4}}{4416602112}-\frac{1025 \mathcal{A}_{-, 4}^{2} E_{2,2} E_{2,4} E_{4}^{2} \mathcal{A}_{+, 4}^{4}}{37748736} \\
& +\frac{261481 \mathcal{A}_{-, 4}^{2} E_{6}^{2} \mathcal{A}_{+, 4}^{4}}{5087925633024}+\frac{30527 \mathcal{A}_{-, 4} \mathcal{B}_{-, 4} E_{2,4}^{3} E_{4} \mathcal{A}_{+, 4}^{4}}{368050176}+\frac{407 \mathcal{B}_{-, 4}^{2} E_{2,4}^{2} E_{4} \mathcal{A}_{+, 4}^{4}}{5308416}-\frac{1015 \mathcal{B}_{-, 4}^{2} E_{2,2} E_{2,4} E_{4} \mathcal{A}_{+, 4}^{4}}{7077888} \\
& -\frac{61265 \mathcal{A}_{-, 4}^{2} E_{2,4}^{3} E_{6} \mathcal{A}_{+, 4}^{4}}{2944401408}+\frac{28385 \mathcal{A}_{-, 4} \mathcal{B}_{-, 4} E_{2,4}^{2} E_{6} \mathcal{A}_{+, 4}^{4}}{509607936}+\frac{198677 \mathcal{A}_{-, 4} \mathcal{B}_{-, 4} E_{4} E_{6} \mathcal{A}_{+, 4}^{4}}{317995352064} \\
& -\frac{649 \mathcal{B}_{-, 4}^{2} E_{2,2} E_{6} \mathcal{A}_{+, 4}^{4}}{191102976}-\frac{2197 \mathcal{B}_{-, 4}^{2} E_{2,4} E_{6} \mathcal{A}_{+, 4}^{4}}{63700992}+\frac{4837 \mathcal{A}_{-, 4} \mathcal{B}_{-, 4} E_{2,2} E_{2,4} E_{6} \mathcal{A}_{+, 4}^{4}}{1019215872} \\
& -\frac{156265 \mathcal{A}_{-, 4}^{2} E_{2,2} E_{4} E_{6} \mathcal{A}_{+, 4}^{4}}{282662535168}-\frac{1177459 \mathcal{A}_{-, 4}^{2} E_{2,4} E_{4} E_{6} \mathcal{A}_{+, 4}^{4}}{141331267584}+\frac{215 \mathcal{B}_{+, 4} \mathcal{B}_{-, 4}^{2} E_{2,4}^{3} \mathcal{A}_{+, 4}^{3}}{359424} \\
& +\frac{1741 \mathcal{A}_{-, 4} \mathcal{B}_{+, 4} \mathcal{B}_{-, 4} E_{4}^{2} \mathcal{A}_{+, 4}^{3}}{63700992}-\frac{7057 \mathcal{A}_{-, 4}^{2} \mathcal{B}_{+, 4} E_{2,2} E_{4}^{2} \mathcal{A}_{+, 4}^{3}}{11777605632}+\frac{133669 \mathcal{A}_{-, 4}^{2} \mathcal{B}_{+, 4} E_{2,4} E_{4}^{2} \mathcal{A}_{+, 4}^{3}}{4416602112} \\
& +\frac{71113 \mathcal{A}_{-, 4}^{2} \mathcal{B}_{+, 4} E_{2,4}^{3} E_{4} \mathcal{A}_{+, 4}^{3}}{368050176}+\frac{3649 \mathcal{A}_{-, 4} \mathcal{B}_{+, 4} \mathcal{B}_{-, 4} E_{2,4}^{2} E_{4} \mathcal{A}_{+, 4}^{3}}{5308416}+\frac{1693 \mathcal{B}_{+, 4} \mathcal{B}_{-, 4}^{2} E_{2,2} E_{4} \mathcal{A}_{+, 4}^{3}}{103514112}
\end{aligned}
$$




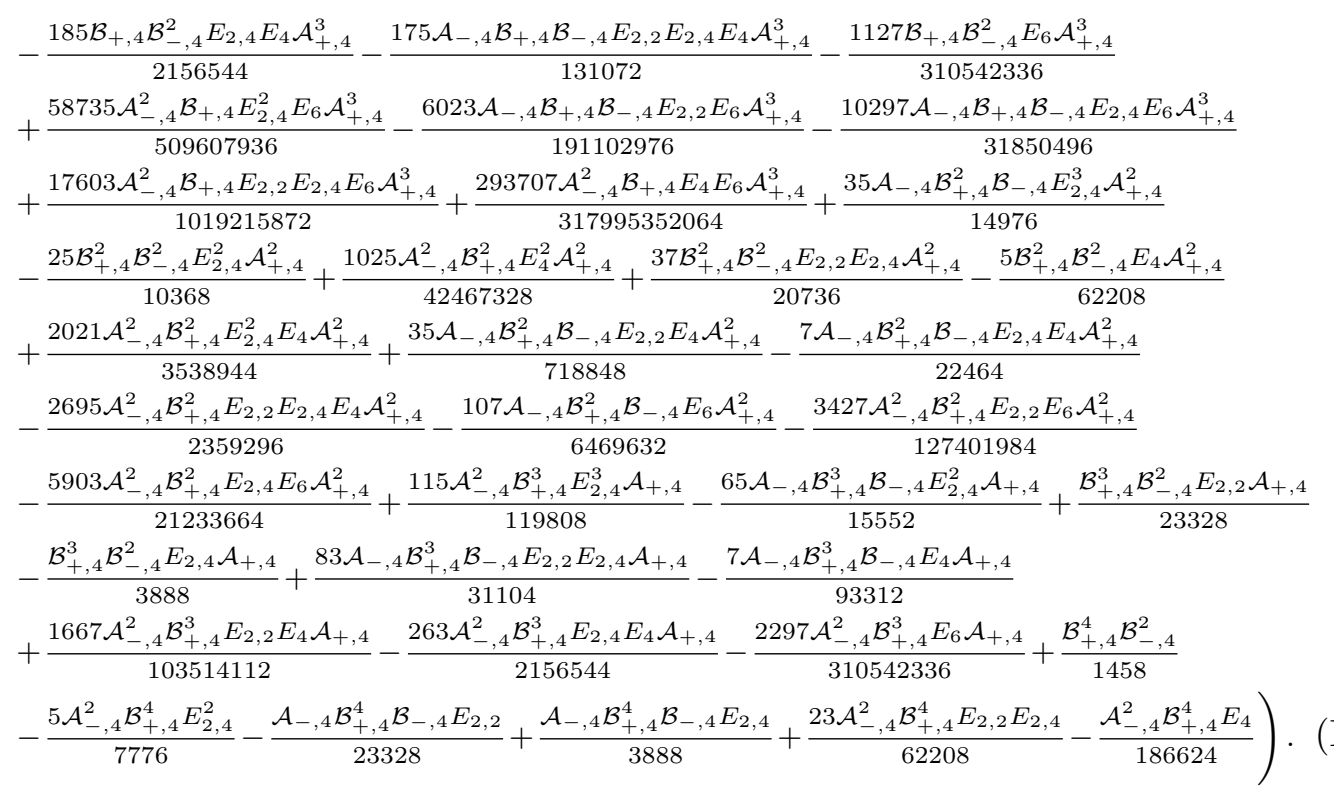

\section{F Refined BPS invariants}

\begin{tabular}{|c|c|c|c|c|c|c|c|c|c|}
\hline$N_{j_{-} j_{+}}^{(1,0)}$ & $2 j_{+}=0$ & $N_{j_{-} j_{+}}^{(1,1)}$ & $2 j_{+}=0$ & $\begin{array}{l}N_{j_{-}}^{(1,2)} j_{+} \\
\text {. }\end{array}$ & $2 j_{+}=0$ & 1 & $\begin{array}{l}N_{j_{-}}^{(1,3)} j_{+}\end{array}$ & $2 j_{+}=0$ & 1 \\
\hline $2 j_{-}=0$ & 16 & $2 j_{-}=0$ & 128 & $2 j_{-}=0$ & 576 & & $2 j_{-}=0$ & 2048 & \\
\hline
\end{tabular}

\begin{tabular}{|c|c|c|c|c|c|c|c|c|c|c|c|c|c|}
\hline \multirow{2}{*}{$N_{j_{-} j_{+}}^{(1,4)}$} & \multirow{2}{*}{$2 j_{+}=0$} & \multirow{2}{*}{1} & \multirow{2}{*}{2} & \multirow{2}{*}{$\begin{array}{l}N_{j_{-} j_{+}}^{(1,5)} \\
\end{array}$} & \multirow{2}{*}{$2 j_{+}=0$} & \multirow{2}{*}{1} & \multirow{2}{*}{2} & \multirow{3}{*}{$\begin{array}{l}N_{j}^{(2,0)} j_{+} \\
\end{array}$} & \multirow{3}{*}{$2 j_{+}=0$} & \multirow{3}{*}{1} & \multirow{3}{*}{$N_{j_{-} j_{+}}^{(2,1)}$} & \multirow{3}{*}{$2 j_{+}=0$} & \multirow{3}{*}{1} \\
\hline & & & & & & & & & & & & & \\
\hline $2 j_{-}=0$ & 6320 & \multirow{3}{*}{592} & & $2 j_{-}=0$ & 17536 & \multirow{3}{*}{2176} & & & & & & & \\
\hline 1 & & & & 1 & & & \multirow[b]{2}{*}{128} & $2 j_{-}=0$ & & 1 & $2 j_{-}=0$ & & 128 \\
\hline 2 & & & 16 & 2 & & & & & & & & & \\
\hline
\end{tabular}

\begin{tabular}{|c|c|c|c|c|c|c|c|c|c|}
\hline$N_{j_{-} j_{+}}^{(2,2)}$ & $2 j_{+}=0$ & 1 & 2 & 3 & $\begin{array}{l}N_{j}^{(2,3)} \\
j_{+}\end{array}$ & $2 j_{+}=0$ & 1 & 2 & 3 \\
\hline $2 j_{-}=0$ & & 1942 & & & $2 j_{-}=0$ & & 15616 & & \\
\hline 1 & 1 & & 121 & & 1 & 128 & & 2176 & \\
\hline 2 & & & & 1 & 2 & & & & 128 \\
\hline
\end{tabular}

\begin{tabular}{|r|rrrrrr|}
\hline$N_{j_{-} j_{+}}^{(2,4)}$ & $2 j_{+}=0$ & 1 & 2 & 3 & 4 & 5 \\
\hline $2 j_{-}=0$ & & 93163 & & 121 & & \\
1 & 2063 & & 19408 & & 1 & \\
2 & & 122 & & 2199 & & \\
3 & & & 1 & & 121 & \\
4 & & & & & & 1
\end{tabular}

Table 9. Refined BPS invariants $N_{j_{-} j_{+}}^{(b, d)}$ of $M_{2}$, for base degree $b \leq 2$ and fiber degree $d \leq 5$. 


\begin{tabular}{|c|c|c|c|c|c|c|c|c|c|c|c|}
\hline$N_{j_{-j}}^{(1,0)}$ & $2 j_{+}=0$ & $N_{j_{-} j_{+}}^{(1,1)}$ & $2 j_{+}=0$ & $N_{j_{-} j_{+}}^{(1,2)}$ & $2 j_{+}=0$ & $N_{j_{-} j_{+}}^{(1,3)}$ & $2 j_{+}=0$ & 1 & $N_{j_{-} j_{+}}^{(1,4)}$ & $2 j_{+}=0$ & 1 \\
\hline $2 j_{-}=0$ & 9 & $2 j_{-}=0$ & 36 & $2 j_{-}=0$ & 126 & $2 j_{-}=0$ & 324 & 9 & $2 j_{-}=0$ & 801 & 36 \\
\hline
\end{tabular}

\begin{tabular}{|c|c|c|c|c|c|c|c|c|c|c|}
\hline $\begin{array}{c}N_{j-j+}^{(1,5)} \\
\end{array}$ & $2 j_{+}=0$ & 1 & 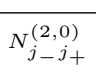 & $2 j_{+}=0$ & 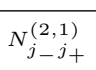 & $2 j_{+}=0$ & 1 & $\begin{array}{c}N_{j-j_{+}}^{(2,2)} \\
.\end{array}$ & $2 j_{+}=0$ & 1 \\
\hline $2 j_{-}=0$ & 1764 & & $2 j_{-}=0$ & & $2 j_{-}=0$ & & 9 & $2 j_{-}=0$ & & 126 \\
\hline
\end{tabular}

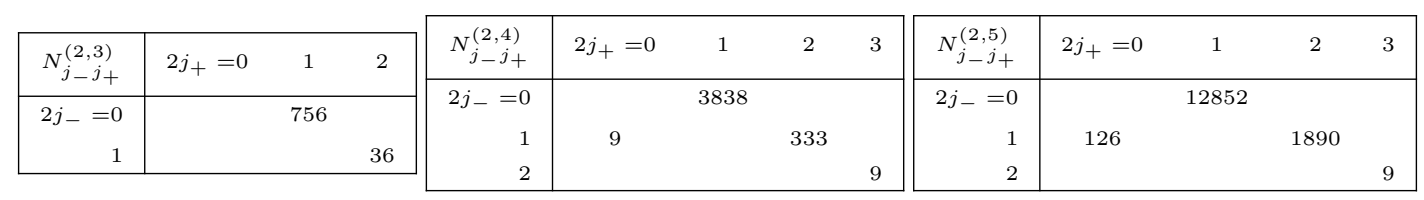

Table 10. Refined BPS invariants $N_{j_{-}, j_{+}}^{(b, d)}$ of $M_{3}$, for base degree $b \leq 2$ and fiber degree $d \leq 5$.

\begin{tabular}{|c|c|c|c|c|c|c|c|c|c|c|}
\hline$N_{j_{-j}}^{(1,0)}$ & $2 j_{+}=0$ & $N_{j_{-j}}^{(1,1)}$ & $2 j_{+}=0$ & $N_{j_{-j} j_{+}}^{(1,2)}$ & $2 j_{+}=0$ & $N_{j_{-j}}^{(1,3)}$ & $2 j_{+}=0$ & $\begin{array}{l}N_{j-j+}^{(1,4)} \\
\end{array}$ & $2 j_{+}=0$ & 1 \\
\hline $2 j_{-}=0$ & 8 & $2 j_{-}=0$ & 16 & $2 j_{-}=0$ & 56 & $2 j_{-}=0$ & 112 & $2 j_{-}=0$ & 248 & \\
\hline
\end{tabular}

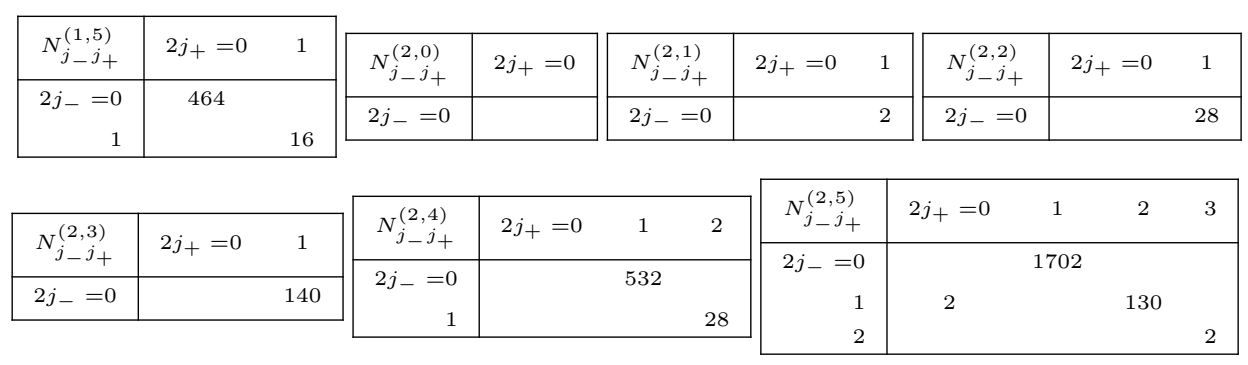

Table 11. Refined BPS invariants $N_{j_{-}, j_{+}}^{(b, d)}$ of $M_{4}$, for base degree $b \leq 2$ and fiber degree $d \leq 5$.

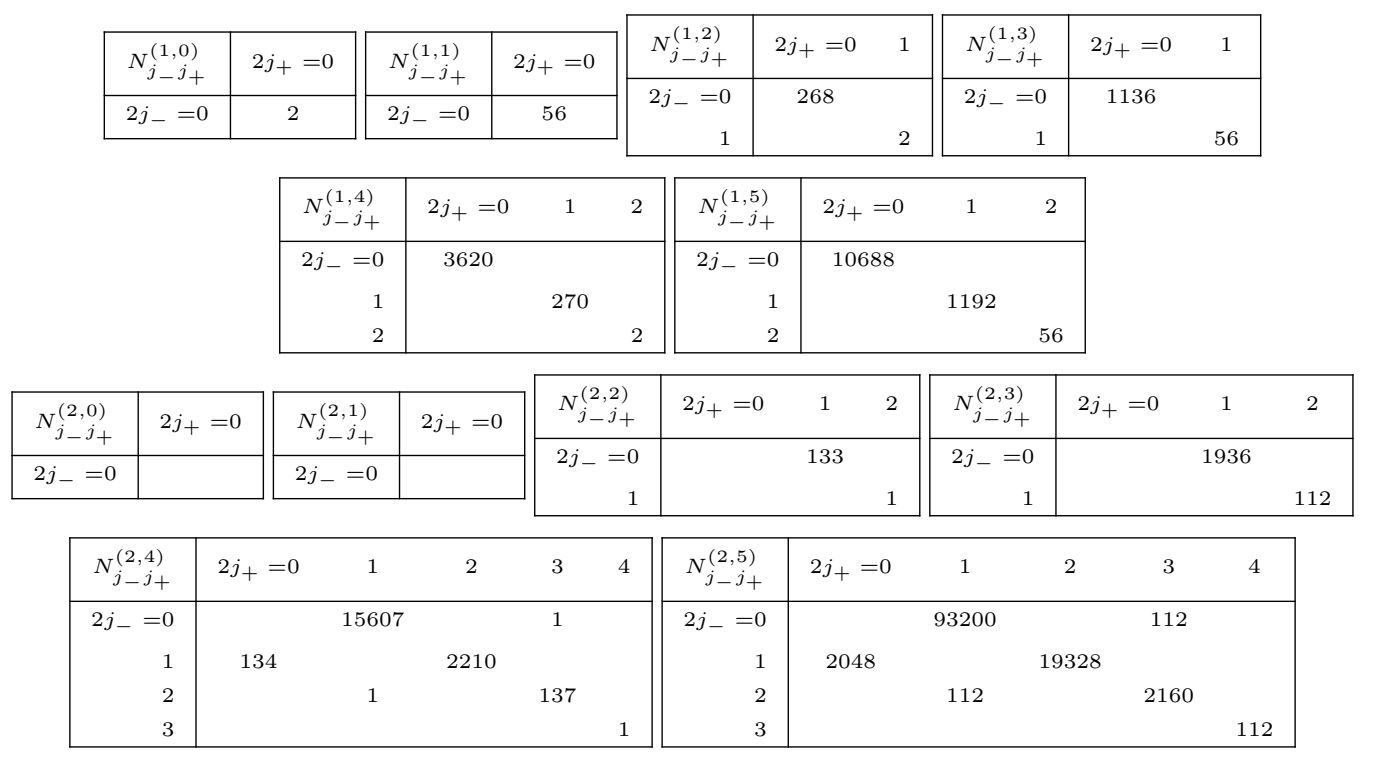

Table 12. Refined BPS invariants $N_{j_{-}, j_{+}}^{(b, d)}$ of $M_{2}^{\prime}$, for base degree $b \leq 2$ and fiber degree $d \leq 5$. 


\begin{tabular}{|c|c|c|c|c|c|c|c|c|c|c|c|}
\hline$N_{j_{-} j_{+}}^{(1,0)}$ & $2 j_{+}=0$ & $N_{j_{-} j_{+}}^{(1,1)}$ & $2 j_{+}=0$ & $N_{j-j_{+}}^{(1,2)}$ & $2 j_{+}=0$ & $\begin{array}{l}N_{j_{-} j_{+}}^{(1,3)} \\
\text {. }\end{array}$ & $2 j_{+}=0$ & 1 & $\begin{array}{l}N_{j_{-}}^{(1,4)} j_{+}\end{array}$ & $2 j_{+}=0$ & 1 \\
\hline $2 j_{-}=0$ & 3 & $2 j_{-}=0$ & 27 & $2 j_{-}=0$ & 81 & $2 j_{-}=0$ & 243 & & $2 j_{-}=0$ & 594 & \\
\hline
\end{tabular}

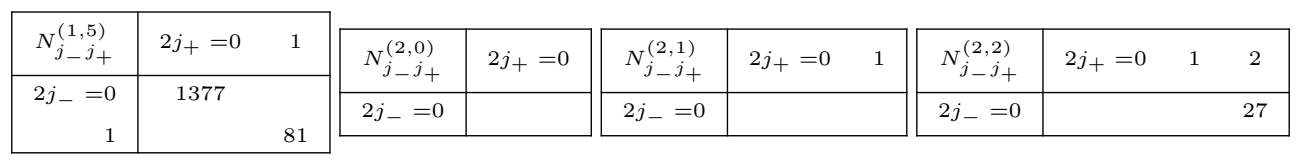

\begin{tabular}{|c|c|c|c|c|c|c|c|c|c|c|c|c|}
\hline $\begin{array}{l}N_{j_{-} j_{+}}^{(2,3)}\end{array}$ & $2 j_{+}=0$ & 1 & 2 & $\begin{array}{l}N_{j_{-} j_{+}}^{(2,4)} \\
\text { (2) }\end{array}$ & $2 j_{+}=0$ & 1 & 2 & $\begin{array}{l}N_{j_{-}}^{(2,5)} j_{+} \\
\text {(n) }\end{array}$ & $2 j_{+}=0$ & 1 & 2 & 3 \\
\hline $\begin{array}{r}2 j_{-}=0 \\
1\end{array}$ & & 237 & 3 & $\begin{array}{r}2 j_{-}=0 \\
1\end{array}$ & 9 & 1296 & 81 & $\begin{array}{r}2 j_{-}=0 \\
1 \\
2\end{array}$ & 27 & 5400 & 621 & 27 \\
\hline
\end{tabular}

Table 13. Refined BPS invariants $N_{j_{-}, j_{+}}^{(b, d)}$ of $M_{3}^{\prime}$, for base degree $b \leq 2$ and fiber degree $d \leq 5$.

\begin{tabular}{|c|c|c|c|c|c|c|c|c|c|c|}
\hline$N_{j_{-} j_{+}}^{(1,0)}$ & $2 j_{+}=0$ \\
\hline $2 j_{-}=0$ & 4 \\
\hline$N_{j_{-} j_{+}}^{(1,1)}$ & $2 j_{+}=0$ & $N_{j_{-} j_{+}}^{(1,2)}$ & $2 j_{+}=0$ \\
\hline $2 j_{-}=0$ & 16 & $2 j_{-}=0$ & 40 & $N_{j_{-} j_{+}}^{(1,3)}$ & $2 j_{+}=0$ \\
\hline $2 j_{-}=0$ & 96 \\
\hline
\end{tabular}

\begin{tabular}{|c|c|c|c|c|c|c|c|c|c|}
\hline $\begin{array}{c}N_{j-j+}^{(1,5)} \\
\end{array}$ & $2 j_{+}=0$ & 1 & $\begin{array}{l}N_{j_{-}}^{(2,0)} j_{+} \\
\text {(n) }\end{array}$ & $2 j_{+}=0$ & $\begin{array}{l}N_{j_{-}}^{(2,1)} j_{+}\end{array}$ & $2 j_{+}=0$ & $N_{j_{-j}}^{(2,2)}$ & $2 j_{+}=0$ & 1 \\
\hline $\begin{array}{r}2 j_{-}=0 \\
1\end{array}$ & 400 & 16 & $2 j_{-}=0$ & & $2 j_{-}=0$ & & $2 j_{-}=0$ & & 10 \\
\hline
\end{tabular}

\begin{tabular}{|c|c|c|c|c|c|c|c|c|c|c|}
\hline$N_{j}^{(2,3)}$ & $2 j_{+}=0$ & 1 & $N_{j_{-} j_{+}}^{(2,4)}$ & $2 j_{+}=0$ & 1 & 2 & $\begin{array}{l}N_{j}^{(2,4)} \\
j_{+}\end{array}$ & $2 j_{+}=0$ & 1 & 2 \\
\hline $2 j_{-}=0$ & & 64 & $\begin{array}{r}2 j_{-}=0 \\
1\end{array}$ & & 286 & 6 & $\begin{array}{r}2 j_{-}=0 \\
1\end{array}$ & & 960 & 64 \\
\hline
\end{tabular}

Table 14. Refined BPS invariants $N_{j_{-}, j_{+}}^{(b, d)}$ of $M_{4}^{\prime}$, for base degree $b \leq 2$ and fiber degree $d \leq 5$.

Open Access. This article is distributed under the terms of the Creative Commons Attribution License (CC-BY 4.0), which permits any use, distribution and reproduction in any medium, provided the original author(s) and source are credited.

\section{References}

[1] M.-x. Huang, S. Katz and A. Klemm, Topological String on elliptic CY 3-folds and the ring of Jacobi forms, JHEP 10 (2015) 125 [arXiv:1501.04891] [INSPIRE].

[2] M.-x. Huang, S. Katz and A. Klemm, Elliptifying topological string theory, in preparation.

[3] J.H. Silverman and J.T. Tate, Rational points on elliptic curves, second edition, Undergraduate Texts in Mathematics, Springer, Cham, (2015),

[https://doi.org/10.1007/978-3-319-18588-0].

[4] P.S. Aspinwall and D.R. Morrison, Nonsimply connected gauge groups and rational points on elliptic curves, JHEP 07 (1998) 012 [hep-th/9805206] [INSPIRE].

[5] D.R. Morrison and C. Vafa, Compactifications of F-theory on Calabi-Yau threefolds. 2., Nucl. Phys. B 476 (1996) 437 [hep-th/9603161] [INSPIRE].

[6] A. Klemm, P. Mayr and C. Vafa, BPS states of exceptional noncritical strings, Nucl. Phys. Proc. Suppl. 58 (1997) 177 [hep-th/9607139] [INSPIRE]. 
[7] J. de Boer et al., Triples, fluxes and strings, Adv. Theor. Math. Phys. 4 (2002) 995 [hep-th/0103170] [INSPIRE].

[8] D.R. Morrison and W. Taylor, Sections, multisections and U(1) fields in F-theory, arXiv: 1404.1527 [INSPIRE].

[9] M. Bershadsky, K.A. Intriligator, S. Kachru, D.R. Morrison, V. Sadov and C. Vafa, Geometric singularities and enhanced gauge symmetries, Nucl. Phys. B 481 (1996) 215 [hep-th/9605200] [INSPIRE].

[10] E. Witten, Quantum background independence in string theory, in Conference on Highlights of Particle and Condensed Matter Physics (SalamFEST) Trieste, Italy, March 8-12, 1993, pp. 0257-275, 1993, hep-th/9306122 [INSPIRE].

[11] A. Klemm, J. Manschot and T. Wotschke, Quantum geometry of elliptic Calabi-Yau manifolds, arXiv:1205.1795 [INSPIRE].

[12] A. Dabholkar, S. Murthy and D. Zagier, Quantum Black Holes, Wall Crossing and Mock Modular Forms, arXiv: 1208.4074 [INSPIRE].

[13] T. Schimannek, Modularity from Monodromy, JHEP 05 (2019) 024 [arXiv:1902.08215] [INSPIRE].

[14] B. Haghighat, A. Iqbal, C. Kozçaz, G. Lockhart and C. Vafa, M-Strings, Commun. Math. Phys. 334 (2015) 779 [arXiv:1305.6322] [INSPIRE].

[15] B. Haghighat, A. Klemm, G. Lockhart and C. Vafa, Strings of Minimal 6d SCFTs, Fortsch. Phys. 63 (2015) 294 [arXiv:1412.3152] [INSPIRE].

[16] B. Haghighat, S. Murthy, C. Vafa and S. Vandoren, F-Theory, Spinning Black Holes and Multi-string Branches, JHEP 01 (2016) 009 [arXiv: 1509.00455] [INSPIRE].

[17] M. Del Zotto and G. Lockhart, On Exceptional Instanton Strings, JHEP 09 (2017) 081 [arXiv: 1609.00310] [INSPIRE].

[18] J. Gu, M.-x. Huang, A.-K. Kashani-Poor and A. Klemm, Refined BPS invariants of $6 d$ SCFTs from anomalies and modularity, JHEP 05 (2017) 130 [arXiv:1701.00764] [INSPIRE].

[19] M. Del Zotto, J. Gu, M.-X. Huang, A.-K. Kashani-Poor, A. Klemm and G. Lockhart, Topological Strings on Singular Elliptic Calabi-Yau 3-folds and Minimal 6d SCFTs, JHEP 03 (2018) 156 [arXiv: 1712.07017] [INSPIRE].

[20] M. Del Zotto and G. Lockhart, Universal Features of BPS Strings in Six-dimensional SCFTs, JHEP 08 (2018) 173 [arXiv: 1804.09694] [INSPIRE].

[21] S.-J. Lee, W. Lerche and T. Weigand, Tensionless Strings and the Weak Gravity Conjecture, JHEP 10 (2018) 164 [arXiv:1808.05958] [INSPIRE].

[22] S.-J. Lee, W. Lerche and T. Weigand, A Stringy Test of the Scalar Weak Gravity Conjecture, Nucl. Phys. B 938 (2019) 321 [arXiv:1810.05169] [INSPIRE].

[23] C.F. Cota, A. Klemm and T. Schimannek, Modular Amplitudes and Flux-Superpotentials on elliptic Calabi-Yau fourfolds, JHEP 01 (2018) 086 [arXiv:1709.02820] [INSPIRE].

[24] M. Aganagic, V. Bouchard and A. Klemm, Topological Strings and (Almost) Modular Forms, Commun. Math. Phys. 277 (2008) 771 [hep-th/0607100] [InSPIRE].

[25] M. Günaydin, A. Neitzke and B. Pioline, Topological wave functions and heat equations, JHEP 12 (2006) 070 [hep-th/0607200] [INSPIRE]. 
[26] T. Bridgeland, Fourier-mukai transforms for elliptic surfaces, alg-geom/9705002.

[27] B. Andreas, G. Curio, D.H. Ruiperez and S.-T. Yau, Fourier-Mukai transform and mirror symmetry for D-branes on elliptic Calabi-Yau, math/0012196.

[28] B. Andreas, G. Curio, D. Hernández Ruipérez and S.-T. Yau, Fiber wise T duality for D-branes on elliptic Calabi-Yau, JHEP 03 (2001) 020 [hep-th/0101129] [INSPIRE].

[29] B. Andreas and D. Hernández Ruipérez, Fourier Mukai transforms and applications to string theory, math/0412328.

[30] D.H. Ruipérez, A. Martín and F.S. de Salas, Relative integral functors for singular fibrations and singular partners, math/0610319.

[31] A. Klemm and P. Mayr, Strong coupling singularities and nonAbelian gauge symmetries in $N=2$ string theory, Nucl. Phys. B 469 (1996) 37 [hep-th/9601014] [INSPIRE].

[32] S.H. Katz, D.R. Morrison and M.R. Plesser, Enhanced gauge symmetry in type-II string theory, Nucl. Phys. B 477 (1996) 105 [hep-th/9601108] [InSPIRE].

[33] R.P. Horja, Derived category automorphisms from mirror symmetry, math/0103231.

[34] B. Szendroi, Enhanced gauge symmetry and braid group actions, Commun. Math. Phys. 238 (2003) 35 [math/0210122] [inSPIRE].

[35] B. Szendroi, Artin group actions on derived categories of threefolds, math/0210121.

[36] P.S. Aspinwall, Some navigation rules for D-brane monodromy, J. Math. Phys. 42 (2001) 5534 [hep-th/0102198] [INSPIRE].

[37] A. Kashani-Poor, Identifying matter in F-theory via the topological string, Talk at CERN, June 2019, work in progress.

[38] P. Oehlmann and T. Schimannek, work in progress.

[39] S. Kachru, A. Klemm and Y. Oz, Calabi-Yau duals for CHL strings, Nucl. Phys. B 521 (1998) 58 [hep-th/9712035] [INSPIRE].

[40] S. Datta, J.R. David and D. Lüst, Heterotic string on the CHL orbifold of K3, JHEP 02 (2016) 056 [arXiv: 1510.05425] [INSPIRE].

[41] S. Chaudhuri, G. Hockney and J.D. Lykken, Maximally supersymmetric string theories in $D<10$, Phys. Rev. Lett. 75 (1995) 2264 [hep-th/9505054] [INSPIRE].

[42] S. Chaudhuri and J. Polchinski, Moduli space of CHL strings, Phys. Rev. D 52 (1995) 7168 [hep-th/9506048] [INSPIRE].

[43] A. Klemm, W. Lerche and P. Mayr, K3 Fibrations and heterotic type-II string duality, Phys. Lett. B 357 (1995) 313 [hep-th/9506112] [INSPIRE].

[44] S. Kachru, A. Klemm, W. Lerche, P. Mayr and C. Vafa, Nonperturbative results on the point particle limit of $N=2$ heterotic string compactifications, Nucl. Phys. B 459 (1996) 537 [hep-th/9508155] [INSPIRE].

[45] J.H. Schwarz and A. Sen, Type IIA dual of the six-dimensional CHL compactification, Phys. Lett. B 357 (1995) 323 [hep-th/9507027] [INSPIRE].

[46] S. Mukai, Finite groups of automorphisms of K3 surfaces and the Mathieu group., Invent. Math. 94 (1988) 183. 
[47] M.R. Gaberdiel, S. Hohenegger and R. Volpato, Symmetries of K3 $\sigma$-models, Commun. Num. Theor. Phys. 6 (2012) 1 [arXiv:1106.4315] [INSPIRE].

[48] M.C.N. Cheng, S.M. Harrison, R. Volpato and M. Zimet, K3 String Theory, Lattices and Moonshine, arXiv: 1612.04404 [INSPIRE].

[49] N.M. Paquette, R. Volpato and M. Zimet, No More Walls! A Tale of Modularity, Symmetry and Wall Crossing for 1/4 BPS Dyons, JHEP 05 (2017) 047 [arXiv: 1702.05095] [INSPIRE].

[50] A. Chattopadhyaya and J.R. David, $\mathcal{N}=2$ heterotic string compactifications on orbifolds of $K 3 \times T^{2}, J H E P 01(2017) 037$ [arXiv:1611.01893] [INSPIRE].

[51] A. Chattopadhyaya and J.R. David, Gravitational couplings in $\mathcal{N}=2$ string compactifications and Mathieu Moonshine, JHEP 05 (2018) 211 [arXiv:1712.08791] [INSPIRE].

[52] I. Antoniadis, E. Gava, K.S. Narain and T.R. Taylor, Topological amplitudes in string theory, Nucl. Phys. B 413 (1994) 162 [hep-th/9307158] [InSPIRE].

[53] I. Antoniadis, E. Gava, K.S. Narain and T.R. Taylor, $N=2$ type-II heterotic duality and higher derivative F terms, Nucl. Phys. B 455 (1995) 109 [hep-th/9507115] [INSPIRE].

[54] M. Mariño and G.W. Moore, Counting higher genus curves in a Calabi-Yau manifold, Nucl. Phys. B 543 (1999) 592 [hep-th/9808131] [INSPIRE].

[55] A. Klemm and M. Mariño, Counting BPS states on the enriques Calabi-Yau, Commun. Math. Phys. 280 (2008) 27 [hep-th/0512227] [INSPIRE].

[56] W. Nahm, Supersymmetries and their Representations, Nucl. Phys. B 135 (1978) 149 [INSPIRE].

[57] J.J. Heckman, D.R. Morrison and C. Vafa, On the Classification of 6D SCFTs and Generalized ADE Orbifolds, JHEP 05 (2014) 028 [Erratum ibid. 06 (2015) 017] [arXiv:1312.5746] [INSPIRE].

[58] J.J. Heckman, D.R. Morrison, T. Rudelius and C. Vafa, Atomic Classification of 6D SCFTs, Fortsch. Phys. 63 (2015) 468 [arXiv:1502.05405] [INSPIRE].

[59] J. Kim, S. Kim, K. Lee, J. Park and C. Vafa, Elliptic Genus of E-strings, JHEP 09 (2017) 098 [arXiv: 1411.2324] [INSPIRE].

[60] V.V. Batyrev, Dual polyhedra and mirror symmetry for calabi-yau hypersurfaces in toric varieties, alg-geom/9310003.

[61] V.V. Batyrev and L.A. Borisov, On Calabi-Yau complete intersections in toric varieties, alg-geom/9412017 [INSPIRE].

[62] S. Hosono, A. Klemm, S. Theisen and S.-T. Yau, Mirror symmetry, mirror map and applications to Calabi-Yau hypersurfaces, Commun. Math. Phys. 167 (1995) 301 [hep-th/9308122] [INSPIRE].

[63] S. Hosono, A. Klemm, S. Theisen and S.-T. Yau, Mirror symmetry, mirror map and applications to complete intersection Calabi-Yau spaces, Nucl. Phys. B 433 (1995) 501 [hep-th/9406055] [INSPIRE].

[64] M. Bershadsky, S. Cecotti, H. Ooguri and C. Vafa, Holomorphic anomalies in topological field theories, Nucl. Phys. B 405 (1993) 279 [hep-th/9302103] [INSPIRE].

[65] R. Wazir, Arithmetic on Elliptic Threefolds, math/0112259. 
[66] V. Braun and D.R. Morrison, F-theory on Genus-One Fibrations, JHEP 08 (2014) 132 [arXiv:1401.7844] [INSPIRE].

[67] D.S. Park, Anomaly Equations and Intersection Theory, JHEP 01 (2012) 093 [arXiv:1111.2351] [INSPIRE].

[68] M. Alim and E. Scheidegger, Topological Strings on Elliptic Fibrations, Commun. Num. Theor. Phys. 08 (2014) 729 [arXiv:1205.1784] [INSPIRE].

[69] E. Witten, Phases of $N=2$ theories in two-dimensions, Nucl. Phys. B 403 (1993) 159 [hep-th/9301042] [INSPIRE].

[70] P.S. Aspinwall, B.R. Greene and D.R. Morrison, Calabi-Yau moduli space, mirror manifolds and space-time topology change in string theory, Nucl. Phys. B 416 (1994) 414 [hep-th/9309097] [INSPIRE].

[71] M. Kontsevich, Homological Algebra of Mirror Symmetry, alg-geom/9411018 [INSPIRE].

[72] P.S. Aspinwall, D-branes on Calabi-Yau manifolds, in Progress in string theory. Proceedings, Summer School, TASI 2003, Boulder, U.S.A., June 2-27, 2003, pp. 1-152, 2004, hep-th/0403166 [INSPIRE].

[73] M.R. Douglas, D-branes, categories and $N=1$ supersymmetry, J. Math. Phys. 42 (2001) 2818 [hep-th/0011017] [INSPIRE].

[74] H. Iritani, An integral structure in quantum cohomology and mirror symmetry for toric orbifolds, arXiv:0903.1463.

[75] A. Gerhardus and H. Jockers, Quantum periods of Calabi-Yau fourfolds, Nucl. Phys. B 913 (2016) 425 [arXiv: 1604.05325] [INSPIRE].

[76] D. Orlov, Equivalences of derived categories and K3 surfaces, alg-geom/9606006.

[77] R.P. Horja, Hypergeometric functions and mirror symmetry in toric varieties, math/9912109.

[78] J. Distler, H. Jockers and H.-j. Park, D-brane monodromies, derived categories and boundary linear $\sigma$-models, hep-th/0206242 [INSPIRE].

[79] P. Seidel and R.P. Thomas, Braid group actions on derived categories of coherent sheaves, math/0001043.

[80] P.S. Aspinwall and M.R. Douglas, D-brane stability and monodromy, JHEP 05 (2002) 031 [hep-th/0110071] [INSPIRE].

[81] D.R. Morrison, Geometric aspects of mirror symmetry, math/0007090.

[82] D. Huybrechts, M. Huybrechts and O.U. Press, Fourier-Mukai Transforms in Algebraic Geometry, Oxford Mathematical Monographs, Clarendon Press, (2006).

[83] W. Fulton, Intersection theory, Ergebnisse der Mathematik und ihrer Grenzgebiete, Springer-Verlag, (1984).

[84] M. Esole and M.J. Kang, Characteristic numbers of elliptic fibrations with non-trivial Mordell-Weil groups, arXiv: 1808.07054 [INSPIRE].

[85] D. Klevers, D.K. Mayorga Pena, P.-K. Oehlmann, H. Piragua and J. Reuter, F-Theory on all Toric Hypersurface Fibrations and its Higgs Branches, JHEP 01 (2015) 142 [arXiv: 1408.4808] [INSPIRE]. 
[86] J.H. Silverman, The Arithmetic of Elliptic Curves, Graduate Texts in Mathematics, Springer, Dordrecht, (2009), [https://doi.org/10.1007/978-0-387-09494-6].

[87] R.P. Horja, Hypergeometric functions and mirror symmetry in toric varieties, math/9912109.

[88] P.S. Aspinwall, R.L. Karp and R.P. Horja, Massless D-branes on Calabi-Yau threefolds and monodromy, Commun. Math. Phys. 259 (2005) 45 [hep-th/0209161] [INSPIRE].

[89] K. Oguiso, On algebraic fiber space structures on a calabi-yau 3-fold, Int. J. Math. 04 (1993) 439.

[90] R. Blumenhagen, D. Kläwer, L. Schlechter and F. Wolf, The Refined Swampland Distance Conjecture in Calabi-Yau Moduli Spaces, JHEP 06 (2018) 052 [arXiv:1803.04989] [INSPIRE].

[91] S.-J. Lee, W. Lerche and T. Weigand, Modular Fluxes, Elliptic Genera and Weak Gravity Conjectures in Four Dimensions, JHEP 08 (2019) 104 [arXiv:1901.08065] [INSPIRE].

[92] D. Erkinger and J. Knapp, Refined swampland distance conjecture and exotic hybrid Calabi-Yaus, JHEP 07 (2019) 029 [arXiv:1905.05225] [INSPIRE].

[93] A. Joshi and A. Klemm, Swampland Distance Conjecture for One-Parameter Calabi-Yau Threefolds, JHEP 08 (2019) 086 [arXiv: 1903.00596] [INSPIRE].

[94] E. Palti, The Swampland: Introduction and Review, Fortsch. Phys. 67 (2019) 1900037 [arXiv: 1903.06239] [INSPIRE].

[95] P.S. Aspinwall and R.L. Karp, Solitons in Seiberg-Witten theory and D branes in the derived category, JHEP 04 (2003) 049 [hep-th/0211121] [INSPIRE].

[96] E. Witten, Phase transitions in M-theory and F-theory, Nucl. Phys. B 471 (1996) 195 [hep-th/9603150] [INSPIRE].

[97] P. Corvilain, T.W. Grimm and I. Valenzuela, The Swampland Distance Conjecture for Kähler moduli, JHEP 08 (2019) 075 [arXiv: 1812.07548] [INSPIRE].

[98] S.-J. Lee, W. Lerche and T. Weigand, Emergent Strings from Infinite Distance Limits, arXiv: 1910.01135 [INSPIRE].

[99] M. Herbst, K. Hori and D. Page, Phases Of $N=2$ Theories In $1+1$ Dimensions With Boundary, arXiv:0803.2045 [INSPIRE].

[100] D. Erkinger and J. Knapp, Hemisphere Partition Function and Monodromy, JHEP 05 (2017) 150 [arXiv: 1704.00901] [INSPIRE].

[101] S. Katz, Elliptically fibered Calabi-Yau threefolds: mirror symmetry and Jacobi forms, July, 2016, https://indico.cern.ch/event/375104/contributions/2153264/.

[102] M. Eichler and D. Zagier, The theory of Jacobi forms, Progress in mathematics, Birkhäuser, (1985).

[103] C. Ziegler, Jacobi forms of higher degree, Abhandlungen aus dem Mathematischen Seminar der Universität Hamburg 59 (1989) 191.

[104] E.P. Verlinde, Attractors and the holomorphic anomaly, hep-th/0412139 [INSPIRE].

[105] S. Kachru and C. Vafa, Exact results for $N=2$ compactifications of heterotic strings, Nucl. Phys. B 450 (1995) 69 [hep-th/9505105] [INSPIRE]. 
[106] P.S. Aspinwall, K3 surfaces and string duality, in Differential geometry inspired by string theory, pp. 421-540, 1996, hep-th/9611137 [INSPIRE].

[107] M. Serone, $N=2$ type-I heterotic duality and higher derivative F terms, Phys. Lett. B 395 (1997) 42 [Erratum ibid. B 401 (1997) 363] [hep-th/9611017] [INSPIRE].

[108] R.E. Borcherds, Automorphic forms with singularities on Grassmannians, Invent. Math. 132 (1998) 491 [alg-geom/9609022] [INSPIRE].

[109] S. Cecotti, P. Fendley, K.A. Intriligator and C. Vafa, A new supersymmetric index, Nucl. Phys. B 386 (1992) 405 [hep-th/9204102] [INSPIRE].

[110] J.A. Harvey and G.W. Moore, Algebras, BPS states and strings, Nucl. Phys. B 463 (1996) 315 [hep-th/9510182] [INSPIRE].

[111] T. Eguchi, H. Ooguri and Y. Tachikawa, Notes on the K3 Surface and the Mathieu group $M_{24}$, Exper. Math. 20 (2011) 91 [arXiv:1004.0956] [INSPIRE].

[112] M.C.N. Cheng, X. Dong, J. Duncan, J. Harvey, S. Kachru and T. Wrase, Mathieu Moonshine and $N=2$ String Compactifications, JHEP 09 (2013) 030 [arXiv:1306.4981] [INSPIRE].

[113] A. Klemm, M. Kreuzer, E. Riegler and E. Scheidegger, Topological string amplitudes, complete intersection Calabi-Yau spaces and threshold corrections, JHEP 05 (2005) 023 [hep-th/0410018] [INSPIRE].

[114] S.-T. Yau and E. Zaslow, BPS states, string duality and nodal curves on K3, Nucl. Phys. B 471 (1996) 503 [hep-th/9512121] [inSPIRE].

[115] S.H. Katz, A. Klemm and C. Vafa, M theory, topological strings and spinning black holes, Adv. Theor. Math. Phys. 3 (1999) 1445 [hep-th/9910181] [INSPIRE].

[116] A. Banlaki, A. Chowdhury, A. Kidambi and M. Schimpf, On Mathieu moonshine and Gromov-Witten invariants, arXiv: 1811.11619 [INSPIRE].

[117] M.C.N. Cheng, K3 Surfaces, $N=4$ Dyons and the Mathieu Group M24, Commun. Num. Theor. Phys. 4 (2010) 623 [arXiv: 1005.5415] [INSPIRE].

[118] P.S. Aspinwall and J. Louis, On the ubiquity of K3 fibrations in string duality, Phys. Lett. B 369 (1996) 233 [hep-th/9510234] [INSPIRE].

[119] S.A. Cherkis and J.H. Schwarz, Wrapping the M-theory five-brane on K3, Phys. Lett. B 403 (1997) 225 [hep-th/9703062] [INSPIRE].

[120] D.R. Morrison and C. Vafa, Compactifications of F-theory on Calabi-Yau threefolds. 1, Nucl. Phys. B 473 (1996) 74 [hep-th/9602114] [INSPIRE].

[121] M. Kreuzer and H. Skarke, PALP: A package for analyzing lattice polytopes with applications to toric geometry, Comput. Phys. Commun. 157 (2004) 87 [math/0204356] [INSPIRE].

[122] Z. Duan, J. Gu and A.-K. Kashani-Poor, Computing the elliptic genus of higher rank E-strings from genus O GW invariants, JHEP 03 (2019) 078 [arXiv:1810.01280] [INSPIRE].

[123] J. Gu, B. Haghighat, K. Sun and X. Wang, Blowup Equations for 6d SCFTs. I, JHEP 03 (2019) 002 [arXiv: 1811.02577] [inSPIRE].

[124] J. Gu, A. Klemm, K. Sun and X. Wang, Elliptic Blowup Equations for 6d SCFTs. II: Exceptional Cases, arXiv:1905.00864 [INSPIRE]. 
[125] D.R. Morrison and W. Taylor, Classifying bases for 6D F-theory models, Central Eur. J. Phys. 10 (2012) 1072 [arXiv:1201.1943] [INSPIRE].

[126] J. Choi, S. Katz and A. Klemm, The refined BPS index from stable pair invariants, Commun. Math. Phys. 328 (2014) 903 [arXiv:1210.4403] [INSPIRE].

[127] R. Gopakumar and C. Vafa, M theory and topological strings. 1., hep-th/9809187 [INSPIRE].

[128] T. Eguchi and K. Sakai, Seiberg-Witten curve for E string theory revisited, Adv. Theor. Math. Phys. 7 (2003) 419 [hep-th/0211213] [INSPIRE].

[129] http://www.th.physik.uni-bonn.de/groups/klemm/data.php.

[130] V.V. Batyrev, Dual polyhedra and mirror symmetry for Calabi-Yau hypersurfaces in toric varieties, J. Alg. Geom. 3 (1994) 493 [alg-geom/9310003] [INSPIRE].

[131] M. Kreuzer and H. Skarke, Complete classification of reflexive polyhedra in four-dimensions, Adv. Theor. Math. Phys. 4 (2002) 1209 [hep-th/0002240] [INSPIRE].

[132] F. Schöller and H. Skarke, All Weight Systems for Calabi-Yau Fourfolds from Reflexive Polyhedra, Commun. Math. Phys. 372 (2019) 657 [arXiv: 1808. 02422] [INSPIRE].

[133] Y.-C. Huang and W. Taylor, On the prevalence of elliptic and genus one fibrations among toric hypersurface Calabi-Yau threefolds, JHEP 03 (2019) 014 [arXiv:1809.05160] [INSPIRE].

[134] V. Braun, Toric Elliptic Fibrations and F-theory Compactifications, JHEP 01 (2013) 016 [arXiv:1110.4883] [INSPIRE].

[135] D.A. Cox and S. Katz, Mirror symmetry and algebraic geometry, AMS, (2000).

[136] P.S. Aspinwall and M.R. Plesser, General Mirror Pairs for Gauged Linear $\sigma$-models, JHEP 11 (2015) 029 [arXiv:1507.00301] [INSPIRE].

[137] S. Hosono, A. Klemm and S. Theisen, Lectures on mirror symmetry, Lect. Notes Phys. 436 (1994) 235 [hep-th/9403096] [INSPIRE].

[138] Y. Kimura, Discrete gauge groups in certain F-theory models in six dimensions, JHEP 07 (2019) 027 [arXiv: 1905.03775] [INSPIRE].

[139] L.B. Anderson, J. Gray and P.-K. Oehlmann, F-Theory on Quotients of Elliptic Calabi-Yau Threefolds, arXiv: 1906.11955 [INSPIRE].

[140] P. Berglund, A. Klemm, P. Mayr and S. Theisen, On type IIB vacua with varying coupling constant, Nucl. Phys. B 558 (1999) 178 [hep-th/9805189] [INSPIRE].

[141] M. Bershadsky, T. Pantev and V. Sadov, F theory with quantized fluxes, Adv. Theor. Math. Phys. 3 (1999) 727 [hep-th/9805056] [INSPIRE].

[142] R. Friedman, J. Morgan and E. Witten, Vector bundles and F-theory, Commun. Math. Phys. 187 (1997) 679 [hep-th/9701162] [INSPIRE].

[143] P. Jefferson, H.-C. Kim, C. Vafa and G. Zafrir, Towards Classification of 5d SCFTs: Single Gauge Node, arXiv:1705.05836 [INSPIRE].

[144] P. Jefferson, S. Katz, H.-C. Kim and C. Vafa, On Geometric Classification of 5d SCFTs, JHEP 04 (2018) 103 [arXiv: 1801.04036] [INSPIRE].

[145] L. Bhardwaj and P. Jefferson, Classifying 5d SCFTs via 6d SCFTs: Rank one, JHEP 07 (2019) 178 [arXiv: 1809.01650] [InSPIRE]. 
[146] L. Bhardwaj and P. Jefferson, Classifying 5d SCFTs via 6d SCFTs: Arbitrary rank, JHEP 10 (2019) 282 [arXiv: 1811.10616] [INSPIRE].

[147] M. Del Zotto, J.J. Heckman and D.R. Morrison, 6D SCFTs and Phases of 5D Theories, JHEP 09 (2017) 147 [arXiv:1703.02981] [INSPIRE].

[148] F. Apruzzi, L. Lin and C. Mayrhofer, Phases of 5d SCFTs from M-/F-theory on Non-Flat Fibrations, JHEP 05 (2019) 187 [arXiv: 1811.12400] [INSPIRE].

[149] F. Apruzzi, C. Lawrie, L. Lin, S. Schäfer-Nameki and Y.-N. Wang, 5d Superconformal Field Theories and Graphs, arXiv:1906.11820 [INSPIRE].

[150] F. Apruzzi, C. Lawrie, L. Lin, S. Schäfer-Nameki and Y.-N. Wang, Fibers add Flavor, Part I: Classification of 5d SCFTs, Flavor Symmetries and BPS States, arXiv:1907.05404 [INSPIRE].

[151] F. Apruzzi, C. Lawrie, L. Lin, S. Schäfer-Nameki and Y.-N. Wang, Fibers add Flavor, Part II: 5d SCFTs, Gauge Theories and Dualities, arXiv:1909.09128 [INSPIRE].

[152] L. Bhardwaj, On the classification of 5d SCFTs, arXiv:1909.09635 [INSPIRE].

[153] L. Bhardwaj, P. Jefferson, H.-C. Kim, H.-C. Tarazi and C. Vafa, Twisted Circle Compactifications of 6d SCFTs, arXiv:1909.11666 [INSPIRE].

[154] J. Gu, A. Klemm, K. Sun and X. Wang, Work in progess.

[155] T. Weigand, F-theory, PoS (TASI2017) 016 [arXiv: 1806.01854] [INSPIRE].

[156] M. Cvetič and L. Lin, TASI Lectures on Abelian and Discrete Symmetries in F-theory, PoS(TASI2017)020 [arXiv: 1809.00012] [INSPIRE].

[157] P.-K. Oehlmann, J. Reuter and T. Schimannek, Mordell-Weil Torsion in the Mirror of Multi-Sections, JHEP 12 (2016) 031 [arXiv:1604.00011] [INSPIRE].

[158] X. De la Ossa, B. Florea and H. Skarke, D-branes on noncompact Calabi-Yau manifolds: $k$-theory and monodromy, Nucl. Phys. B 644 (2002) 170 [hep-th/0104254] [INSPIRE]. 